\title{
The influence of spatial variance on rock strength and mechanism of failure
}

Danqing Gao

West Virginia University, dngao@mix.wvu.edu

Follow this and additional works at: https://researchrepository.wvu.edu/etd

Part of the Mining Engineering Commons

\section{Recommended Citation}

Gao, Danqing, "The influence of spatial variance on rock strength and mechanism of failure" (2020). Graduate Theses, Dissertations, and Problem Reports. 7703.

https://researchrepository.wvu.edu/etd/7703

This Dissertation is protected by copyright and/or related rights. It has been brought to you by the The Research Repository @ WVU with permission from the rights-holder(s). You are free to use this Dissertation in any way that is permitted by the copyright and related rights legislation that applies to your use. For other uses you must obtain permission from the rights-holder(s) directly, unless additional rights are indicated by a Creative Commons license in the record and/ or on the work itself. This Dissertation has been accepted for inclusion in WVU Graduate Theses, Dissertations, and Problem Reports collection by an authorized administrator of The Research Repository @ WVU.

For more information, please contact researchrepository@mail.wvu.edu. 


\title{
The influence of spatial variance on rock strength and mechanism of failure
}

\section{Danqing Gao}

\author{
Dissertation submitted \\ to the \\ Benjamin M. Statler College of Engineering and Mineral Resources \\ West Virginia University \\ in partial fulfillment of the requirements \\ for the degree of \\ Doctor of Philosophy \\ in \\ Mining Engineering
}

\section{Brijes Mishra, Ph.D., Chair \\ Yi Luo, Ph.D. \\ Felicia Peng, Ph.D. \\ Ihsan B Tulu, Ph.D. \\ Ming Gu, Ph.D.}

Department of Mining Engineering

\author{
Morgantown, West Virginia \\ 2020
}

Keywords: Rock, heterogeneity, Extreme Value distribution, spatial variance, correlation, MATLAB, stochastic simulation

Copyright 2020 Danqing Gao 


\section{ABSTRACT \\ The influence of spatial variance on rock strength and mechanism of failure Danqing Gao}

The heterogeneity in the rock formation affects both rock behavior and the strength. The effect of heterogeneity is observed both at the laboratory scale and at the rockmass level. The mechanical properties of intact rock vary considerably at laboratory scale and often an average value is used for design purposes. Similarly, the values are arbitrarily scaled when used at rockmass level. In underground coal mines, the effect of variability of properties is often observed with the erratic roof failure events that occur throughout the mine. The approach often was to use the deterministic values from limited site data to estimate the rock strength and ignoring the inherent variability of rockmass properties. However, current numerical models have successfully captured the global behavior showing the effect of in-situ stress, geology, operational parameters, etc. This dissertation proposes a probabilistic approach that assumes that rockmass properties as random variables and examines its effect on underground coal mine.

The effect of random properties was examined by comparing the deterministic and completely random models which showed the importance of using randomness factor in rocks. Subsequently a spatially correlated random model investigated the influence of rock heterogeneity on rock strength and failure propagation. A random field database with specific spatial correlation was created for each physico-mechanical property using laboratory data and Extreme Value stochastic model in MATLAB. Two scale-measured parameters defined the correlation length, which controls the spatially correlated random data. Then, to verify the importance of the four parameters, friction, cohesion, and correlation length along the horizontal and vertical axes, one hundred and fifty two random sample data are generated. The stress for each specimen is tracked at different loading steps with different spatial correlation factors. This approach determined the effect of material model parameters affect the internal stress distribution for intact rocks. The models were further validated by predicting the behavior of rocks from controlled triaxial tests. Results from the laboratory tests were matched the predicted behavior from numerical models verifying the proposed stochastic method. 
The stochastic method was then implemented in the three-dimensional numerical model to investigate a longwall mine operating in Pittsburgh seam. The influence of random field data on entry roof in the longwall mining system was investigated. Based on Extreme Value stochastic model, the realistic random field database added two scale-measured parameters from both horizontal and vertical directions to control the spatial correlation length. This model also considered a number of cutting sequences, for identifying the effect of the spatial variance on the roof behavior. Finally, the outcome of the dissertation was to use probabilistic approach for demonstrating the heterogeneous characteristic of rock and the influence of spatial variance on the failure mechanism of both intact rock and rockmass.

\section{Keywords}

Rock, heterogeneity, Extreme Value distribution, spatial variance, correlation, stochastic simulation 


\section{ACKNOWLEDGMENTS}

I would like to thank all the people who have contributed to the preparation and completion of this dissertation. Without the support of my advisor, colleagues, friends, and family, the completion of this dissertation would have never been possible.

First, I would like to express my gratitude to my supervisor Dr. Brijes Mishra. He has been more like a mentor than a professor throughout my graduate study. I would like thank him for his invaluable patience, guidance, and assistance during my $\mathrm{PhD}$ at West Virginia University.

I would like to thank my committee members Drs. Yi Luo, Felicia Peng, Ihsan Berk Tulu, and Ming Gu for their support and invaluable suggestions during my research at WVU.

This work was performed under a project sponsored by the National Institute for Occupational Safety and Health (NIOSH). I would thank them for their continued support and funding.

I would like to thank my friends in the Department of Mining Engineering at WVU.

Additionally, I would like thank Karen Centofanti, Genette Chapman, and Karla Vaughan for providing the administrative support throughout my time at WVU.

I want to specially thank my family members: my father Jianzhong Gao, my mother Hongmei $\mathrm{Xu}$, and my thoughtful husband Jian Yang. I thank them for their endless love, constant support, and encouragement. Finally, thanks to my sweet daughter, Tracy Gao Yang, for bringing happiness into my life. 


\section{Table of Contents}

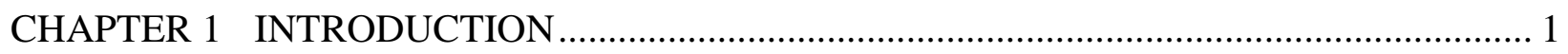

1.1 Variation in mechanical properties of rocks ................................................................ 1

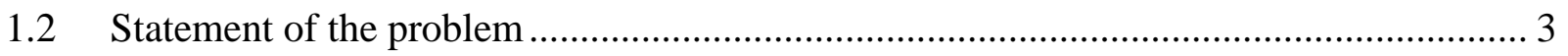

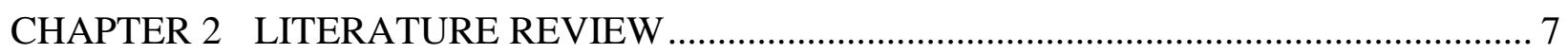

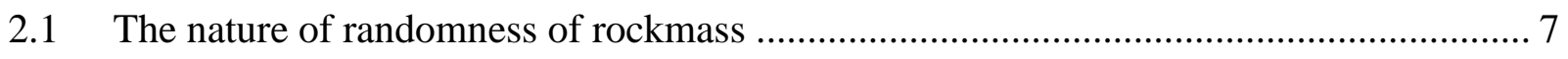

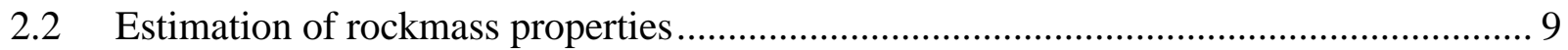

2.3 Application of stochastic method on rock properties .................................................. 12

2.4 The influence of spatial variance on rockmass ........................................................... 16

CHAPTER 3 COMPARISON BETWEEN DETERMINISTIC AND RANDOM MODELS .. 18

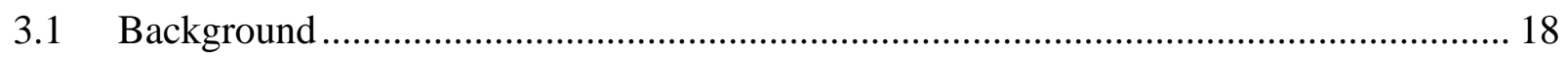

3.2 Laboratory test and statistical analysis ................................................................ 19

3.2.1 Laboratory test and results ........................................................................... 19

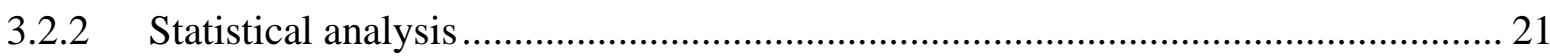

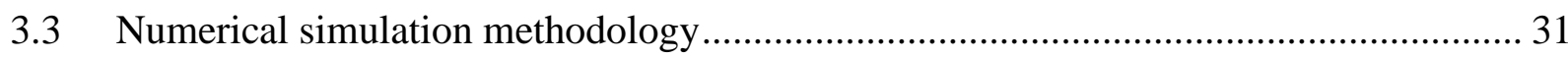

3.3.1 Generation of random mechanical parameters ................................................ 32

3.3.2 Numerical simulation method......................................................................... 33

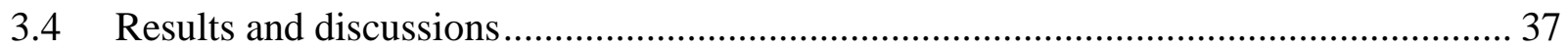

3.4.1 Influence of heterogeneity on rock behavior ..................................................... 37

3.4.2 Sensitivity analysis of element size on the simulation........................................... 40

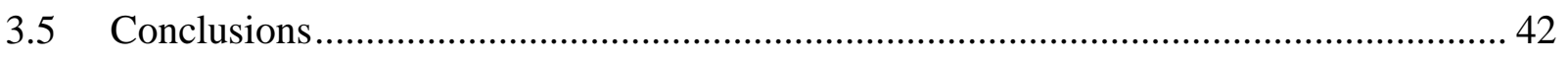

CHAPTER 4 EFFECT OF SPATIAL VARIANCE FACTOR ON RANDOM MODEL .......... 44

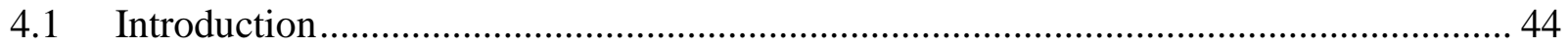

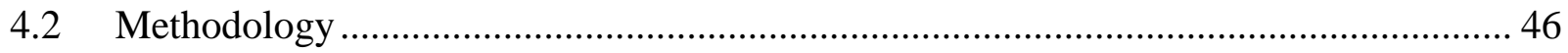

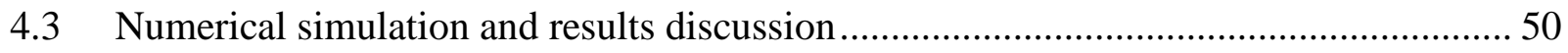

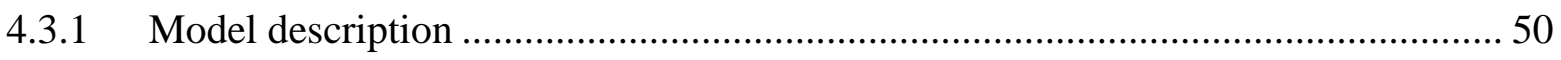

4.3.2 Comparison among traditional deterministic model, completely random model, and random model with spatial correlation .............................................................................. 52

4.3.3 Results discussion for the random model with spatial correlation factor ................ 54

4.4 Sensitivity analysis for four different parameters: friction, cohesion, correlation length along horizontal and vertical axis..................................................................................... 57

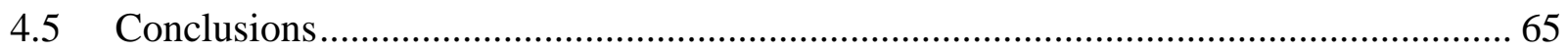




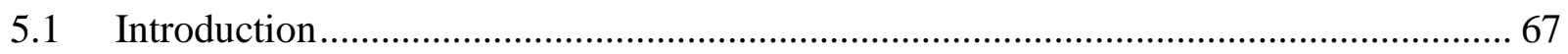

5.2 Comparison between laboratory tests and numerical simulations ................................... 68

5.2.1 Model design for Hoek-cell triaxial laboratory tests ................................................ 68

5.2.2 Model descriptions for numerical simulations..................................................... 73

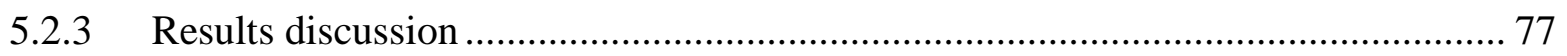

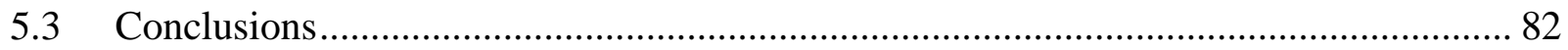

CHAPTER 6 CASE STUDY: PROBABILISTIC EXAMINATION OF SPATIAL VARIANCE EFFECTS ON THREE DIFFERENT FIELD SCALED MODELS ……………...................... 84

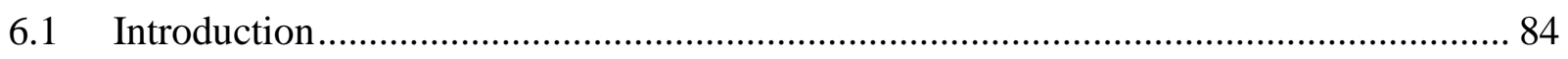

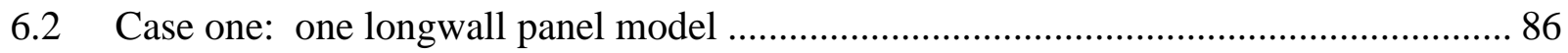

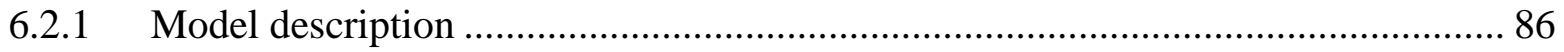

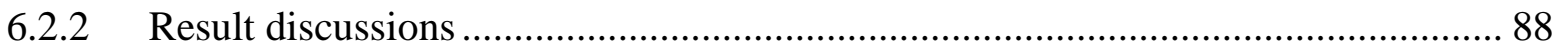

6.3 Case two: Cumberland longwall mine model study .................................................... 90

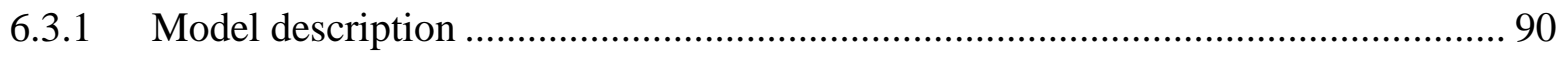

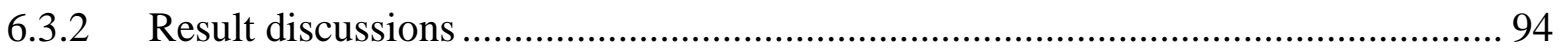

6.4 Case three: Gauss distribution used in one pillar scale model..................................... 103

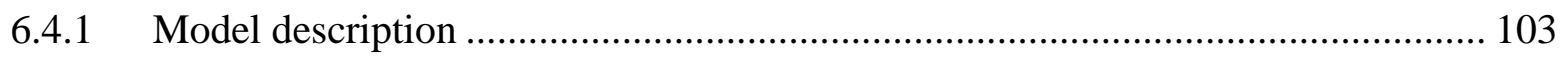

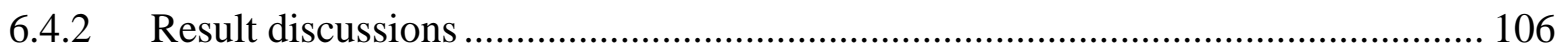

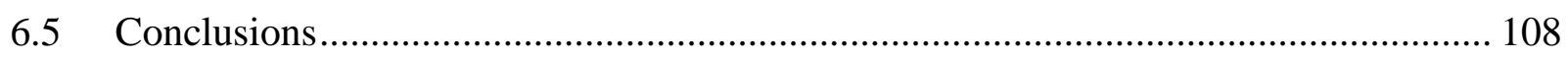

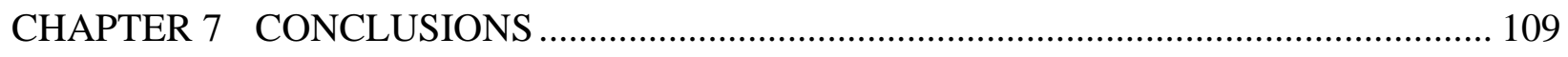

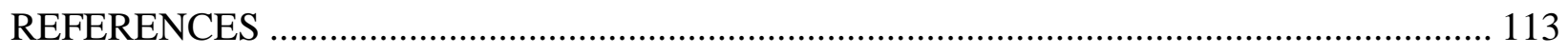

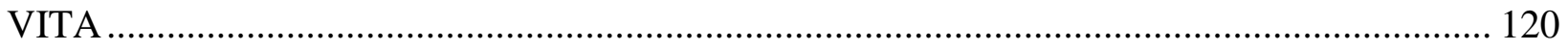




\section{LIST OF TABLES}

Table 3.1 Test results summary of the mechanical properties........................................... 20

Table 3.2 Mean values and standard deviation of the mechanical properties ........................... 20

Table 3.3 Uniaxial Compressive Strength (UCS) for the 1 st group. ....................................... 28

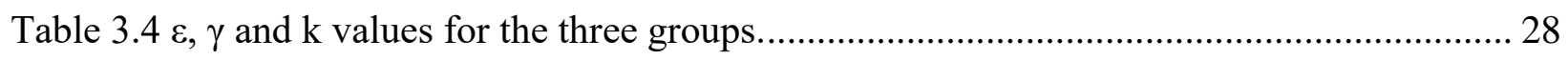

Table 3.5 Probability Density Function (PDF) function for the three groups ........................... 29

Table 3.6 Parameters of mechanical properties determined by Extreme Value distribution........ 31

Table 3.7 Peak strengths of rock specimens for different distributed material properties ......... 34

Table 3.8 Peak strength for 2D and 3D models with same element number conditions............. 39

Table 3.9 Peak strength for 2D and 3D models with different element number conditions........ 41

Table 4.1 Mean values for the material properties used in the rock specimens ....................... 51

Table 4.2 Summary for safety factors of the three different models ...................................... 52

Table 5.1 Summary of center coordinates for each drill hole .............................................. 70

Table 5.2 Summary of minimum principal stress for each specimen under different confining

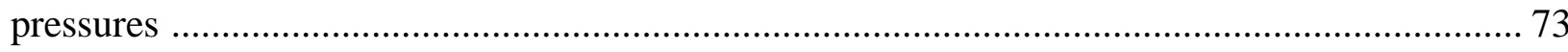

Table 5.3 Summary about friction angle and cohesion values for each specimen .................... 75

Table 5.4 Strength comparison between laboratory test and numerical simulation ................... 82

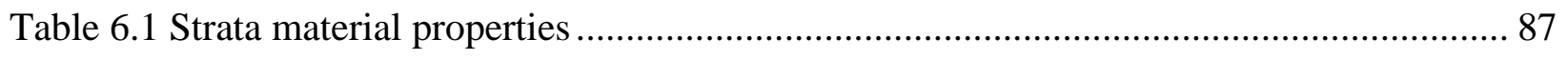

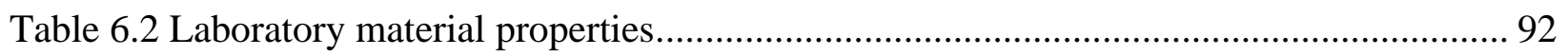

Table 6.3 Random values for the model with spatial variance ............................................. 93

Table 6.4 Geological material properties for the pillar..................................................... 104 


\section{LIST OF FIGURES}

Figure 2.1 Variation of tensile strength of Skin-Komatsu andesite determined by Brazilian method.

Figure 2.2 Crosscut-view of the material distribution of one type of nature rock (the different colors represent different material properties)

Figure 3.1 Comparations for three different parameters between observed and theoretical extremal distributions 24

Figure 3.2 Probability density function curves for different indices .................................... 26

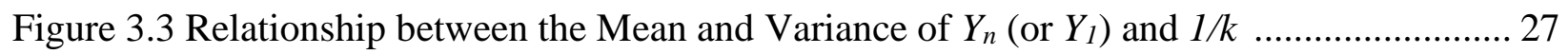

Figure 3.4 Probability density curves of properties of rock core samples ............................... 30

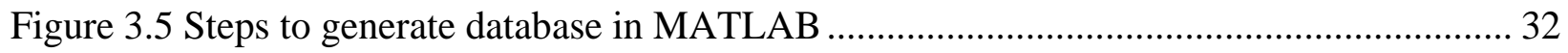

Figure 3.6 Bulk Modulus database generation and filtration in MATLAB ............................. 33

Figure 3.7 Dimensional sketch for the numerical models ................................................ 34

Figure 3.8 Peak strengths of rock specimens for different distributed material properties ......... 35

Figure 3.9 Randomly distributed property schematic diagrams in 2D (cohesion) and 3D (Bulk

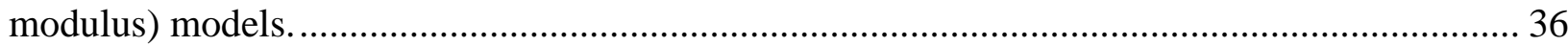

Figure 3.10 Stress-strain curves for 2D and 3D models in same element number conditions ..... 37

Figure 3.11 Plot of element state with different properties ................................................. 39

Figure 3.12 Plot of element state with stochastic properties in a three-dimensional rock specimen

Figure 3.13 Stress-strain curves for 2D and 3D models in different element number conditions 41

Figure 4.1 Existence of spatial variance in different types of rock specimens ......................... 47

Figure 4.2 Representation of one strength variation at different depths .................................. 48

Figure 4.3 Steps to generate the spatial correlated database in MATLAB .............................. 49

Figure 4.4 The geometry of FLAC8.0 model …........................................................... 51

Figure 4.5 Material distributions for three representative models ........................................ 51

Figure 4.6 Histogram for the safety factor of the three different models ................................ 53

Figure 4.7 Stress-strain curves for the three representative different models........................... 54

Figure 4.8 Vertical stress variations along the central axes of the specimens ........................... 56 
Figure 4.9 Yield state for rock specimen with different spatial dependence 57

Figure 4.10 Friction, cohesion contours vs. yield state contours with different loading steps ..... 60

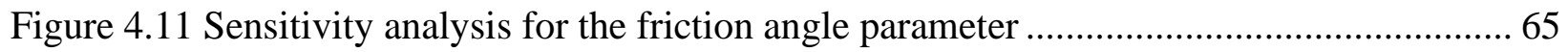

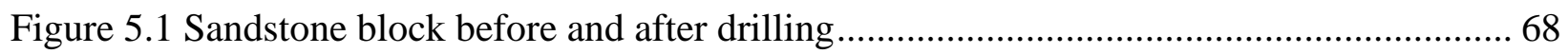

Figure 5.2 Schematic diagram for the Hoek-cell Triaxial experimental equipment................... 72

Figure 5.3 Schematic diagram of the large rock block created in FLAC8.0 ........................... 74

Figure 5.4 Random field's distributions for two parameters: cohesion and friction angle.......... 76

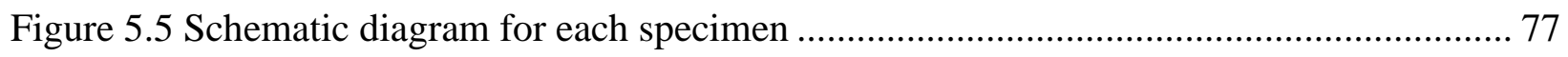

Figure 5.6 Failure pattern for each specimen under 200psi confining pressure ....................... 79

Figure 5.7 Failure pattern for each specimen under 200psi confining pressure ....................... 80

Figure 5.8 Strength comparison between laboratory test and numerical simulation .................. 81

Figure 6.1 Geometry for the longwall panel model ......................................................... 86

Figure 6.2 Boundary condition for the longwall panel model ............................................. 87

Figure 6.3 Comparison of vertical stress between FLAC3D and LAMODEL........................... 88

Figure 6.4 Schematic diagram for quarter part of one longwall panel ................................... 89

Figure 6.5 Difference of stress distribution along each pillar between deterministic and stochastic

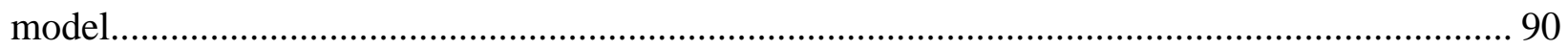

Figure 6.6 Representative lithology used in the FLAC3D model ....................................... 91

Figure 6.7 Random field's distributions for the two parameters ......................................... 94

Figure 6.8 Vertical stress contour for the global model ...................................................... 95

Figure 6.9 Vertical stress contours for the immediate roof section ....................................... 96

Figure 6.10 Minimum principal stress contours for the immediate roof section ..................... 98

Figure 6.11 Failure state contours for the immediate roof section. (Five excavations)............. 102

Figure 6.12 Mine layout for the quarter pillar ................................................................. 104

Figure 6.13 Quarter pillar with stochastic material definition ........................................... 106

Figure 6.14 Result contours for the quarter pillar............................................................. 107 


\section{LIST OF ABBREVIATIONS AND SYMBOLS}

\section{List of Symbols}

$$
\begin{aligned}
a & =\text { the speed at which the spatial dependence decreases along one principal axis } \\
b & =\text { the speed at which the spatial dependence decreases along another principal axis } \\
E_{M} & =\text { the in-situ deformation modulus of rockmass } \\
E_{\text {max }} & =\text { upper bound on the deformation modulus } \\
E_{\text {mean }} & =\text { mean value of the in-situ deformation modulus } \\
E_{\text {min }} & =\text { lower bound on the deformation modulus } \\
F_{Y}^{-1} & =\text { inverse cumulative distribution function of one statistic distribution } \\
k & =\text { shape parameter of Extreme Value distribution } \\
P & =\text { Cumulative distribution function } \\
Q & =\text { value of } Q \text { in the Q-system } \\
t & =\text { a scalar parameter including both the spacing value and orientation } \\
t_{1}, t_{2} & =\text { spatial interval values along the field's major and minor axes respectively } \\
u & =\text { one specific location in the real field } \\
\alpha & =\text { shape parameter of Weibull distribution } \\
\beta & =\text { scale parameter of Weibull distribution } \\
\gamma(t) & =\text { semi-variogram } \\
\varepsilon & =\text { lower bound of Extreme Value distribution } \\
\mu_{l n Y} & =\text { mean value of the natural log of the parameter } Y \\
\mu_{X} & =\text { mean value of } X \\
v & =\text { scale parameter of Extreme Value distribution } \\
\sigma_{c i} & =\text { uniaxial compressive strength } \\
\sigma_{l n Y} & =\text { standard deviation of the natural log of the parameter } Y \\
\sigma_{\mu} & =\text { smallest strength of Weibull distribution } \\
\Phi(z) & =\text { cumulative distribution function of the standard correlated Gaussian distribution }
\end{aligned}
$$




\section{List of Abbreviations}

GSI = geological strength index

NIOSH $=$ National Institute for Occupational Safety and Health

$\mathrm{RMR}=$ rockmass rating

UCS = uniaxial compressive strength 


\section{CHAPTER 1 INTRODUCTION}

\subsection{Variation in mechanical properties of rocks}

In underground coal mines roof failure and rib deformation often contribute to numerous injuries and fatalities. The size and the scale of mining operations have increased tremendously and therefore the erratic nature of rock failure in both roof and pillar have followed similar behavior. For instance, in the Pittsburgh coal seam, roof control issues are quite common due to the presence of laminated shale. This particular rock type fails uniquely under a certain stress orientation that has been well documented and implemented in mine design. Using in-situ measurements, empirical models and calibrated numerical models a reliable methodology is commonly practiced in designing underground mines. However, the occurrence of roof, rib failures and the erratic nature of these roof falls seems to suggest that all factors are not always considered during mine modeling. One such parameter is the variability of the rock both at intact or laboratory scale level and rock mass level.

Rock is heterogeneous by formation, indicating that the constituents at grain level are not in uniform distribution (Brown, 1974). In addition, the rock at large scale level often referred to as "rockmass" has significant level of breakage and separation known as discontinuity. The separation of rock free from any discontinuity is called "Intact Rock". For both intact and rock mass, the strength is affected by heterogeneity of formation. In addition, at rockmass level the presence of discontinuity further reduces the strength. The approaches to bridge the two scales have often been difficult particularly at micro level. 
Traditionally, the parameters such as the physico-mechanical behavior and strength and insitu stresses by have been estimated by using mean values from a limited number of samples from laboratory and field tests. For the last five decades, rock stability analysis was conducted using analytical and empirical solutions formulation and in the last two decades numerical simulation are used extensively (Bieniawski, 1968, Recio-Gordo, 2012 and Soltani, 2015). The deterministic approach is regularly used in mine design analysis.

With a deterministic approach, the averaged values from the laboratory tests or site data are used for estimating the rock parameters for the design or safety assessment. The inherent variation in rock properties is often excluded from mine stability analysis which increases the potential for inaccurate estimation of rock strength. For instance, in pillar design the safety factors are commonly used for a quantitative assessment of state of pillars. They are calculated by using strength of the rock over the stress developed due to the design. When the rock strength is overestimated, larger pillars are designed which reduces the stability of the pillars based on the apparent strength causing the safety factor to be lower than a threshold value. (Deng, 2003). Underestimation of the strength will produce larger mine pillars making the mining operation uneconomical (Jade, 2003). Therefore, estimating accurate strength is the key for an optimized pillar design. However, the lack of widely accepted probabilistic methods that considered the variability of rockmass properties have restricted the application and development of stochastic methods (Fang, 2002; Kim \& Gao, 1995; Mingli, 2000). Using stochastic/random approach may provide an accurate estimation of strength.

The stochastic/random approach provides a unique method to include various ranges of properties from intact rock and then scale it to the behavior of rock mass. Numerical simulation coupled with stochastic properties provides an alternative approach to investigate the failure 
process of rock materials. Traditionally, stochastic finite element method was extensively used for slope stability analysis. The focus has shifted to displacement discontinuity and finite difference methods. In addition, development of distinct element method has increased the research capabilities in the field of rock mechanics. Numerical methods also provide initial stress, strain fields, or the final stress state of a rock structure. With the improvement in the computing environment and practical demands in rock mechanics, rock failure analysis has changed from partial simulation to the entire structure.

This research developed and showed application of stochastic analysis in mine design using combination of finite difference method and elastic damage mechanics.

\subsection{Statement of the problem}

Whether from a macroscopic or microscopic view, underground rockmass shows large inherent variation in its physico-mechanical properties. Including the thickness of the strata, the moisture content, the porosity, and the history of the depositions, all these factors influence the mechanical responses and failure behaviors of the underground rockmass significantly.

Pillar stability is critical for mining operations. Room-and-pillar and in the gateroad section of the longwall mines pillars are the primary support for the overburden. Unstable pillars can lead to massive collapse of the panels, gate blockage, pillar bump, and subsidence (Mark, 1987). When coal pillars fail, the approach is to recalibrate the input properties of the model until the failure predicted in the model is in agreement with the ground condition. A drawback of this approach is the usage of deterministic values to represent the strength of the pillars, ignoring the inherent variability that exists in the properties of coal and the surrounding rockmass (Amusin, 1979; Barton, 1974; Bieniawski, 1993; Brady and Brown, 1993). In order to analyze the 
rockmass behavior, one must assess the inherent variability of rockmass mechanical properties, such as the strength and deformation properties for the entire mine.

The main goal of this research is to suggest a realistic approach to simulate the inherent spatial variability of rockmass. This approach accounts for the uncertainties due to both the variability of the rock material properties and the spatial variance on rockmass. When describing the rockmass properties, friction and cohesion are the main factors and are considered random variables.

This research aims to use the proposed probabilistic method for demonstrating the heterogeneous characteristic of rock from the perspective of material properties, observing the influences of spatial variance on failure mechanism for both rock specimen in laboratory and rockmass in the real site. This dissertation aims to understand these behaviors through the following steps:

First, a comparative analysis will be conducted between the deterministic and completely random models. The main goal of this step will be to show the importance of considering the randomness factor of rock.

Second, this research will consider one spatial correlated random model to investigate the influence of rock heterogeneity on the rock strength and failure propagation. The random field database will be created with specific spatial correlation for each physical-mechanical property through laboratory data and Extreme Value stochastic model in MATLAB. This database will be added to the two scale-measured parameters to define the correlation length, which controls the spatially correlated random data. 
Third, to verify the importance of friction, cohesion, and correlation length along the horizontal and vertical axis respectively, this research will generate 152 random sample data. The stress will be tracked for each specimen at different loading steps and different locations. The main aim of this process will be to investigate the inherent material parameters affecting the internal stress for intact rocks.

Fourth, in order to have confidence in the model extensive triaxial laboratory will be conducted. The real spatial variance distribution will be established through the experiments. Based on the mechanical parameters obtained from the laboratory, the proposed method will reproduce the material distribution of the intact rock block. If the results matched with each other, the proposed stochastic method would be appropriate.

Finally, this research will conduct a three-dimensional longwall mine model analysis using the method proposed. The influence of the random field data on entry roofs in the longwall mine system is expected. This research will create realistic random field database based on the Extreme Value stochastic model, adding two scaler-measured parameters from both the horizontal and vertical directions to control the spatial correlation length. This model will also use few cutting sequences to observe if the spatial variance has any significant influence on the roof behavior.

The proposed approach mentioned above assists in characterizing the nature of inherent variability of the mechanical properties of rockmass. It provides a method for assessing the variability of the rockmass properties, which cannot be obtained through in-situ measurements. These simulated rockmass characteristics could help rock engineers to achieve a more rational design of underground structures, compared to traditional design methods based on deterministic approaches. 
Many fundamental principles and mechanical phenomena are repeated by using FLAC; they then made comparisons of the numerical results with experimental results in order to validate the numerical model and analyze the intrinsic mechanism in rock mechanics and engineering throughout this dissertation.

This research assigned mechanical properties throughout the specimens by following certain distribution functions, regarding heterogeneity in rocks to reflect the nonlinearity during rock failure process. Even through adopting a simple constitutive law in the numerical model, we can obtain various and complex macroscopic results. 


\section{CHAPTER 2 LITERATURE REVIEW}

Rock, as a natural material, is discontinuous, inhomogeneous, anisotropic, and not elastic; this means that rock failure can manifest in many ways. There are many factors that influence the properties of rock, which includes anisotropy, cracks and fissures, pore pressure, rate of loading, time effects, and specimen size and shape (Bieniawski, 1968). The numerical simulation methods used for rock engineering design usually assume that the mean values of rockmass properties, measured or estimated, represent rockmass characteristics. Because of the random distributions of rockmass properties, this assumption can cause misrepresentative results.

Given the inelastic nature of the in-situ rock together with its discontinuous, inhomogeneous, anisotropic and three-dimensional nature, it is not possible to analytically examine and evaluate the complete mechanical behavior of the rockmass, except as an approximate approach. The problem becomes even more intractable if gas or fluid is involved, as in coal and gas outbursts, hydraulic fracturing, etc. Various methods have been developed to estimate rockmass properties.

\subsection{The nature of randomness of rockmass}

Rockmass is an inhomogeneous and discontinuous medium. The determination of the mechanical properties of rockmass always involves a number of uncertainties. This is due to the variability of the rock material and the fracture patterns in rockmass. The results of the laboratory and in-situ tests are thus affected by both the chosen test locations and volumes of rocks involved in the tests. Rock engineers frequently resort to a simplifying assumption that the rockmass is a homogeneous and isotropic medium for designing rock structures. Rock is a naturally formed material, which has undergone geologic processes. Even a seemingly 
homogeneous rockmass lacks consistency in its internal state. Rockmass, on an engineering scale, is a complicated natural composite material found in-situ consisting of rock material and structural discontinuities. Rock material is the solid part of the rockmass typically obtained in an unbroken drill core, usually called intact rock. Structural discontinuities include joints, bedding planes, partings, and shear zones. The use of this definition tends to imply something larger, less individualized and less distinct from its surroundings. Rockmass properties are complex functions of the properties of intact rock and discontinuities in rockmass.

Rock engineers frequently encounter difficulties when handling the inherent variability of rockmass properties from the initial stage of site characterization to the final design. The computational methods used for rock engineering design typically assume that the rockmass is a homogeneous and isotropic medium. Then, the final design is adjusted according to the historical data by so-called expert judgements. These empirical formulas are developed from laboratory and field experiments. There are various types of uncertainties involved in the determination of rockmass properties and in-situ stresses. Therefore, design engineers have attempted to cope with these uncertainties by using safety factors. Even when using a very conservative safety factor in the design, failures of rock structures still occur. Therefore, the assessment of the variability of rockmass properties must be performed and incorporated in the design itself.

The variability of mechanical properties of rockmass, such as the strength and the deformability, must be quantified and incorporated in design. Due to the complicated and lengthy geologic process, rock has become an extremely complicated medium and various uncertainties are involved in the determination of mechanical property parameters. Therefore, the inherent variability of mechanical properties of rocks must been characterized by employing the 
concept of random variable. The engineering design can be performed realistically only if the variation of the mechanical properties of rocks is also incorporated realistically.

\subsection{Estimation of rockmass properties}

In order to supplement analytical modelling and to incorporate some of the rockmass idiosyncrasies, albeit indirectly, rockmass classification systems have been developed mainly to support rock-engineering design, the most well-known ones being the Rockmass Rating (RMR), the Quality $(Q)$, and the Geological Strength Index $(G S I)$. These systems are most useful for rock engineering, especially for estimating rock support.

Bieniawski provided a useful method for estimating in-situ deformability of rockmass based on the geomechanics classification of rockmass (the RMR system) (Bieniawski, 1978):

$$
E_{M}=2 R M R-100
$$

where $E_{M}$ is the in-situ deformation modulus of rockmass (GPa); $R M R$ is the rockmass rating (for $R M R>50)$.

The above correlation is valid only for $\mathrm{RMR}>50$. Therefore, the value of $E_{M}$ ranges from 0 to $100 \mathrm{GPa}$ as a result of the above correlation.

The $Q$-system of rockmass classification was developed for assessing tunnel stability (Barton, 1974). Barton supplemented the data of Bieniawski with his own results by the $Q$-system and found the following approximations (Bieniawski, 1978):

$$
\begin{gathered}
E_{\text {mean }}=25 \log Q \\
E_{\text {mean }}=40 \log Q \\
E_{\text {mean }}=10 \log Q
\end{gathered}
$$

where 
$Q$ is the value of $Q$ in the $Q$-system;

$E_{\text {mean }}$ is the mean value of the in-situ deformation modulus (GPa);

$E_{\min }$ is the lower bound on the deformation modulus (GPa);

$E_{\max }$ is the upper bound on the deformation modulus (GPa).

Since the value of $Q$ varies from 0.001 to 1000 , the value of $E_{\text {mean }}$ ranges from 0 to $75 \mathrm{GPa}$. This approach is comparatively the same as Bieniawski's, especially when using the following relationship:

$$
R M R=9 \ln Q+44
$$

The Geological Strength Index $(G S I)$ provides a system for estimating the reduction in rockmass strength for different geological conditions as identified by field observations (Hoek, 1994; Hoek \& Kaiser, 1995; Hoek \& Brown, 1997). The GSI system concentrates on the description of two factors, rock structure and block surface conditions. The guidelines given by the GSI system are for the estimation of the peak strength parameters of jointed rockmass. Using the $G S I$ system, provided the $U C S$ value is known, the rockmass deformation modulus $E_{m}$ for $\sigma_{c i}$ $<100 \mathrm{MPa}$ is estimated in GPa from the following equation:

$$
E_{M}=\sqrt{\frac{\sigma_{c i}}{100}} * 10^{\left(\frac{G S I-10}{40}\right)}
$$

where $E_{M}$ is the rockmass deformation modulus; $\sigma_{c i}$ is the uniaxial compressive strength; ans $G S I$ is the Geological Strength Index.

There are many empirical formulas in China for estimating rockmass properties for different rock types (Yu, 1983; Gao \& Shen, 1987; Gu, 1979). One approach, for example, is to consider 
half of the Young's modulus of intact rock obtained from laboratory core test as the deformation modulus of rockmass.

In the previous work, researchers developed these empirical formulas from extensive laboratory and field tests in coal mines; however, even though they validated and updated these formulas using field data, they are still localized and do not reveal the inherent variability. Some physical explanation of these relationships is necessary. Empirical formulas were developed in the former Soviet Union for metamorphic chlorite schists and gneiss crystalline schists (Gureev \&Voronkov, 1974) respectively:

$$
\begin{aligned}
& E_{m}=7870 K_{c p}^{-0.475} \\
& E_{m}=8550 K_{c p}^{-0.725}
\end{aligned}
$$

where $E_{m}$ is the deformation modulus of rockmass in $\mathrm{MN} / \mathrm{m}^{2} ; K_{c p}$ is the area crack porosity coefficient $(\%)$, numerically equal to the area of joints per $\mathrm{m}^{2}$.

However, the determination of each parameter above still involves uncertainties. Experiments developed these empirical formulas; however, they do not disclose the inherent variability of rockmass properties, and the many uncertainties involved in the determination of rockmass properties. Design engineers have been attempting to cope with these uncertainties by using safety factors. Even when a very conservative safety factor is used in design, failures of rock structures still occurs. As such, systematic approaches to assessing the variability of rockmass properties and incorporation of the variability in the design process have gained more attention in rock engineering. 


\subsection{Application of stochastic method on rock properties}

The rockmass is considered a random medium. The overall characteristics of the rockmass depend upon the random nature of intact rocks and the random nature of discontinuities in rockmass. All parameters describing rockmass properties can be considered random variables due to the random nature of the rockmass and the random combination of intact rocks and rock discontinuities. These random variables must be defined through their probability functions based on the results of experimental evidence.

Weibull developed a statistical model to explain the inherent variability of the strength of materials (Weibull. 1939, 1952). He idealized a test sample under tension as an assemblage of elements with statistically independent element properties. He finally concluded that the tensile strength of material follows a probability distribution, which was later termed by his name.

$$
F(x)=1-e^{-\left(\frac{\left(x-\sigma_{\mu}\right)}{\beta}\right)^{\alpha}}
$$

where $\alpha$ is the shape parameter of the distribution; $\sigma_{\mu}$ is the smallest strength; $\beta$ is the scale parameter of the distribution.

This is the well-known Weibull distribution, which shows how the distribution of the breaking strength of materials could be represented by a statistical distribution. This distribution is widely applicable in many fields, such as reliability, fatigue, and fracture mechanics. As such, more attention has been focused on probabilistic considerations for assessing the characteristics of rockmass. 


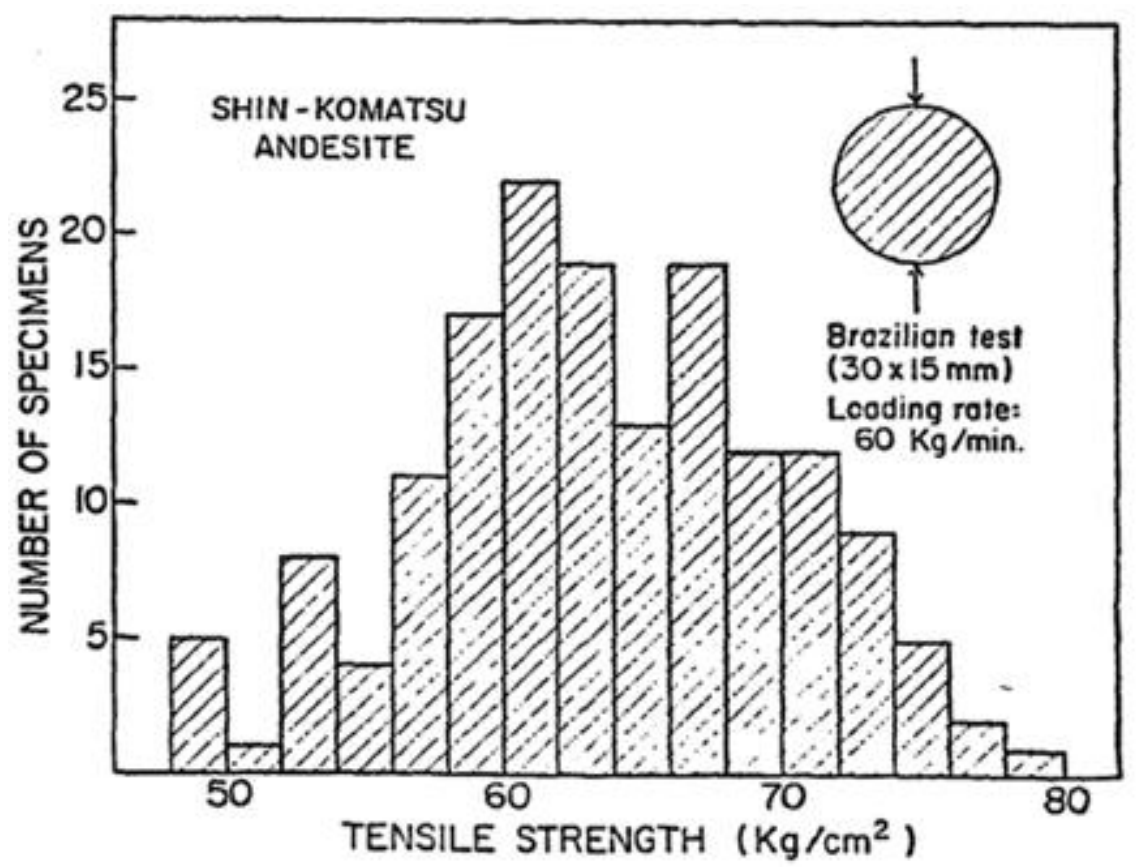

Figure 2.1 Variation of tensile strength of Skin-Komatsu andesite determined by Brazilian method

(Yamaguchi, 1970)

Figure 2.1 shows the failure of a tested sample, caused by a severe flaw in the sample as indicated by Weibull theory. The variation in the tensile strength can be explained as a direct consequence of the probabilistic nature of strength. The most severe flaw in each sample varies in weakening effect. As a result, specimens will show different strengths because they contain extreme flaws of different intensities. This explains the variation that occurs when repeating the same test many times. The variation of the tensile strength reveals the inherent variability of the weakest link in each sample, which is a natural phenomenon in nature. Therefore, the most meaningful task of rock engineers is to develop a probabilistic rock engineering design methodology. A piece of rock is a statistical ensemble of a very large quantity of primary elements; each element is to some degree responsible for the strength of the whole rock. When taking rock samples to test in the laboratory, one is sampling randomly from a large domain of 
rocks. The weakest link governs the overall strength of each sample. Since the mechanical properties of the primary elements are random, the property of the whole sample possesses certain random characteristics. A rock engineering design essentially relies on the characterization of mechanical properties of rockmass.

Similarly, on analysis of the distribution patterns of joints in rockmass it was found that the joints follow a Poisson's process when a line-scan is performed (Priest \& Hudson, 1981; Einstein, 1983; La Pointe, 1985). Therefore, joint spacing follow an exponential distribution:

$$
F(x)=1-e^{-\lambda x}
$$

where $\lambda$ is the intensity parameter of the distribution.

This observation was a great contribution to rock engineering for describing joints in rockmass statistically. In the real field, joints in rockmass are extremely difficult to characterize due to their complicated spatial distribution and large quantity in number. This model provides a practical statistical way to describe joints, revealing the inherent variability of joint distribution.

The mechanical property data obtained from the laboratory core tests are also analyzed by using extreme value statistics (Gumbel, 1958) and concluded that the mechanical properties of intact rocks follow the third type asymptotic distribution of the smallest values (Yegulalp, 1968,1994; Yegulalp \& Mahtab, 1983).

$$
F(x)=1-e^{-\left(\frac{x-\varepsilon}{v-\varepsilon}\right)^{k}}
$$

where $k$ is the shape parameter of the distribution; $\varepsilon$ is the lower bound on $X$, and $v$ is the scale parameter of the distribution.

The corresponding probability density function (PDF) is 


$$
f(x)=\frac{k}{\gamma-\varepsilon}\left(\frac{x-\varepsilon}{\gamma-\varepsilon}\right)^{k-1} e^{-\left(\frac{x-\varepsilon}{\gamma-\varepsilon}\right)^{k}}, x \geq \varepsilon
$$

It is notable that the Weibull distribution is a special case of this general form if expressing the term $(v-\varepsilon)$ by one parameter. The limit boundary is zero for the Weibull distribution; this boundary is called the weakest link in Weibull's theory. However, each real site data has its own lower threshold, and zero is not the corresponding threshold value in most cases. Unlike the Weibull distribution, the Type III Extreme Value distribution is derived under most general conditions, regardless of intended specific field of application.

The concept of extreme values, to which the weakest-link model belongs, first appeared in Chaplin's paper, which related the size effect to extremes (Chaplin, 1880). Griffith proposed a "weakest-link" or "largest flaw" theory, according to which the greatly reduced strengths of isotropic materials were the results of discontinuities, flaws, or defects spread at random throughout the material (Griffith, 1921). Griffith's theory had a profound effect on the thinking of scientists and engineers in the study of material strength. Shortly after the appearance of Griffith's theory, the beginning of the asymptotic behavior of sample extremes are been investigated.

Other investigators have reported the applications of probabilistic methods to problems ranging from foundation design and damage mechanics problems to the assessment of the hydraulic conductivities in situ stresses (Beacher \& Ingra, 1981; Bazant, 1990; Zhang, 1990; Bagtzoglou \& Baca, 1993). 


\subsection{The influence of spatial variance on rockmass}

Random variables and deterministic variables are easily expressed in terms of mean and variance. The variable that is neither random nor deterministic will have a mean and a variance as well. Closely spaced points will have a correlation that is dependent, and the variance will be low (Matheron, 1971). Widely spaced points tend to be independent or random in $\mathrm{Z}$ value and will fall within the range predicted by the variance (Ledvina, 1991).

Due to the long-term complex depositional process, the physical-mechanical properties of rockmass are neither deterministic nor completely random. They tend to be independent at outlying distances and correlated at relatively small distances (Ledvina, 1991). The mechanical behaviors of grain scaled material have some spatial continuities and correlations. The crosscutview plot shown in Figure 2.2 is an example of this correlation. The local spatial continuities exist inherently within the rock, which creates the physico-material properties in the adjacent area to be similar and spatially correlated; however, the material properties are different for those rocks that are located at some distance.

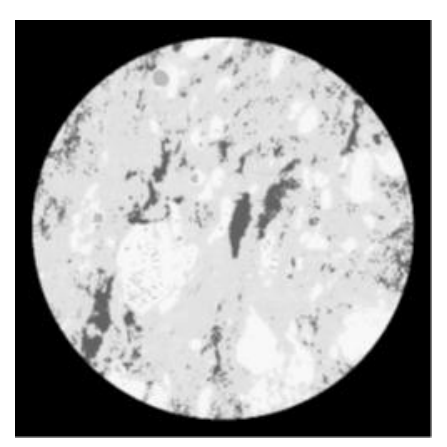

Figure 2.2 Crosscut-view of the material distribution of one type of nature rock (the different colors represent different material properties) (Hubler, 2017)

This phenomenon is spatial variation, defined as the changes that occur in a parameter when measured over distance and direction. This variation is divided into two categories: systematic 
and random. Systematic variation in natural rocks is denoted by either gradual or abrupt changes in rock properties and is caused by the rock forming factors of relief, climate, organisms, time, and parent material. Random variation is not related to any known cause, occurs simultaneously with systematic variation, and may include unrecognized systematic variation (Wilding \& Drees, 1978). Processes that are neither deterministic nor random create these geologic materials (and thus their properties). The concept of the regionalized variable applies well to such variables.

The theory of regionalized variables can estimate unknown values at points between known values, a very useful feature for geologic data at points separated by un-sampled and unknown areas. Such interpolation between knowns is the goal of geo-statistics. A secondary goal is the determination of the accuracy of these estimates.

The proximity is only quantifiably useful if the variable is continuous across the space separating two points. The concept of continuity as applied to a geologic entity, such as a tabular rock unit, implies that the rock layer is mechanically continuous or unbroken over an area (Ledvina, 1991). 


\section{CHAPTER 3 \\ COMPARISON BETWEEN DETERMINISTIC AND RANDOM MODELS}

\subsection{Background}

Heterogeneity affects the physico-mechanical behavior of rock. The inherent variability of rock is one of the major sources of uncertainty that is commonly observed in the strength variation from intact rock to rockmass. Currently, the deterministic approach in solving rock mechanics problems have been widely used, using single mean values of limited site data to estimate rockmass parameters such as strength, deformation modulus, and in-situ stresses (Amusin, 1979; Bieniawski, 1978; Hoek \& Brown, 1978). However, the estimations are always questionable, as the deterministic methods ignore the inherent variation in rockmass.

In order to deal with the difficulties of handling this inherent variability, researchers have been developing various methods to estimate rockmass properties (Fang, 2002; Kim, 1995; Mingli, 2000). With the deterministic method, the deterministic values from laboratory test results or site data are used in order to estimate rock parameters, such as strength and deformation modulus, for the design or safety assessment; the authors pre-define the mechanical parameters and the property distribution used in the numerical simulations, instead of determining them through laboratory tests. These studies usually ignore deviation in the parameters; therefore, they also ignore the inherent variation in rock properties, and as a result, they can overestimate or underestimate the rock strength. Overestimation of coal strength could lead to designing pillars that are smaller than needed, inducing further safety concerns (Deng, 2003). Underestimation of the coal strength could result in too conservative a pillar design, wasting coal (Mark, 1987; Jade, 2003). These variables demonstrate the necessity of using a 
stochastic method to investigate the stability of coal pillars and other rock mechanic problems. The results of a stochastic numerical simulation with mechanical parameters and property distribution determined from laboratory tests should be more realistic.

The research in this chapter proposed a stochastic approach using the Extreme Value distribution for simulating laboratory tests and investigating the influence of rock heterogeneity on the behavior and strength of rock specimens. As the first step, laboratory compression tests were conducted on sandstone specimens to determine the mechanical parameters, followed by a statistical analysis. The results demonstrate that the Extreme Value distribution fits the parameters better than Weibull distribution. Secondly, a stochastic numerical simulation method simulated laboratory tests on rock specimen. Based on the laboratory test results and the Extreme Value distribution, MATLAB was able to generate a random mechanical parameters database. Then, a scripting fish function assigned the random parameters to the elements in $2 \mathrm{D}$ and $3 \mathrm{D}$ FLAC models. Finally, FLAC is used to numerically simulate a series of unconfined compression tests (Itasca, 2017), and the influence of heterogeneity was analyzed on rock behavior and strength.

\subsection{Laboratory test and statistical analysis}

\subsubsection{Laboratory test and results}

This research conducted laboratory compression tests in the Rock Mechanics Laboratory at West Virginia University to study the variation in the mechanical parameters of rocks. In this

study, we prepared and tested eighteen sandstone specimens. The rock core specimens are 4 inches in length and 2 inches in diameter.

Table 3.1 shows the mechanical properties result summary of the eighteen tests: 
Table 3.1 Test results summary of the mechanical properties

\begin{tabular}{|c|c|c|c|c|}
\hline $\begin{array}{c}\text { Test } \\
\text { Number }\end{array}$ & $\begin{array}{c}\text { UCS } \\
\left(\times \mathbf{1 0}^{\mathbf{3}} \mathbf{p s i}\right)\end{array}$ & $\begin{array}{c}\text { Young's } \\
\text { Modulus } \\
\left(\times \mathbf{1 0}^{\mathbf{5}} \mathbf{p s i}\right)\end{array}$ & $\begin{array}{c}\text { Cohesion } \\
\left(\times \mathbf{1 0}^{\mathbf{3}} \mathbf{p s i}\right)\end{array}$ & $\begin{array}{c}\text { Friction Angle } \\
\left({ }^{\circ}\right)\end{array}$ \\
\hline 1 & 11.14 & 25.06 & 2.57 & 40.33 \\
\hline 2 & 16.12 & 38.18 & 2.17 & 42.78 \\
\hline 3 & 9.39 & 11.83 & 2.38 & 39.38 \\
\hline 4 & 8.02 & 11.95 & 1.90 & 43.87 \\
\hline 5 & 5.96 & 9.59 & 1.86 & 46.45 \\
\hline 6 & 8.87 & 15.11 & 4.16 & 46.75 \\
\hline 7 & 9.62 & 19.55 & 1.43 & 47.90 \\
\hline 8 & 10.98 & 14.67 & 3.06 & 44.09 \\
\hline 9 & 7.16 & 13.54 & 1.91 & 50.05 \\
\hline 10 & 7.70 & 15.03 & 2.38 & 34.80 \\
\hline 11 & 9.84 & 20.13 & 3.61 & 44.69 \\
\hline 12 & 8.26 & 14.89 & 1.98 & 43.49 \\
\hline 13 & 14.34 & 27.71 & 2.26 & 38.50 \\
\hline 14 & 8.73 & 17.19 & 1.88 & 44.43 \\
\hline 15 & 7.35 & 11.96 & 1.73 & 41.65 \\
\hline 16 & 4.74 & 6.51 & 2.02 & 43.00 \\
\hline 17 & 13.81 & 23.27 & 2.31 & 44.54 \\
\hline 18 & 8.08 & 16.74 & 3.61 & 46.48 \\
\hline
\end{tabular}

Table 3.2 Mean values and standard deviation of the mechanical properties

\begin{tabular}{|c|c|c|c|c|c|c|c|c|c|}
\hline \multicolumn{2}{|c|}{$\begin{array}{c}\text { Dimension } \\
\text { (inch) }\end{array}$} & \multicolumn{2}{c|}{$\begin{array}{c}\text { UCS } \\
\text { (psi) }\end{array}$} & \multicolumn{2}{c|}{$\begin{array}{c}\text { Young's Modulus } \\
\text { (psi) }\end{array}$} & \multicolumn{2}{c|}{$\begin{array}{c}\text { Cohesion } \\
\text { (psi) }\end{array}$} & \multicolumn{2}{c|}{$\begin{array}{c}\text { Friction } \\
\text { Angle }\left({ }^{\circ}\right)\end{array}$} \\
\hline Length & Diameter & $\mu_{U C S}$ & $S . D_{\cdot U C S}$ & $\mu_{E}$ & $S . D_{\cdot E}$ & $\mu_{c}$ & $S . D_{\cdot c}$ & $\mu_{f}$ & $S . D_{\cdot f}$ \\
\hline 4.35 & 1.99 & $9,450.60$ & $2,931.24$ & $1.74 \times 10^{6}$ & $748,776.95$ & $2,400.37$ & 740.15 & 43 & 3.65 \\
\hline
\end{tabular}

Note:

$\mu$ is the mean value of each property;

S.D. is the standard deviation of each property. 
Table 3.2 summarizes the mean geometric specimen size values and gives standard deviations of four different material property parameters. There is high variation in the five mechanical parameters representing the strength and deformability of the rock specimens.

\subsubsection{Statistical analysis}

Statistical analysis determined the statistical distribution of these parameters. Weibull proposes a statistical approach using the weakest-link theory to explain the inherent variability in strength of structures (Weibull 1939). He finally concluded that the tensile strength of material follows a probability distribution, as shown in Figure 2.1, which was later named the Weibull distribution. Many fields quickly recognized and applied the benefits of this approach across many fields, such as reliability engineering, fatigue, and rock fracture mechanics (Priest \& Hudson, 1976; Einstein, 1983; La Pointe, 1985). The probability density function (PDF) of Weibull distribution is expressed by,

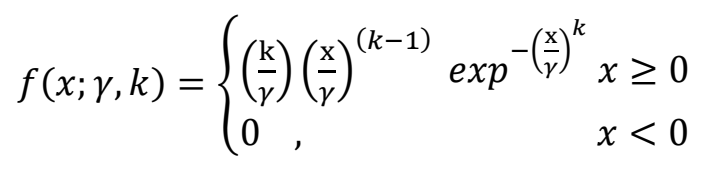

where $k$ is the shape parameter, and $\gamma$ is the scale parameter of the distribution.

From the probability distribution function of the Weibull distribution, zero is one of the boundaries; this boundary is the weakest link in Weibull's theory. However, each site data has its own lower threshold, and zero is not the corresponding threshold value in most cases. Based on Weibull's theory, Yegulalp and Kim analyzed Young's modulus and Uniaxial Compressive Strength datasets and concluded that these mechanical properties followed Type Ш Extreme Value distribution of the smallest value (Yegulalp \& Kim, 1992). The distribution revealed the 
inherent variability in the mechanical properties of the intact rock. The probability distribution of Type Ш Extreme Value distribution of the smallest value can be written as (Gumbel, 1958):

$$
f(x ; \gamma, k, \varepsilon)= \begin{cases}\frac{k}{\gamma-\varepsilon}\left(\frac{x-\varepsilon}{\gamma-\varepsilon}\right)^{k-1} & \exp ^{-\left(\frac{x-\varepsilon}{\gamma-\varepsilon}\right)^{k}}, \\ 0, & x \varepsilon \varepsilon \\ x<\varepsilon\end{cases}
$$

where $\varepsilon$ is the characteristic smallest value of data set $X$. From Equations 3.1 and 3.2, it is notable that the Weibull distribution is a special case of Type III Extreme Value distribution. In other words, Type III Extreme Value distribution of the smallest value is a general form of the Weibull distribution. There are no limitations for the symbol of the smallest value of Type III Extreme Value distribution. For the Weibull distribution, the lowest bound is set to be zero. This is the prerequisite for the definition of Weibull distribution, which is not the case in the reality.

Moreover, the theory of Weibull distribution is only suitable for the tensile strength. The distributions for other strength parameters are still uncertain. In 1994, as shown in Figure 3.1, Yegulalp and Kim performed laboratory tests and concluded that these mechanical properties perfectly fit the Type $\amalg$ Extreme Value distribution of the smallest value not only for the tensile strength, but also for the Young's modulus and the Uniaxial Compressive Strength. Therefore, this study we considered using the Type III Extreme Value distribution as the statistical model in this research. The basic theory of the Extreme Value distribution is as follows: 

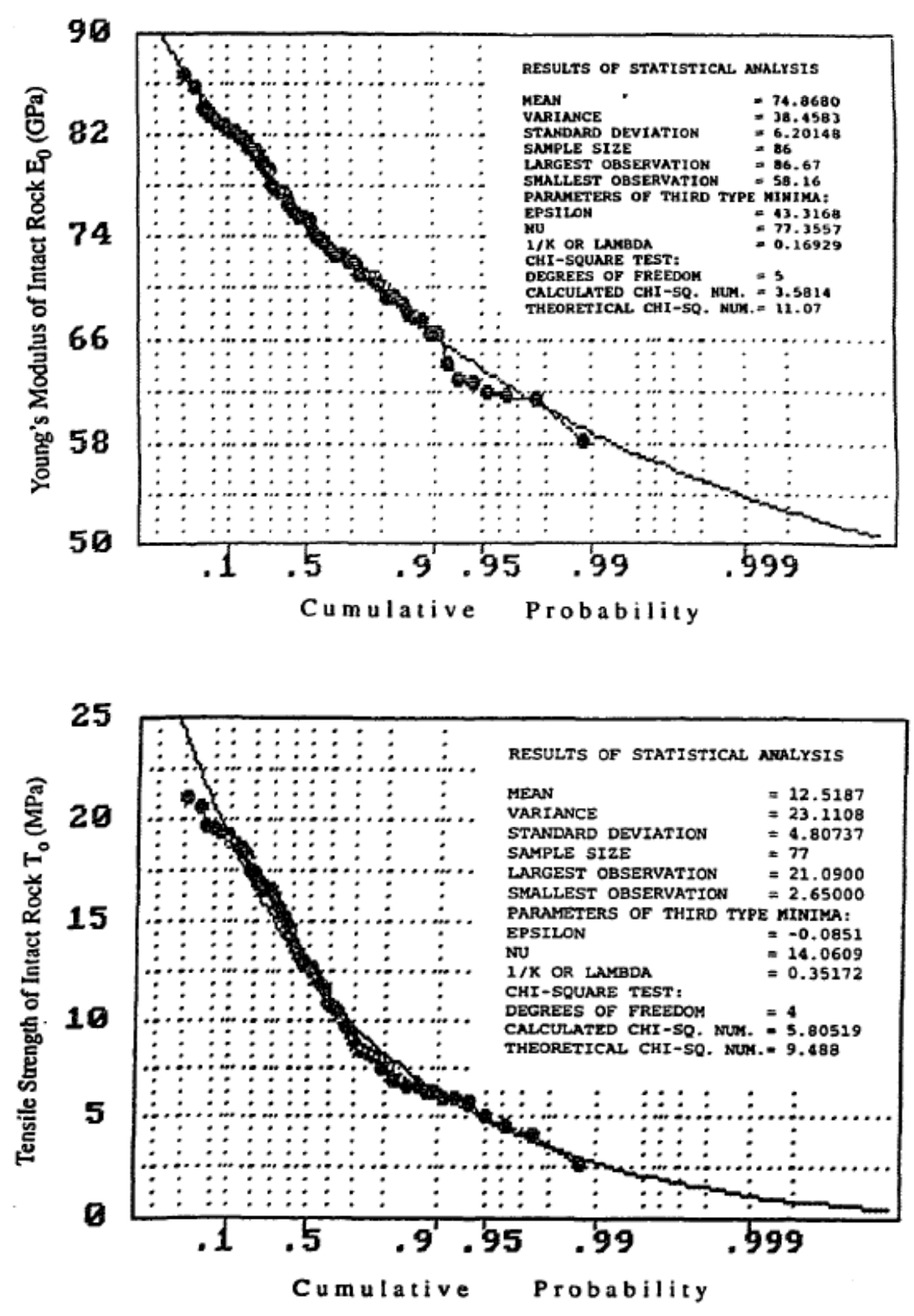


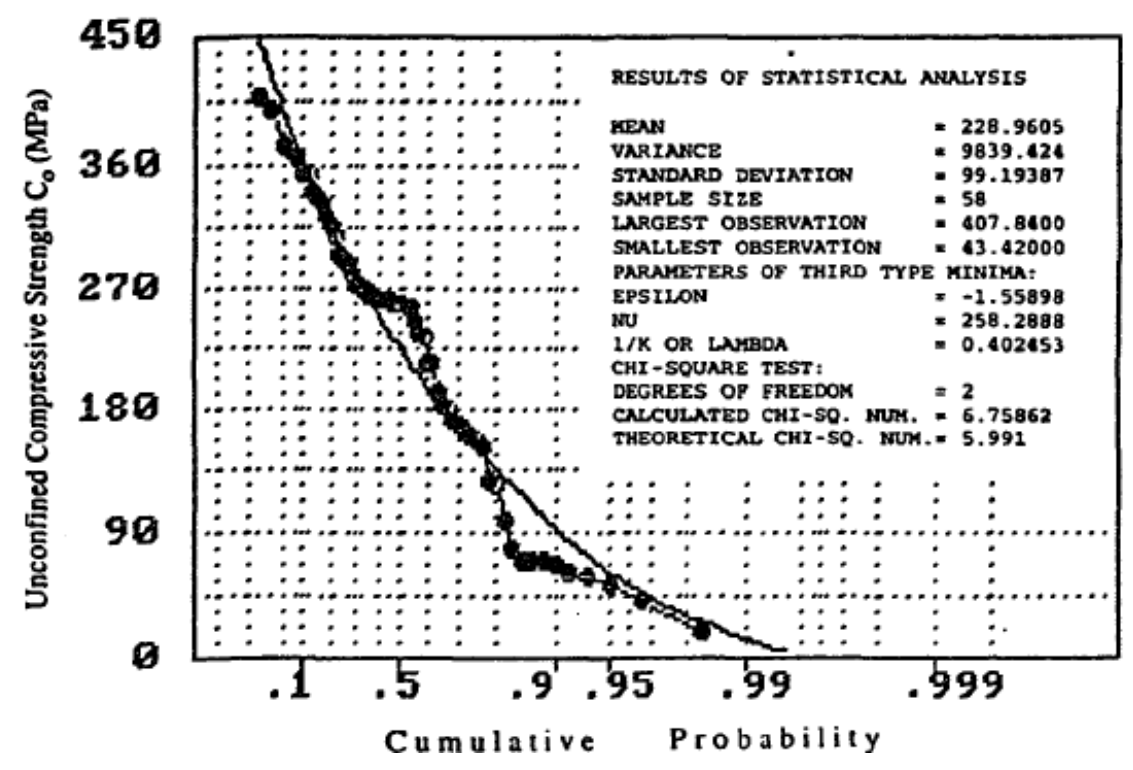

Figure 3.1 Comparison for three different parameters between observed and theoretical extremal distributions (Kim, 1994)

Let $X$ be a random variable set with some known distribution function. If there exist $\mathrm{n}$ samples for the random variable $X$, then the extreme values of the samples can be defined, such as the minimum value $Y_{1}$ :

$$
Y_{1}=\min \left(X_{1}, X_{2}, \ldots, X_{n}\right)
$$

If different sets of samples with the same size $n$ are obtained for $X$, each set will have different minimum values. Using these sets, one can construct distribution functions for the minimum values. The cumulative distribution function (CDF) of the smallest value $Y_{1}$ can be derived, as shown in Equation 3.4:

$$
P\left(Y_{1}>y\right)=P\left(X_{1}>y, \ldots, X_{n}>y\right)=1-F_{Y_{1}}(y)
$$

If all the data sets $X_{1}, X_{2}, \ldots, X_{n}$ are assumed to be identically distributed and statistically independent from one another, Equation 3.4 becomes:

$$
F_{Y_{1}}(y)=1-\left[1-F_{X}(y)\right]^{n}
$$


The basic idea behind these derivations is that, if the smallest value $Y_{l}$ is greater than some quantity $y$, all the samples $X_{1}, X_{2}$, up to $X_{n}$ should be greater than $y$. As the sample size n grows larger and tends to infinity, the distribution of the smallest values will be asymptotically close to a mathematical distribution function in some cases.

Gumbel classifies three types of asymptotic extreme value, labeling them as Type I, II, and III Extreme Value distributions (Gumbel, 2013). Each type is classified into two groups: one group for the largest value, and the other for the smallest value.

The Type III Extreme Value distribution have been used commonly to describe the strength and time of failure of the specimen in mechanical devices and components (Hoek, 1980). This research uses the Type III Extreme Value distribution of the smallest value to evaluate the variation in the mechanical parameters of intact rocks. MATLAB fit the laboratory test results and generated both the random parameters and the Type III Extreme Value distribution of the smallest value (MathWorks, 2017), defined as:

$$
f(x)=\frac{1}{\sigma} \exp \left(\frac{x-\mu}{\sigma}\right) \exp \left(-\exp \left(\frac{x-\mu}{\sigma}\right)\right), x \geq \varepsilon
$$

where $\varepsilon$ is the lower bound of $\mathrm{X}, \sigma$ is the scale parameter, and $\mu$ is the location parameter.

Figure 3.2 shows several curves for Type III Extreme Value probability density function with different scale parameters. Parameter $\sigma$ reflects the material uniformity of the structure for the statistical model. The shape of the statistical distribution changes from flat and wide to tall and narrow with change in $\sigma$. The intensity of the value tends to be uniform, and most of the cells are close to the given location parameter $\mu$. In addition, due to the randomness character the sample values of the material properties generated each time are different, even with the same $\sigma$ and $\mu$ values. 


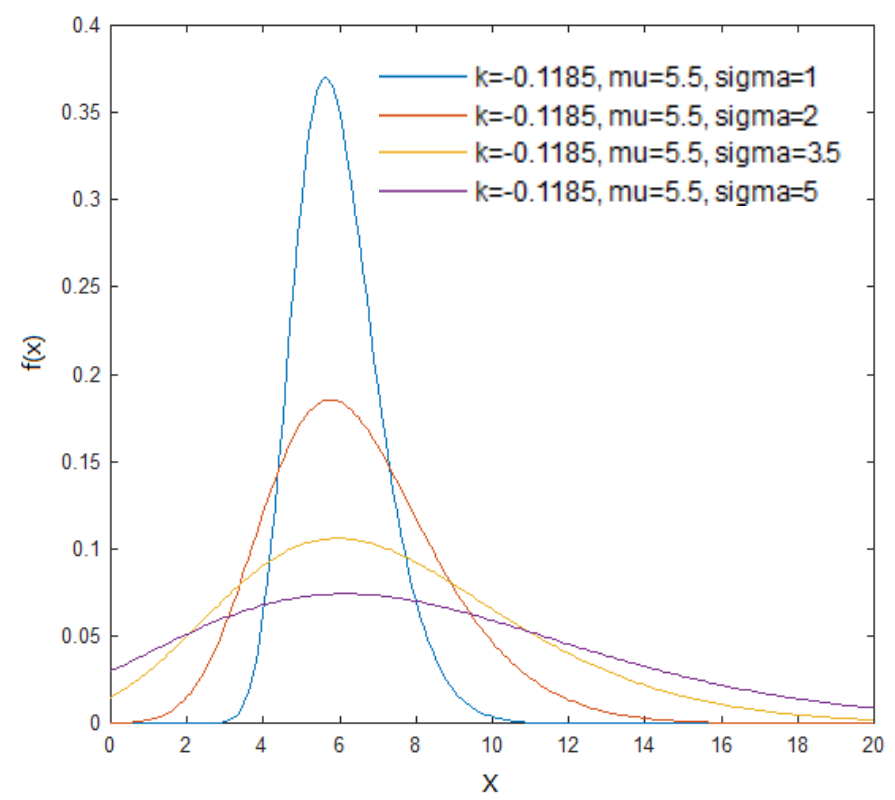

Figure 3.2 Probability density function curves for different indices

In this research, three sets of Uniaxial Compressive Strength (UCS) experimental data obtained from the Pinewood mine were selected. Each data has a different roof depth range, which are:

Group 1: 522.4'-532.35', 18 specimens were tested;

Group 2: 532.35'-542.51', 19 specimens were tested;

Group 3: 542.51'-552.41', 20 specimens were tested.

In order to find the final probability density function (PDF) for Uniaxial Compressive Strength,

$$
f(x)=\frac{k}{\gamma-\varepsilon}\left(\frac{x-\varepsilon}{\gamma-\varepsilon}\right)^{k-1} e^{-\left(\frac{x-\varepsilon}{\gamma-\varepsilon}\right)^{k}}, x \geq \varepsilon
$$

First, one must calculate the three values of $\varepsilon, \gamma$ and $k$.

The mean and variance of $X$ are related to the parameters $\gamma$ and $k$ as follows: 


$$
\mu_{x}=\varepsilon+(\gamma-\varepsilon) \Gamma\left(1+\frac{1}{k}\right)
$$

where $\mu \_x$ is the mean value of $X$, and:

$$
\sigma_{X}^{2}=\operatorname{Var}(X-\varepsilon)=(\gamma-\varepsilon)^{2}\left[\Gamma\left(1+\frac{2}{k}\right)-\Gamma^{2}\left(1+\frac{1}{k}\right)\right]
$$

Equation (4) and (5) can be used to show that

$$
1+\left(\frac{\sigma_{X}}{\mu_{X}-\varepsilon}\right)^{2}=\frac{\Gamma\left(1+\frac{2}{k}\right)}{\Gamma^{2}\left(1+\frac{1}{k}\right)}
$$

Figure 3.3 shows this information graphically.

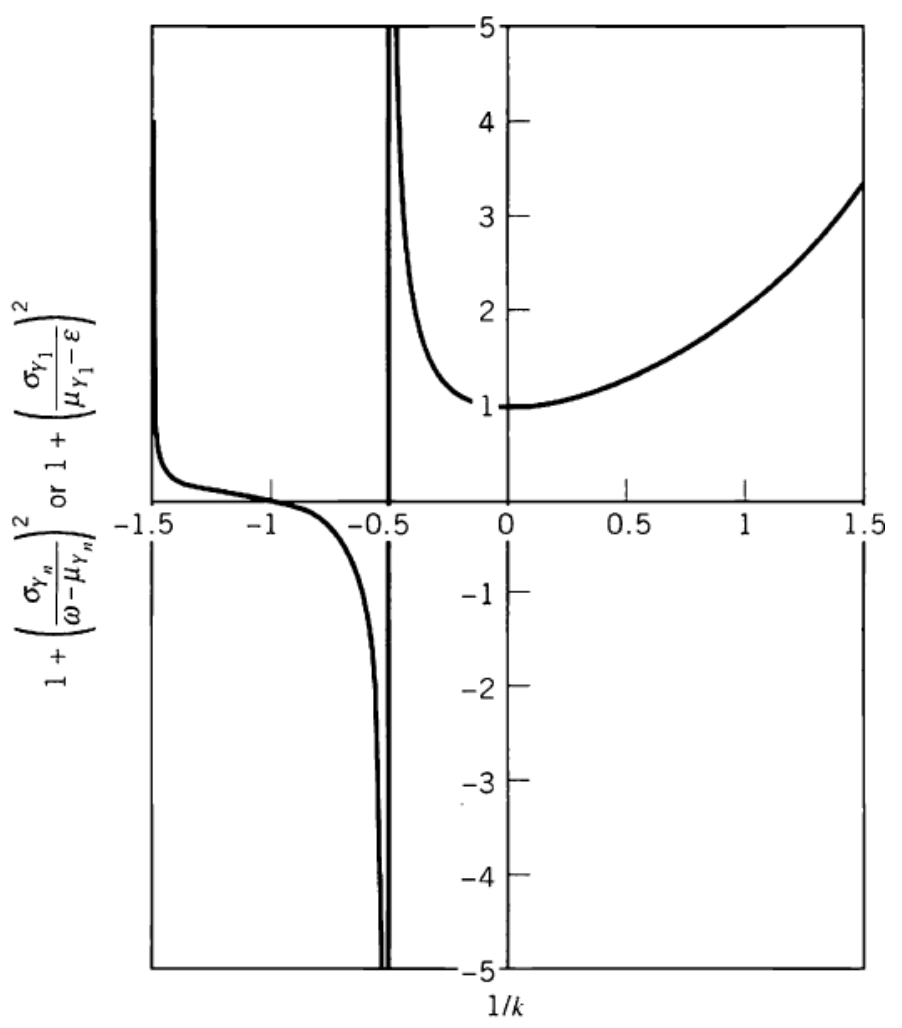

Figure 3.3 Relationship between the Mean and Variance of $Y_{n}$ (or $Y_{l}$ ) and $1 / k$ (Haldar, 2000) (Here, use $Y_{n}$ or $Y_{l}$ instead of $X$, and $Y_{n}$ represents the largest value, $Y_{l}$ represents the smallest value) Here, the first group is used as an example to show the calculation. Table 3.3 is the summary of its UCS. 4,735 is the obvious smallest value, which means the minimum value $\varepsilon=4,735$. 
Table 3.3 Uniaxial Compressive Strength (UCS) for the 1st group. (Unit: psi)

\begin{tabular}{|c|c|c|c|c|c|c|c|c|c|}
\hline No. & $\mathbf{1}$ & $\mathbf{2}$ & $\mathbf{3}$ & $\mathbf{4}$ & $\mathbf{5}$ & $\mathbf{6}$ & $\mathbf{7}$ & $\mathbf{8}$ & $\mathbf{9}$ \\
\hline UCS & 11,141 & 16,120 & 9,391 & 8,024 & 5,961 & 8,868 & 9,616 & 10,975 & 7,160 \\
\hline \hline No. & $\mathbf{1 0}$ & $\mathbf{1 1}$ & $\mathbf{1 2}$ & $\mathbf{1 3}$ & $\mathbf{1 4}$ & $\mathbf{1 5}$ & $\mathbf{1 6}$ & $\mathbf{1 7}$ & $\mathbf{1 8}$ \\
\hline UCS & 7,703 & 9,840 & 8,258 & 14,338 & 8,730 & 7,353 & 4,735 & 13,815 & 8,083 \\
\hline
\end{tabular}

Now, one can calculate the left-hand side of Equation (3.9) as

$$
1+\left(\frac{\sigma_{x}}{\mu_{x}-\varepsilon}\right)^{2}=1+\left(\frac{2,931.381}{9,450.611-4,735}\right)^{2}=1.39
$$

Using Figure 3.3, one can obtain the value of $1 / k=0.58$, or $k=1.724138$. Using Equation (3.7),

$$
\begin{gathered}
\mu_{x}=\varepsilon+(\gamma-\varepsilon) \Gamma\left(1+\frac{1}{k}\right) \\
9,450.611=4,735+(\gamma-4,735) \times 0.891435 \\
\gamma=10,024.91
\end{gathered}
$$

Finally, the PDF function for the first group is obtained:

$$
f(x)=0.000326 \times\left(\frac{x-4,735}{5,289.912}\right)^{0.724138} \times \exp \left(-\left(\frac{x-4,735}{5,289.912}\right)^{1.724138}\right)
$$

Table 3.4 $\varepsilon, \gamma$ and $k$ values for the three groups. (Unit: psi)

\begin{tabular}{|c|c|c|c|}
\hline \multirow{2}{*}{$\begin{array}{c}\text { Number of } \\
\text { Tests }\end{array}$} & \multicolumn{3}{|c|}{ Roof Depth (ft.) } \\
\cline { 2 - 4 } & $\mathbf{5 2 2 . 4}^{\prime} \mathbf{- 5 3 2 . 3 5}$ & $\mathbf{5 3 2 . 3 5}^{\prime} \mathbf{- 5 4 2 . 5 1} \mathbf{\prime}^{\prime}$ & $\mathbf{5 4 2 . 5 1}^{\prime} \mathbf{- 5 5 2 . 4 1} \mathbf{\prime}^{\prime}$ \\
\hline 1 & 11,141 & 10,811 & 4,688 \\
\hline 2 & 16,120 & 11,974 & 11,937 \\
\hline 3 & 9,391 & 9,802 & 12,692 \\
\hline 4 & 8,024 & 10,280 & 12,753 \\
\hline 5 & 5,961 & 6,225 & 11,045 \\
\hline 6 & 8,868 & 9,536 & 12,786 \\
\hline 7 & 9,616 & 7,226 & 5,249 \\
\hline 8 & 10,975 & 9,569 & 9,540 \\
\hline 9 & 7,160 & 9,615 & 15,502 \\
\hline
\end{tabular}




\begin{tabular}{|c|c|c|c|}
\hline 10 & 7,703 & 9,477 & 11,791 \\
\hline 11 & 9,840 & 8,396 & 5,624 \\
\hline 12 & 8,258 & 10,990 & 4,931 \\
\hline 13 & 14,338 & 7,668 & 12,068 \\
\hline 14 & 8,730 & 10,824 & 9,026 \\
\hline 15 & 7,353 & 6,259 & 8,822 \\
\hline 16 & 4,735 & 11,783 & 13,391 \\
\hline 17 & 13,815 & 12,727 & 7,805 \\
\hline 18 & 8,083 & 14,088 & 10,999 \\
\hline 19 & -- & 6,876 & 5,851 \\
\hline 20 & -- & -- & 6,260 \\
\hline Mean & $9,450.61$ & $9,695.58$ & 9,638 \\
\hline $\begin{array}{c}\text { Standard } \\
\text { Derivation }\end{array}$ & $2,931.38$ & $2,190.41$ & $3,318.73$ \\
\hline $\boldsymbol{\gamma}$ & $10,024.91$ & $10,113.87$ & $10,213.06$ \\
\hline $\boldsymbol{k}$ & 1.72 & 1.69 & 1.61 \\
\hline $\boldsymbol{\varepsilon}$ & 4,735 & 6,225 & 4,688 \\
\hline
\end{tabular}

Similarly, for the second and third groups, one can obtain the values of $\varepsilon, \gamma$ and $\mathrm{k}$ as well as the PDF for each group, as shown in Table 3.4 and Table 3.5.

Table 3.5 Probability Density Function (PDF) function for the three groups

\begin{tabular}{|c|c|c|}
\hline $\begin{array}{c}\text { Group } \\
\text { Number }\end{array}$ & Roof Depth (ft.) & Probability Density Function (PDF) \\
\hline 1 & $522.4^{\prime}-532.35^{\prime}$ & $f(x)=0.000326 \times\left(\frac{x-4735}{5,289.91}\right)^{0.724138} \times \exp \left(-\left(\frac{\mathrm{x}-4,735}{5,289.91}\right)^{1.72}\right)$ \\
\hline 2 & $532.35^{\prime}-542.51^{\prime}$ & $f(x)=0.000436 \times\left(\frac{x-6225}{3,888.87}\right)^{0.694915} \times \exp \left(-\left(\frac{\mathrm{x}-6225}{3,888.87}\right)^{1.69}\right)$ \\
\hline 3 & $542.51^{\prime}-552.41^{\prime}$ & $f(x)=0.000292 \times\left(\frac{x-4,735}{5,525.06}\right)^{0.612908} \times \exp \left(-\left(\frac{\mathrm{x}-4688}{5,525.06}\right)^{1.61}\right)$ \\
\hline
\end{tabular}

Figure 3.4 shows the fitting results of the test data with the Type III Extreme Value distribution of the smallest value. The obtained results were good fitting, demonstrating that the 
distribution of the mechanical parameters obeys the Type III Extreme Value distribution of the smallest value.
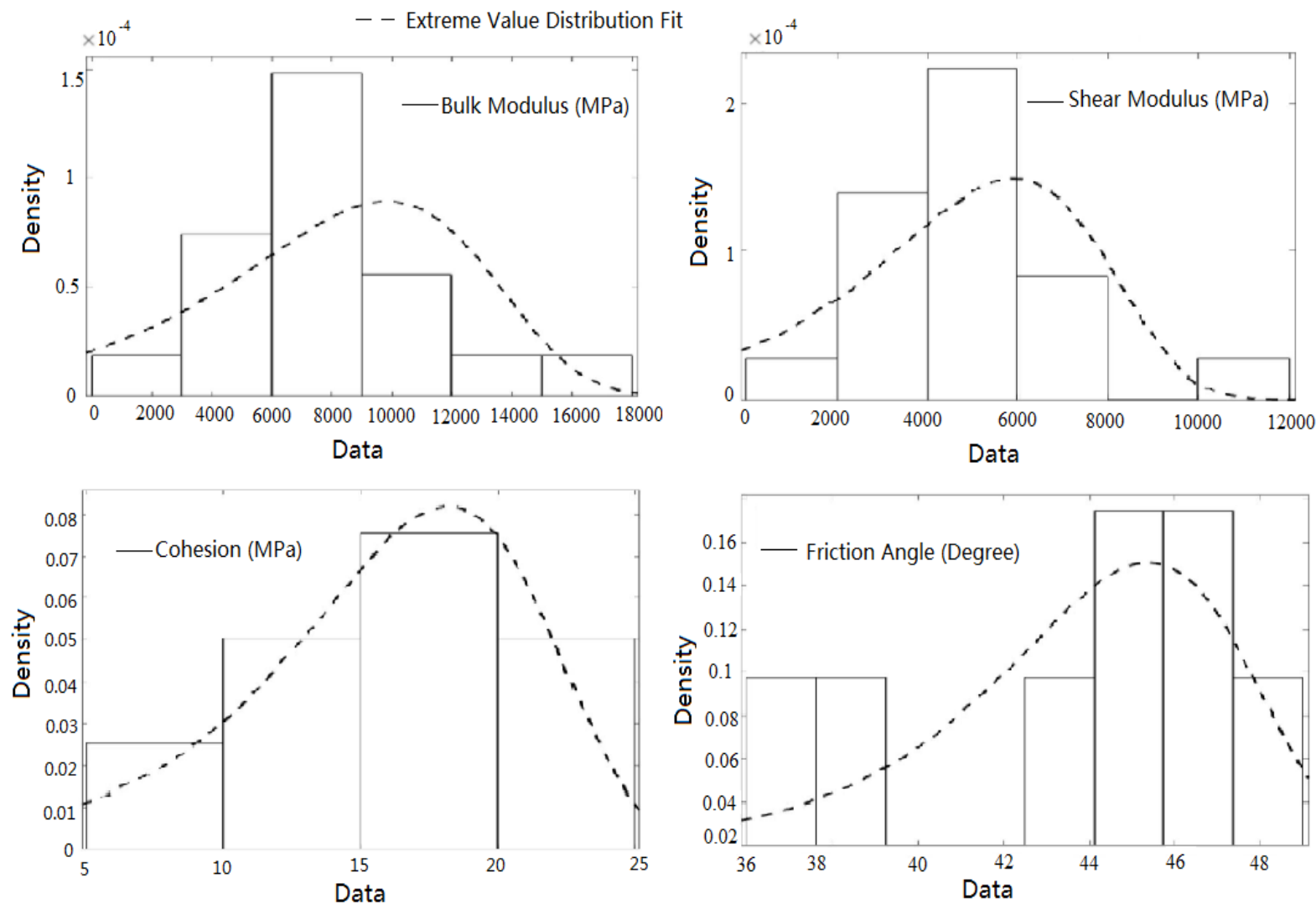

Figure 3.4 Probability density curves of properties of rock core samples

This research uses the eighteenth test results from the first group as its research sample data. Table 3.6 lists the summary of the eighteenth test results for the four different parameters: Bulk modulus, Shear modulus, cohesion, and friction angle. It also includes the determined parameters for each distribution shown in Figure 3.4. 
Table 3.6 Parameters of mechanical properties determined by Extreme Value distribution

\begin{tabular}{|c|c|c|c|c|c|c|c|}
\hline \multicolumn{2}{|c|}{$\begin{array}{l}\text { Bulk Modulus } \\
\left(\times 10^{5} \mathrm{psi}\right)\end{array}$} & \multicolumn{2}{|c|}{$\begin{array}{l}\text { Shear Modulus } \\
\left(\times 10^{5} \mathrm{psi}\right)\end{array}$} & \multicolumn{2}{|c|}{$\begin{array}{l}\text { Cohesion } \\
\left(\times 10^{3} \text { psi) }\right. \\
\end{array}$} & \multicolumn{2}{|c|}{$\begin{array}{c}\text { Friction Angle } \\
\left({ }^{\circ}\right)\end{array}$} \\
\hline \multicolumn{2}{|c|}{16.71} & \multicolumn{2}{|c|}{10.02} & \multicolumn{2}{|c|}{2.57} & \multicolumn{2}{|c|}{40.33} \\
\hline \multicolumn{2}{|c|}{25.45} & \multicolumn{2}{|c|}{15.27} & \multicolumn{2}{|c|}{2.17} & \multicolumn{2}{|c|}{42.78} \\
\hline \multicolumn{2}{|c|}{7.89} & \multicolumn{2}{|c|}{4.73} & \multicolumn{2}{|c|}{2.38} & \multicolumn{2}{|c|}{39.38} \\
\hline \multicolumn{2}{|c|}{7.97} & \multicolumn{2}{|c|}{4.78} & \multicolumn{2}{|c|}{1.90} & \multicolumn{2}{|c|}{43.87} \\
\hline \multicolumn{2}{|c|}{6.39} & \multicolumn{2}{|c|}{3.83} & \multicolumn{2}{|c|}{1.86} & \multicolumn{2}{|c|}{46.45} \\
\hline \multicolumn{2}{|c|}{10.07} & \multicolumn{2}{|c|}{6.04} & \multicolumn{2}{|c|}{4.16} & \multicolumn{2}{|c|}{46.75} \\
\hline \multicolumn{2}{|c|}{13.04} & \multicolumn{2}{|c|}{7.82} & \multicolumn{2}{|c|}{1.43} & \multicolumn{2}{|c|}{47.9} \\
\hline \multicolumn{2}{|c|}{9.78} & \multicolumn{2}{|c|}{5.87} & \multicolumn{2}{|c|}{3.06} & & \\
\hline & & & & & & & \\
\hline & & & & & & & \\
\hline & & & & & & & \\
\hline & & & & & & & \\
\hline & & & & & & & \\
\hline & & & & & & & \\
\hline & & & & & & & \\
\hline & & & & & & & \\
\hline & & & & & & & \\
\hline & & & & & & & \\
\hline$\mu_{K}$ & $\sigma_{K}$ & $\mu_{G}$ & $\sigma_{G}$ & $\mu_{c}$ & $\sigma_{c}$ & $\mu_{f}$ & $\sigma_{f}$ \\
\hline 14.27 & 5.99 & 8.56 & 3.59 & 2.64 & 0.65 & 45.4 & 2.82 \\
\hline
\end{tabular}

Note:

$\mu$ is the location parameter of each property;

$\sigma$ is the scale parameter of each property.

\subsection{Numerical simulation methodology}

Based on the conclusion in the previous study, the Type III Extreme Value distribution of the smallest value is a good fit for the mechanical parameters determined from laboratory compression tests, and for the associated parameters determined within MATLAB. This section proposes a stochastic numerical simulation methodology to simulate the rock specimens with 
randomly generated mechanical parameters. The procedure includes generating random mechanical parameters, assigning the parameters to the elements of the specimen, and numerically simulating an unconfined compression test. In addition, this procedure considers varying element sizes in the simulation.

\subsubsection{Generation of random mechanical parameters}

Figure 3.5 shows the procedure to generate random mechanical parameters in MATLAB. From the test data, one can determine the smallest value $\varepsilon$, the scale parameter $\sigma$, and the location parameter $\mu$ for the distribution. MATLAB can then generate the random parameters, which are a sequence of independent and identically distributed random variables. The number of the property database must be equal to the number of the elements, ensuring the satisfaction of each element by the Type III Extreme Value distribution of the smallest value.

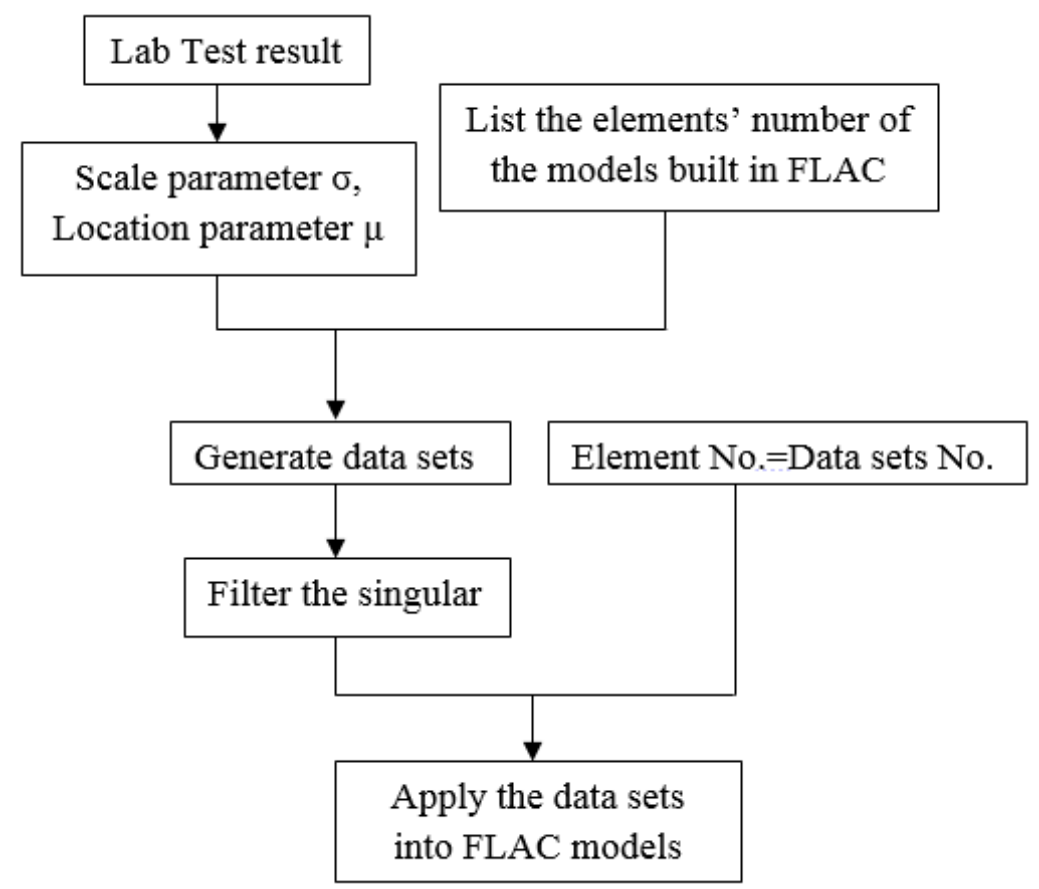

Figure 3.5 Steps to generate database in MATLAB 


\begin{tabular}{|c|c|c|c|c|c|c|}
\hline \multicolumn{7}{|c|}{ Variables - $x 4$} \\
\hline & $x 1 \quad x \quad x 4$ & $x$ & & & & \\
\hline \multicolumn{7}{|c|}{$\boxplus 100 x 100$ double } \\
\hline & 1 & 2 & 3 & 4 & 5 & 6 \\
\hline 7 & $1.1750 e+04$ & $1.1889 \mathrm{e}+04$ & $4.5831 \mathrm{e}+03$ & $5.8970 \mathrm{e}+03$ & $8.4393 e+03$ & $2.1863 \mathrm{e}+$ \\
\hline 8 & $9.3772 \mathrm{e}+03$ & $8.2650 \mathrm{e}+03$ & $9.3458 e+03$ & $5.9085 e+03$ & $1.4836 \mathrm{e}+04$ & $8.3613 e+$ \\
\hline 9 & $1.0575 e+03$ & $7.8492 \mathrm{e}+03$ & $8.6138 \mathrm{e}+03$ & $7.4217 e+03$ & $1.4086 e+04$ & $1.0001 \mathrm{e}+$ \\
\hline 10 & 442.2151 & $7.0620 \mathrm{e}+03$ & $8.9823 e+03$ & $1.2999 \mathrm{e}+04$ & $1.1390 \mathrm{e}+04$ & $8.4306 \mathrm{e}+$ \\
\hline 11 & $1.2914 \mathrm{e}+04$ & $1.0257 e+04$ & $1.2402 \mathrm{e}+04$ & $8.2018 \mathrm{e}+03$ & $9.5292 \mathrm{e}+03$ & $9.3979 \mathrm{e}+$ \\
\hline 12 & -127.4939 & $1.3844 \mathrm{e}+04$ & $1.1549 \mathrm{e}+04$ & $9.6438 \mathrm{e}+03$ & $8.2609 \mathrm{e}+03$ & $8.3416 \mathrm{e}+$ \\
\hline 13 & $1.0832 e+03$ & $1.2200 \mathrm{e}+04$ & $1.0077 e+04$ & -398.3493 & $1.0631 e+04$ & $9.4058 \mathrm{e}+$ \\
\hline 14 & $9.9474 e+03$ & $3.3842 e+03$ & $1.2186 \mathrm{e}+04$ & $8.3227 e+03$ & $5.8615 e+03$ & $7.4404 \mathrm{e}+$ \\
\hline 15 & $6.2269 \mathrm{e}+03$ & $1.2971 \mathrm{e}+04$ & $5.3578 \mathrm{e}+03$ & $6.2260 e+03$ & $7.4763 e+03$ & $9.6075 \mathrm{e}+$ \\
\hline$\cdots$ & ¿aman n. & rare m & $\underset{\text { III }}{. a r a n} n$ & מamen n & mans & man \\
\hline
\end{tabular}

Figure 3.6 Bulk Modulus database generation and filtration in MATLAB

It is important to note that the procedure includes a filter process in order to eliminate the invalid parameters. Figure 3.6 shows a screenshot of the database for Bulk modulus generated in MATLAB. Type III Extreme Value distribution limits the distribution for the lowest value, which can take positive or negative values. Due to this property, the database generated here also includes negative values, which is not realistic. Therefore, it is crucial to filter the invalid values (the values marked with red rectangles) before inputting the data into the numerical models.

\subsubsection{Numerical simulation method}

FLAC conducts numerical simulations with a stochastic method. FLAC uses an explicit finite difference method to capture the complex behaviors of models that consist of several stages, show large displacements and strains, exhibit non-linear material behavior, or are unstable. This includes cases of yield or failure over large areas or total collapse. This process uses MohrCoulomb criterion as the failure criterion, which means that the elements demonstrate perfect plastic behaviors when failure occurs.

Figure 3.7 shows the geometry of the model in 2D and 3D. The figure shows the model in an isometric projection, indicating the $3 \mathrm{D}$ shape. The $2 \mathrm{D}$ model is 4 inches in length and 2 inches in 
width. The 3D model is 4 inches in length and 2 inches in diameter. The dimensions of the model remain constant in the entire modeling effort.

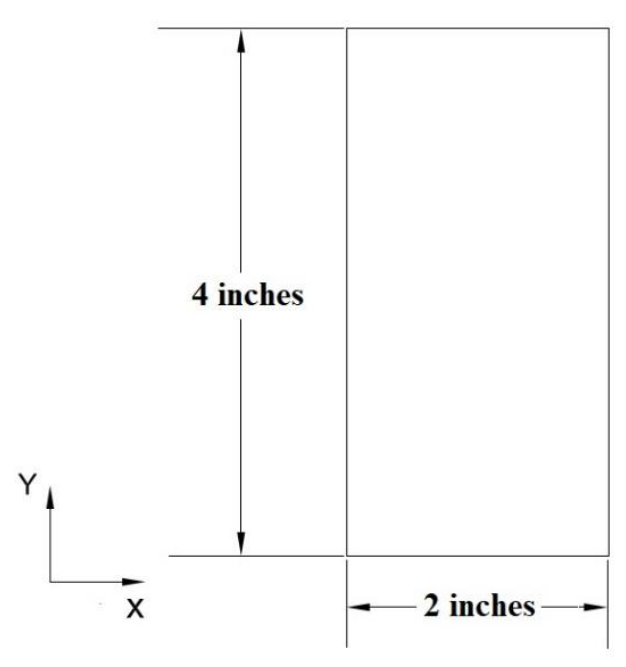

2D model geometry

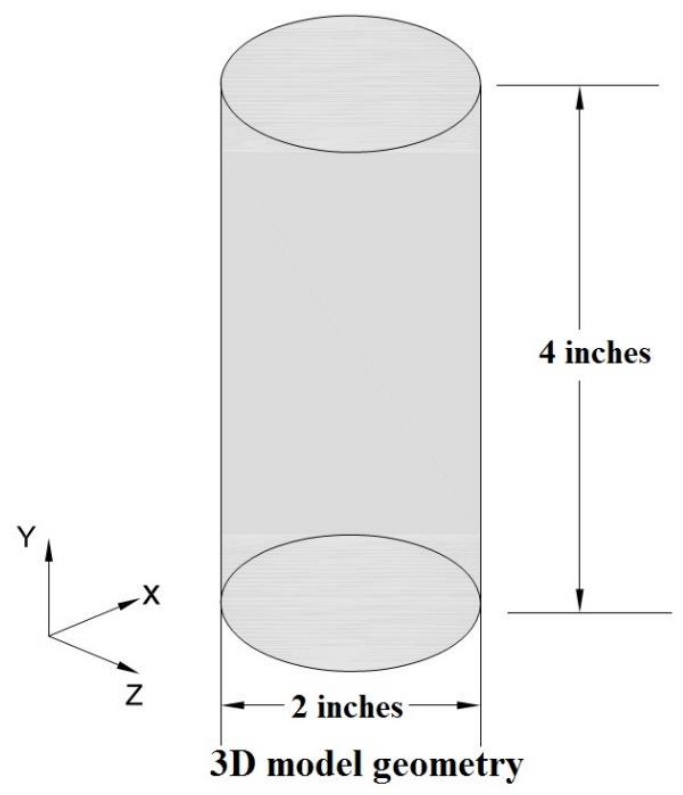

Figure 3.7 Dimensional sketch for the numerical models

Table 3.7 summarizes the peak strengths with three different methods: Type III Extreme Value distribution, Weibull distribution, and the traditionally deterministic method. Figure 3.8 shows the corresponding plot of the peak strengths for those three methods. From the table and figure, the results show the peak value increases as the element number increases; the larger the element number, the more the peak strengths are close to the laboratory results. Here, the laboratory test result of peak strength is $72,046.18 \mathrm{psi}$.

Table 3.7 Peak strengths of rock specimens for different distributed material properties (Unit: psi)

\begin{tabular}{|c|c|c|c|}
\hline $\begin{array}{c}\text { Element } \\
\text { Number }\end{array}$ & $\begin{array}{c}\text { Type III } \\
\text { Extreme Value } \\
\text { distribution }\end{array}$ & $\begin{array}{c}\text { Weibull } \\
\text { distribution }\end{array}$ & $\begin{array}{c}\text { Average } \\
\text { distribution }\end{array}$ \\
\hline 27,000 & $32,983.28$ & $30,183.34$ & $36,902.82$ \\
\hline 125,000 & $40,376.74$ & $37,025.92$ & $46,751.49$ \\
\hline 216,000 & $43,565.76$ & $39,770.03$ & $51,124.53$ \\
\hline 729,000 & $55,921.75$ & $52,689.68$ & $68,593.97$ \\
\hline
\end{tabular}


Moreover, if the model is under the same element number condition, peak strength obtained by Type III Extreme Value distribution is relatively closer to the average distributed model than the Weibull distributed model. The Weibull distribution method used to make the stochastic analysis is still a little too conservative; as such, Type III Extreme Value distribution is an optimal choice for this case.

The simulations use a series of 2D and 3D model configurations, as follows:

a) The first group includes four models with same element numbers: the simulation assigns one of the four models with deterministic mechanical parameters and assigns the other three models with random mechanical parameters. There are 800 elements for the $2 \mathrm{D}$ model and 64,000 elements for the 3D model.

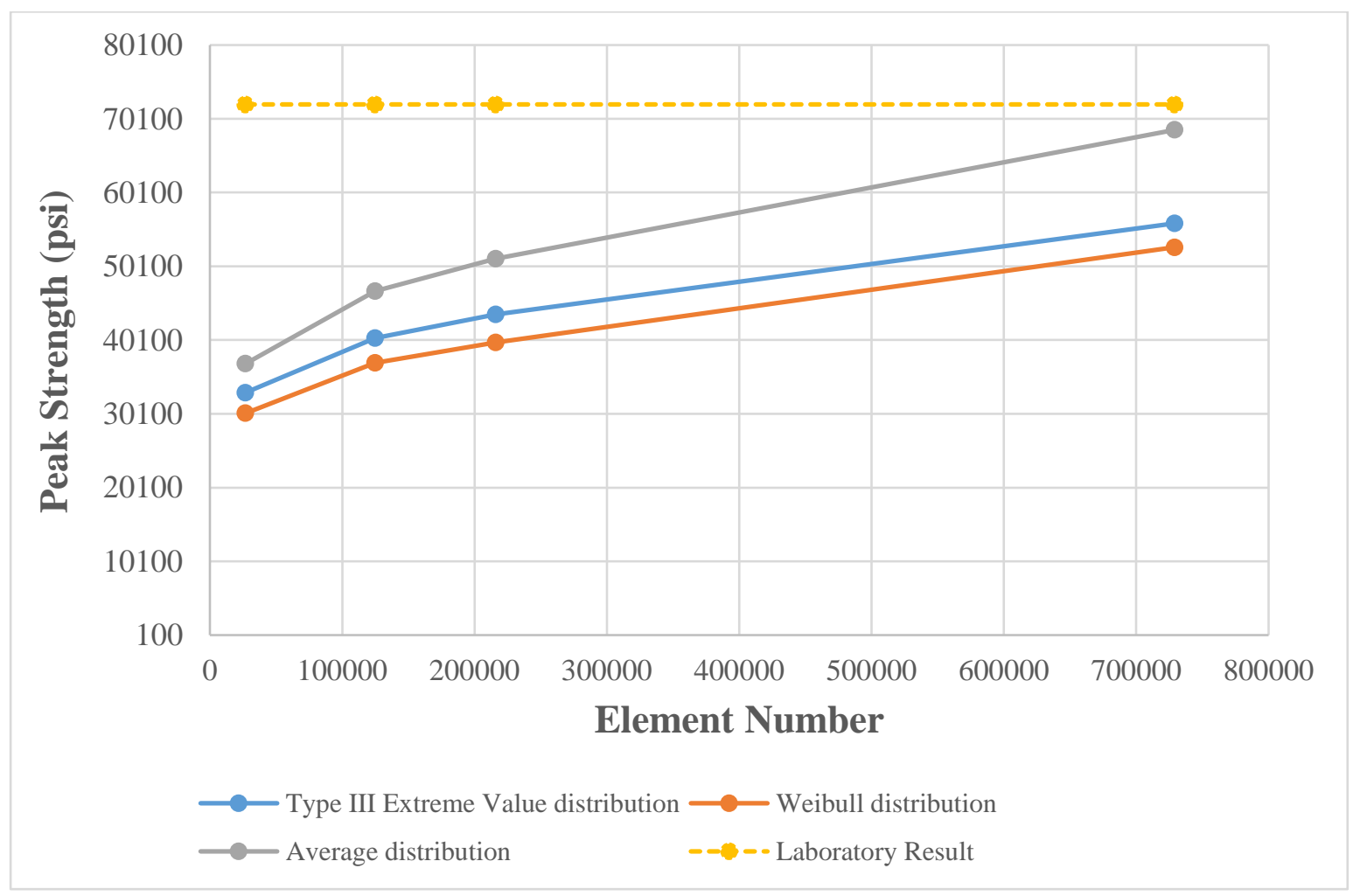

Figure 3.8 Peak strengths of rock specimens for different distributed material properties (Unit: psi) 
b) The second group includes three models with different element numbers: 600, 800, and 1,000 for the 2D models, and $64,000,144,000$, and 400,000 for the 3D models. The random mechanical parameters are the same for all the models in this group.

The program randomly generates mechanical parameters based on the statistical distribution and determined distribution parameters for a large number of elements. Therefore, a written FISH function assigns the random parameters to the elements in the numerical models. For each parameter, the program stores the random data generated and filtered in MATLAB into a .txt file; later, the Fish function reads this data. In other words, the program stores the random data into an array defined in the Fish function. The Fish function then assigns this data to the elements based on the element number. Figure 3.9 shows the cohesion distribution in FLAC8.0 program and Bulk modulus distribution in FLAC3D as examples. The program randomly generates the property with the Type III Extreme Value distribution of the smallest value.

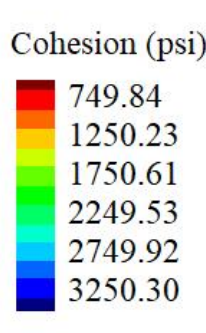

(a) 2D model

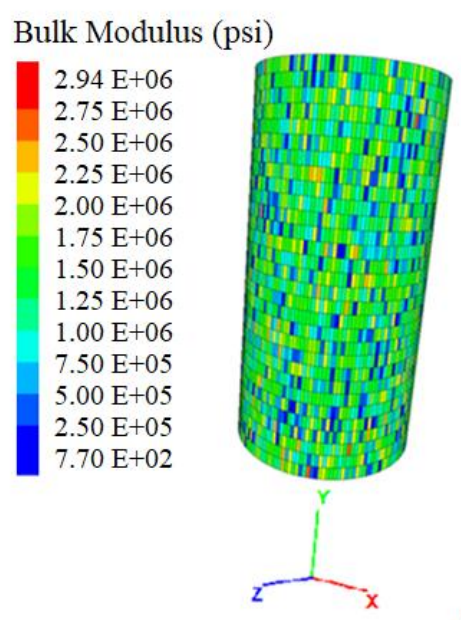

(b) $3 \mathrm{D}$ model

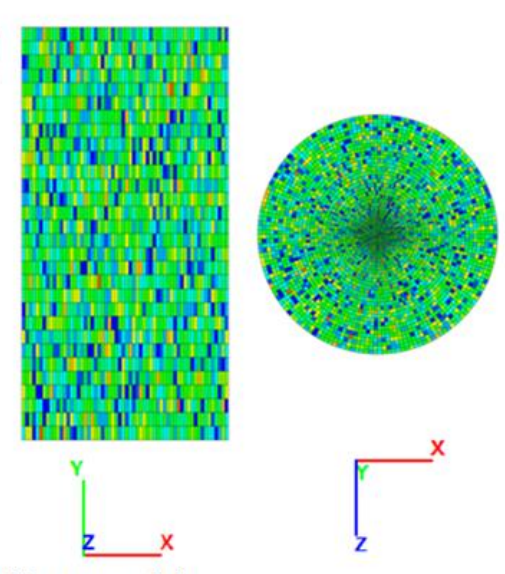

\begin{abstract}
(cohesion) and $3 \mathrm{D}$
\end{abstract}
Figure 3.9 Randomly distributed property schematic diagrams in 2D (cohesion) and 3D (Bulk modulus) models.

Applying velocity to the top of the specimens simulates the unconfined compression test after generating the model and assigning the constitutive model and random properties. The velocity applied to the top of the model is $9.8 \times 10^{-7} \mathrm{inch} / \mathrm{sec}$. The $2 \mathrm{D}$ models have the top of the 
model boundary fixed in the $X$ direction and the bottom boundary fixed in both the $X$ and $Y$ directions. The 3D model has the model fixed in the $X$ and $Y$ directions on the top and in the $X$ and $Z$ directions on the bottom boundary. The model is free to move in the $Y$ direction. When the model is running, the program monitors the force applied to the specimen and the specimen displacement.

\subsection{Results and discussions}

\subsubsection{Influence of heterogeneity on rock behavior}

Rock heterogeneity affects the deformability of the rock specimen. Figure 3.10 shows the stress-strain curves of the 2D and 3D models with deterministic and stochastic properties. From Figure 3.10(a), relationships at pre-failure situation were similar, but slight differences still exist.

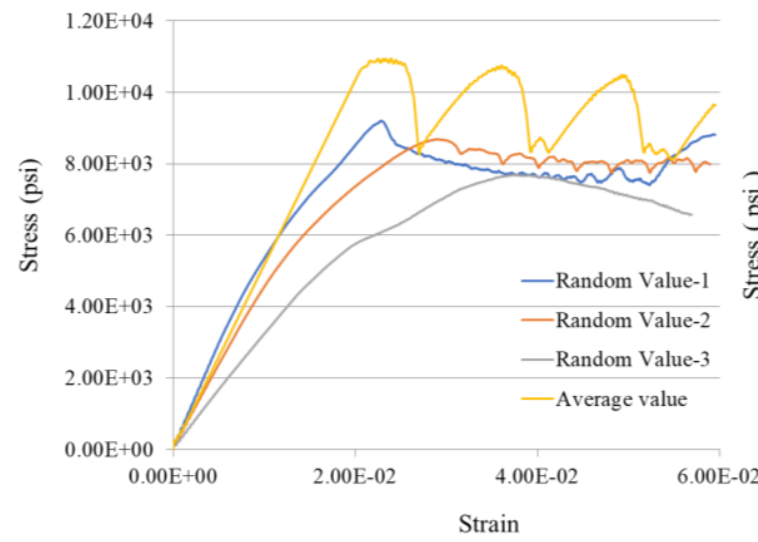

(a) 2D model

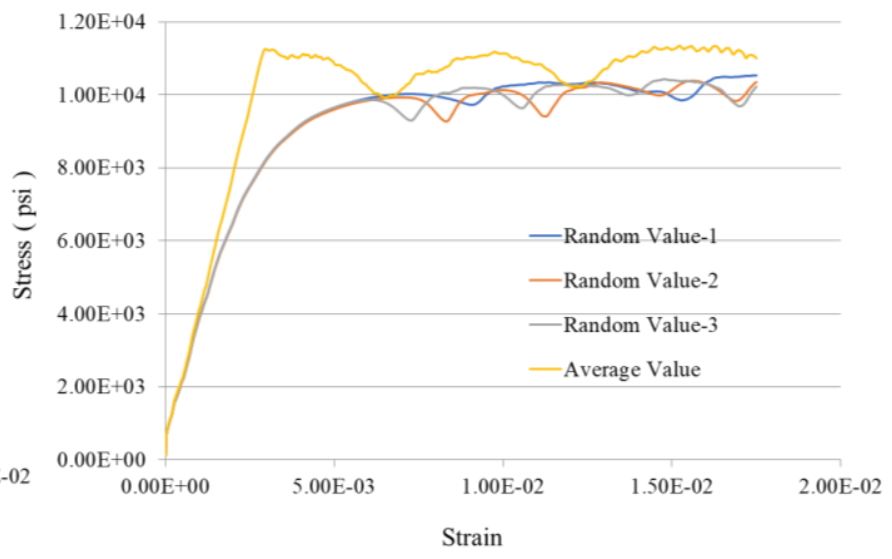

(b) 3D model

Figure 3.10 Stress-strain curves for 2D and 3D models in same element number conditions (Note: Random Value-1, Random Value-2, and Random Value-3 represent three random models assigned with three different random mechanical parameters. The other conditions, like the element numbers, are stochastic parameters for each mechanical property and are kept the same.)

The slope of the curves before the yield point should be same, as the Young's modulus is constant theoretically. Because of this error between the numerical model and theoretical model, it is necessary to build the 3D models. Figure 3.10(b) shows the force-displacement curves in 
FLAC8.0. The slopes for force and displacement curves are obtained that they are the same in the elastic part. The reason for the difference in the $2 \mathrm{D}$ models is mainly due to ignoring the dimension in the $Z$ direction, which played a significant role in the rock's deformation behavior.

With an increasing load applied onto the specimens, the difference between stress-strain curves shown in Figure 3.10 becomes larger and larger, demonstrating that there is a difference in the deformability of the specimen. Due to the heterogeneity in the Bulk and Shear modulus, the displacement distribution within the specimen is inhomogeneous. Some areas within the specimen could have lower modulus and are easier to deform, while others are more difficult to deform. When applying a load onto the specimen, this heterogeneity can induce inhomogeneous deformation within the specimen, which changes its overall deformability.

In addition, the heterogeneity affects the failure process of the specimens. Figure 3.10 shows that the stress-strain curves for the specimens with deterministic properties are almost linear in the pre-failure region. When the load increases to a specific value, all the elements become plastic at the same time. However, the failure of the heterogeneous specimens is different. As seen in Figure 3.10(a), the stress-strain curve is linear at the beginning and gradually becomes nonlinear with increasing load. This phenomenon is the result of the heterogeneity of strength, which induces the progressive failure process.

Figure 3.11 shows the plot of the element state for the specimens with a deterministic property and random values when the applied load is 5000 psi.

Similarly, failed state in 3D shows the random failure distribution in the simulated specimen (Figure 3.12). At the same load level, combining 
Figure 3.11(b) and Figure 3.12, there is no failed element in the specimens with a deterministic property, while some elements have already failed in the specimens with a stochastic property. With increasing load, more and more elements in the specimens with a stochastic property fail, resulting in progressive failure of the specimen and increasing deformability.

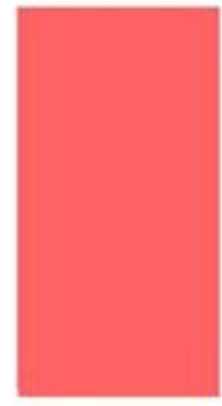

(a) Specimen with deterministic property

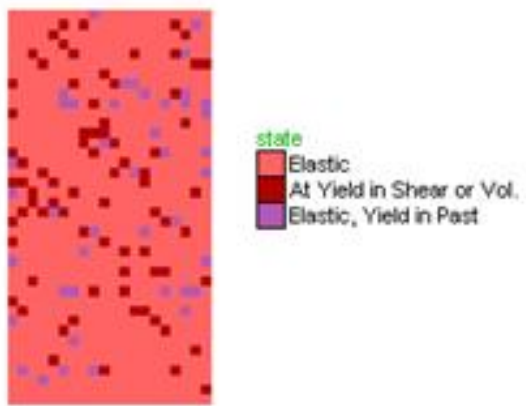

(b) Specimen with random property

Figure 3.11 Plot of element state with different properties

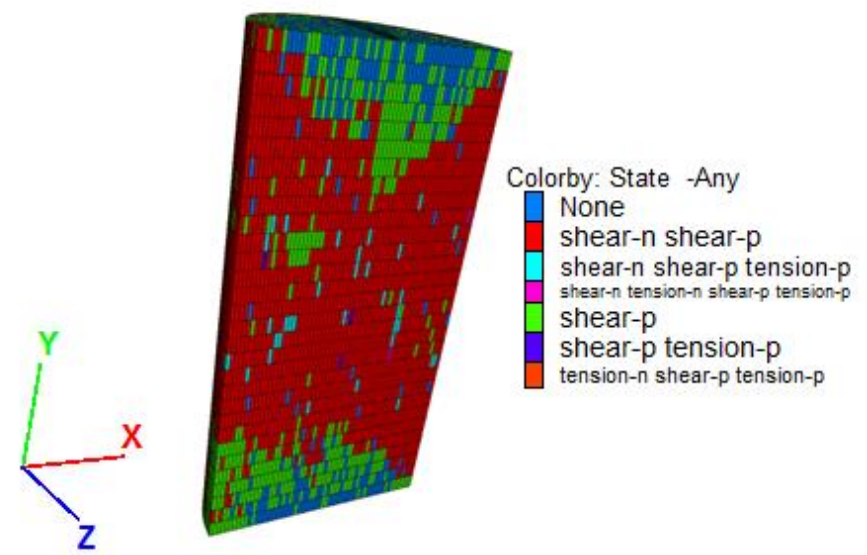

Figure 3.12 Plot of element state with stochastic properties in a three-dimensional rock specimen

Table 3.8 Peak strength for 2D and 3D models with same element number conditions.

(Unit: psi)

\begin{tabular}{|l|c|c|}
\hline & 2D Model & 3D Model \\
\hline Deterministic & $10,950.37$ & $11,254.95$ \\
\hline
\end{tabular}




\begin{tabular}{|c|c|c|}
\hline Random-1 & $9,202.66$ & $8,249.76$ \\
\hline Random-2 & $8,689.23$ & $8,219.30$ \\
\hline Random-3 & $7,689.91$ & $8,168.54$ \\
\hline
\end{tabular}

Finally, the property affects the peak strength of the specimens. Table 3.8 summarizes the peak strength of the $2 \mathrm{D}$ and $3 \mathrm{D}$ specimens. For the $2 \mathrm{D}$ models, a significant decrease in peak strength occurs when using a stochastic property rather than a deterministic property. In addition, the peak strengths are different when assigning different random properties. For the 3D models, a decrease in peak strength also occurred, but the difference between different random properties was negligible. Therefore, the peak strength for the specimens with a stochastic property is lower than that of the specimens with a deterministic property, whether it is a $2 \mathrm{D}$ or $3 \mathrm{D}$ condition.

\subsubsection{Sensitivity analysis of element size on the simulation}

The sensitivity of the element size could possibly affect the simulation results in this research. The simulation can simulate different numbers of elements for one rock specimen, which introduces different element sizes. This process randomly generates the strength and deformability of each element, meaning that they are different from one another. The failure of one element or difference in the deformability of one element, when compared to the surrounding elements, can change the stress distribution for the local area within the specimen. If the sizes of the elements are different, the affected area can be different as well; as a result, the overall response of one specimen to the increasing load can be different. Thus, it is necessary to conduct a sensitivity analysis to analyze the influence of element size on the simulation result. 


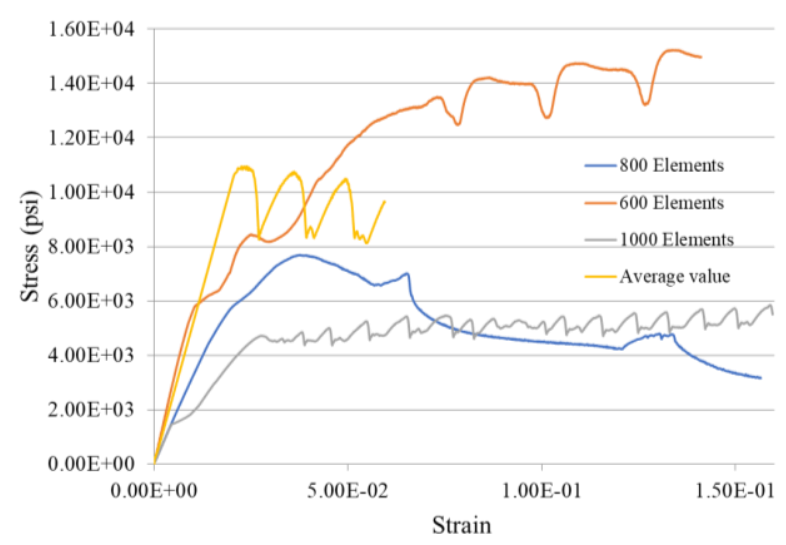

(a) 2D model

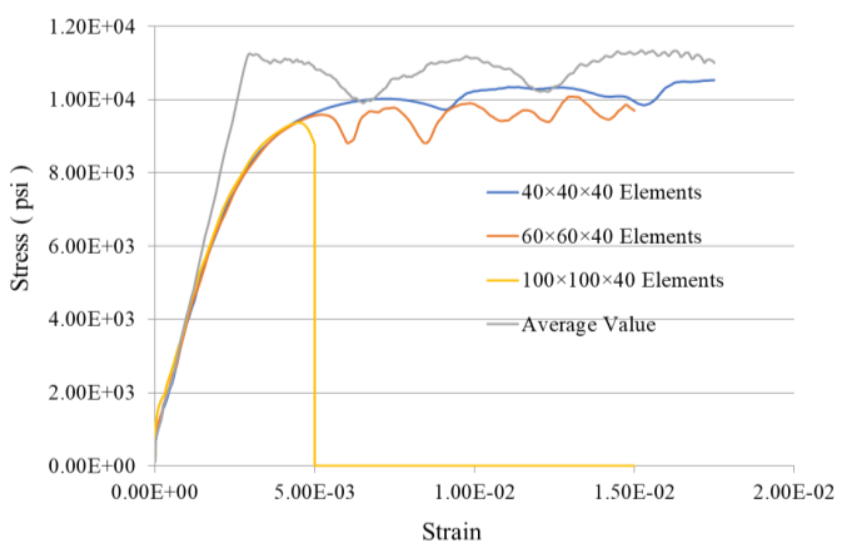

(b) 3D model

Figure 3.13 Stress-strain curves for 2D and 3D models in different element number conditions (Note: ** Elements in this figure represent that six different random models were created with 600, 800, and 1,000 element numbers in FLAC8.0 and 64,000, 144,000, and 400,000 in FLAC3D respectively. The stochastic parameters for each mechanical property are kept the same.)

Table 3.9 Peak strength for $2 \mathrm{D}$ and 3D models with different element number conditions (Unit: psi)

\begin{tabular}{|c|c|c|c|}
\hline \multirow{2}{*}{ 2D model } & $\mathbf{6 0 0}$ Elements & $\mathbf{8 0 0}$ Elements & 1000 Elements \\
\cline { 2 - 4 } & $8,447.01$ & $7,689.91$ & $5,462.13$ \\
\hline \hline \multirow{2}{*}{ 3D Model } & $\mathbf{6 4 , 0 0 0}$ Elements & $\begin{array}{c}\mathbf{1 4 4 , 0 0 0} \\
\text { Elements }\end{array}$ & $\mathbf{4 0 0 , 0 0 0}$ Elements \\
\cline { 2 - 4 } & $8,262.81$ & $8,207.70$ & $8,183.04$ \\
\hline
\end{tabular}

Section 3.3.2 discussed the different numbers of elements used in the numerical simulation of a rock specimen 4 inches in length and 2 inches in diameter. Figure 3.13 shows the stress-strain curves for the $2 \mathrm{D}$ and $3 \mathrm{D}$ models with different element sizes, and Table 3.9 summarizes the peak strengths. For the 2D models, the influence of element size on rock behavior is significant. With an increasing element number or decreasing element size, the slope of the overall stressstrain curve decreases; this indicates an increase in the deformability. In addition, the peak strength decreases with an increasing element number. This demonstrates that the element size significantly influences the simulation results in the $2 \mathrm{D}$ model; as such, the model should be 
calibrated carefully before simulation. However, the element size has a negligible influence on the simulation results in the 3D model, which is notable in the stress-strain curves and peak strengths.

\subsection{Conclusions}

The focus of this chapter was to demonstrate that heterogeneity and discontinuity significantly affect rock strength. Therefore, it is important to use a stochastic analysis in order to determine rock strength. The Type III Extreme Value distribution of the smallest value is the primary methodology. The conclusions of this research are as follows:

Use of the deterministic method results in a conservative estimation of rock strength. Use of the deterministic method overestimates the rock strength by $50 \%$. The peak strength decreases as the element number of the random model increases.

For the deterministic model, the failure always behaves like a double pyramid type of failure. The yield zones are relatively concentrated. For the stochastic models in this research, researchers can monitor both the macro-cracks and the micro-cracks. Each micro-crack will cause stress redistribution and propagate, which will in turn cause a severe failure. As such, the failure pattern for rock specimen with stochastic analysis is less predictable.

The residual strength for the intact rock varies extensively with the grid number. The optimum grid number is unknown, and researchers will investigate this number in the future for more realistic mechanical behaviors of intact rock.

The stochastic models produce more realistic rock behavior. The yield state shows that random failure forms in the stochastic models, which is different from the traditional 
deterministic method. In addition, while strengthening random density, the randomness of the failure element appears more obviously, revealing the nature variability of rocks. 


\section{CHAPTER 4 \\ EFFECT OF SPATIAL VARIANCE FACTOR ON RANDOM MODEL}

\subsection{Introduction}

In rock engineering, accurate strength prediction of rockmass is imperative for a successful design, especially for an underground structure. Complex depositional activity in the rock formation process causes the rock to be heterogeneous. Heterogeneity affects the physicalmechanical behavior of rock. The inherent variability of rock is one of the major sources of uncertainties, commonly observed in the strength variation of intact rocks. The deterministic approach is widely used in solving rock mechanics problems. The single mean values of limited site data have been used to estimate the intact rock parameters such as the strength and deformation modulus (Amusin, 1979; Bieniawski, 1978; Hoek, 1980). However, the estimations are always questionable, as the deterministic methods ignore the inherent variation in the intact rock.

In structures of rocks, the weak formation, the area, which has lower material properties, interacts on a different spatial correlation length. However, few research efforts have focused on the influence of the spatial variance on the stress field investigation and failure procedure of the materials. For example, the strength of pillars in underground mines is critical for mining operations in underground coal and hard rock mines. However, only a unit value is usually considered when performing stability calculations. A significant research effort in past studies examined pillar stability and design using theoretical formulation (Bieniawski, 1968), numerical simulation (Recio-Gordo, 2012), and empirical method (Soltani, 2015). The problem is that these studies always used deterministic strength values to represent the coal strength of the whole 
pillars, ignoring the inherent variability that exists in rockmass properties. In order to analyze rockmass behavior, one must probabilistically assess the inherent variability of rockmass mechanical properties, such as the strength, deformation properties, and in-situ stresses, for the whole area.

In the past few years, random field theory to model the inherent rock variability have been increasingly used in order to reduce uncertainty in rock characteristics. Several researchers such as Weibull used weakest-link theory to explain the inherent variability of a material (Weibull, 1939). Salamon, Peng, and Scovazzo used finite element method, which included variable material properties, on applications such as the pillar design in underground mining (Salamon, 1967; Scovazzo, 1992; Peng, 1992). Priest, Hudson, etc. discovered that the distributions of joints in rockmass follow the exponential distributions (Einstein, 1983; La Pointe, 1985; Priest, 1976). Griffiths used finite element method in Monte Carlo framework and combined the Uniaxial Compressive Strength and spatial correlation to analyze the mine pillar stability (Griffiths, 2002). Yegulalp and Kim showed that the mechanical properties of intact rock match the Type III Extreme Value distribution of the smallest value (Yegulalp \& Kim, 1994). The grain sized rock material properties are typically assigned with random models. However, due to the geology and history of deposition in rock laminations, the formation of rockmass necessitates the random distributions of each physical indicator to be spatially correlated, where the physicalmechanical properties of rockmass are neither deterministic nor completely random. Few studies have considered the spatial correlation factor combined with random rock material properties.

In this chapter, a probabilistic approach is proposed by using Extreme Value distribution and spatial correlation factor, which will assess the variability of the rockmass mechanism. This method treats rockmass properties as spatially correlated random variables. This chapter studies 
three different laboratory scaled models to demonstrate the necessity of considering the spatial correlation factor.

\subsection{Methodology}

Weibull is the first person to propose a statistical approach, using the weakest-link theory to explain the inherent variability in strength of structures; this has been widely applied in the random field analysis for rockmass (Einstein, 1983; Fang, 2002; Kim \& Gao, 1995; Potvin, 2012). Based on Weibull's research, Yegulalp and Kim concluded that Young's modulus, shear modulus, and Uniaxial Compressive Strength of rock followed the Type Ш Extreme Value distribution of the smallest value better than Weibull's distribution (Yegulalp \& Kim, 1992). Type III Extreme Value distribution is the general form of Weibull distribution (Gao, 2020).

Previous research has analyzed the inherent variability of the material property in rocks. However, these researchers only considered the mean value and standard deviation in rock strength, ignoring the effect of spatial correlation. Due to the geology and history of deposition in rock laminations, spatial variability is a significant factor in the mechanical investigation of rocks. The properties of the adjacent areas correlate with each other.

The traditional Extreme Value distribution models only consider the randomness attributes of rockmass. However, due to the geology and history of deposition in rock laminations, the physico-mechanical properties tend to be independent at far distances and correlated at relatively small distances (Ledvina, 1991). The mechanical behavior of a rock at a close distance always shows some spatial continuities and correlations; as such, the spatial variability is a significant factor in the mechanical investigation of rocks. The front view shown in Figure 4.1 is an example 
of this correlation. The local spatial continuities exist inherently within the rock, causing the physical-material properties in adjacent areas to be similar and spatially correlated.
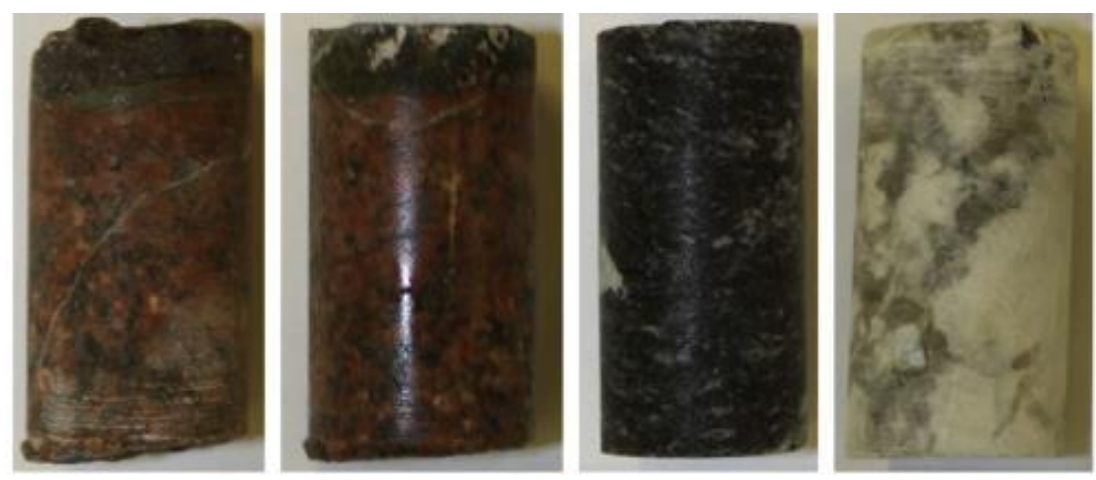

Figure 4.1 Existence of spatial variance in different types of rock specimens (Recio-Gordo, 2012)

Spatial correlation measures the correlation of a variable through space. It is an index value revealing the points, which are significantly correlated within some specific distance, which names the correlation. Figure 4.2 represents one strength variation at different depths. $\bar{\mu}$ is the mean value. $\mu\left(z_{j}\right)$ is the actual strength value at the specific depth. $\sigma_{\mu}$ is the standard deviation for all strength data. $\delta \mu$ is the correlation length, which is the distance of both the two points above or below the mean value $\bar{\mu}$.

For example, as shown in Figure 4.2, take two rock specimens selected at far apart locations. One is from circle $\mathrm{A}$, and one is from circle $\mathrm{B}$, which are separated by a larger distance than the measured correlation length. These two specimens may possess less correlated material properties (Einstein, 1983; Villaescusa, 1990; Yu, 1993). 


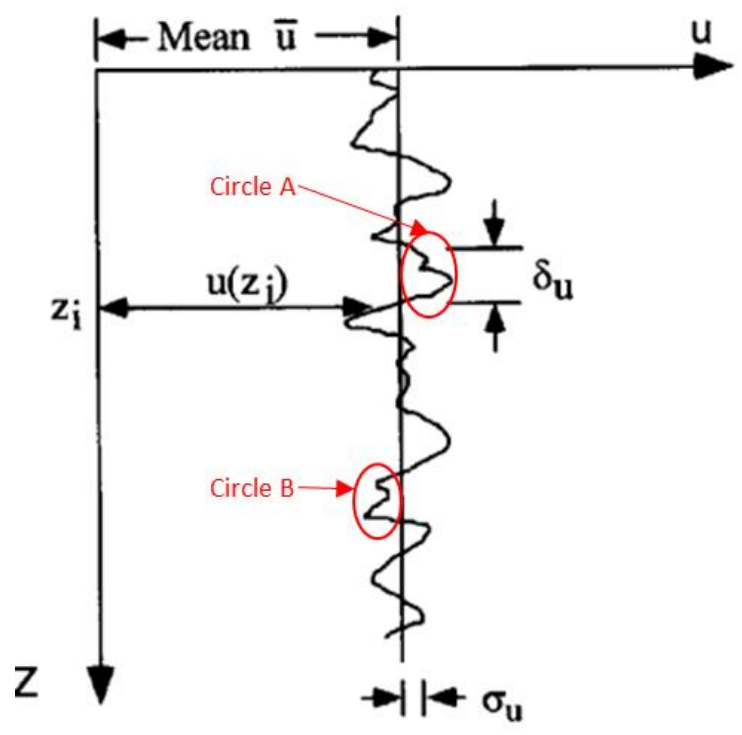

Figure 4.2 Representation of one strength variation at different depths (Vanmarcke, 1977)

Therefore, in order to illustrate the degree of local spatial continuity of rocks and to investigate the influence of the spatial correlation factor on rock behavior, this chapter proposes a method based on the Type III Extreme Value distribution, introduced in Chapter 3, by adding the spatial correlation function.

One form of covariance called a semi-variogram $\gamma(t)$ can define the spatial correlation (Chen, 2012; Ledvina, 1991). The semi-variogram is equal to half of the variance between two random locations separated by distance $t$, as shown in Equation 4.2:

$$
\gamma(t)=\frac{1}{2} \operatorname{Var}[Z(u)-Z(u+t)]
$$

where $Z(u)$ is the random distribution of Gaussian variable at the location $u$. The distance value $t$ is a scalar parameter, including both the spacing value and orientation. It is calculated as shown in Equation 4.3:

$$
\mathrm{t}=\sqrt{\left(\frac{t_{1}}{a}\right)^{2}+\left(\frac{t_{2}}{b}\right)^{2}}
$$


where $t_{1}$ and $t_{2}$ are the spatial interval values along the field's major and minor axes, and $a$ and $b$ are the parameters that show the speed at which the spatial dependence decreases along the two principal axes.

This research assumes that the distribution of the stochastic model is Gaussian. If the initial distribution is non-Gaussian, it is necessary to transfer the distribution to Gaussian distribution. The conversion process will not change the shape of the initial distribution. If the shape of the initial distribution is significantly different from Gaussian distribution, then this approach may not be suitable.

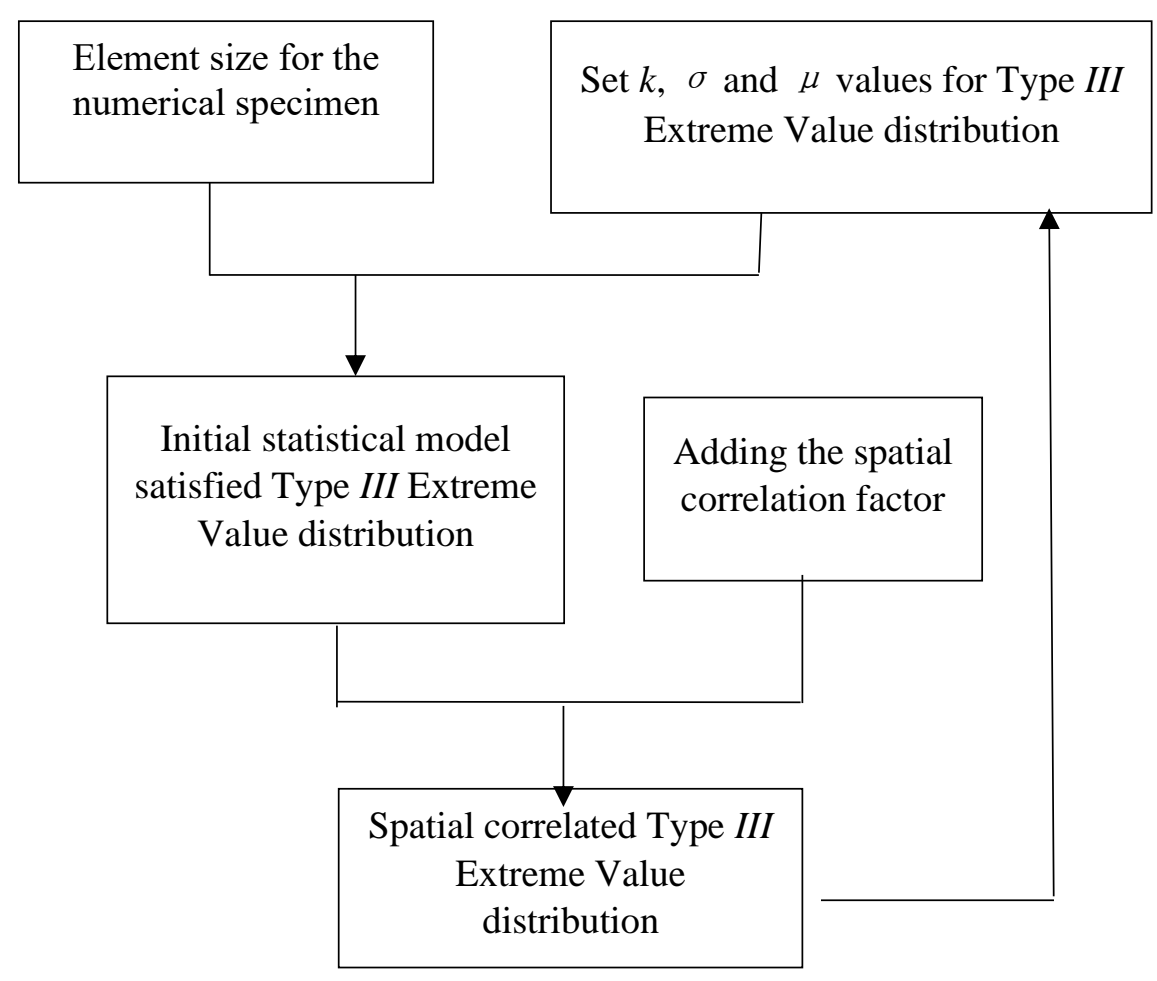

Figure 4.3 Steps to generate the spatial correlated database in MATLAB

The flow chart shown in 
Figure $\mathbf{4 . 3}$ is the demonstration of the data generating process. The initial probabilistic model can be established by one sample which satisfies Type III Extreme Value distribution with given parameters $k, \sigma$ and $\mu$, and the space of the sample includes $N$ numbers, which is the same as the element numbers of our numerical model. Then, based on the characteristics of the intact rock material properties, the spatial correlation is added to generate the objective correlated random field, which will finally satisfy the Type III Extreme Value distribution.

\subsection{Numerical simulation and results discussion}

In this chapter, the commercial geotechnical code FLAC8.0 performed the stochastic numerical simulation. This research used an explicit finite difference method to capture the complex behavior of rock excavation using numerical steps. The models show large displacements and strains and exhibit non-linear material behavior. This includes cases of yield or failure over large areas or total collapse. This simulation used Mohr-Coulomb criterion as the failure criterion where the elements show perfect plastic behavior on the occurrence of failure.

\subsubsection{Model description}

In order to study the influences of randomness and spatial correlation on rock mechanical behavior, this research created three different types of two-dimensional (2D) models (Figure 4.4) which are models with deterministic material, completely random material, and the spatial correlated random model. Here, only cohesion and friction are assumed to satisfy the Type III Extreme Value distribution. Table 4.1 shows the mean values for all the material properties defined in the rock specimens. 
Model dimensions remain constant in the entire modelling effort. Figure 4.4 shows the geometry of the model in 2D. The model is 4 inches in length and 2 inches in width. In addition, the element size is set as 0.2 inch $\times 0.5$ inch.

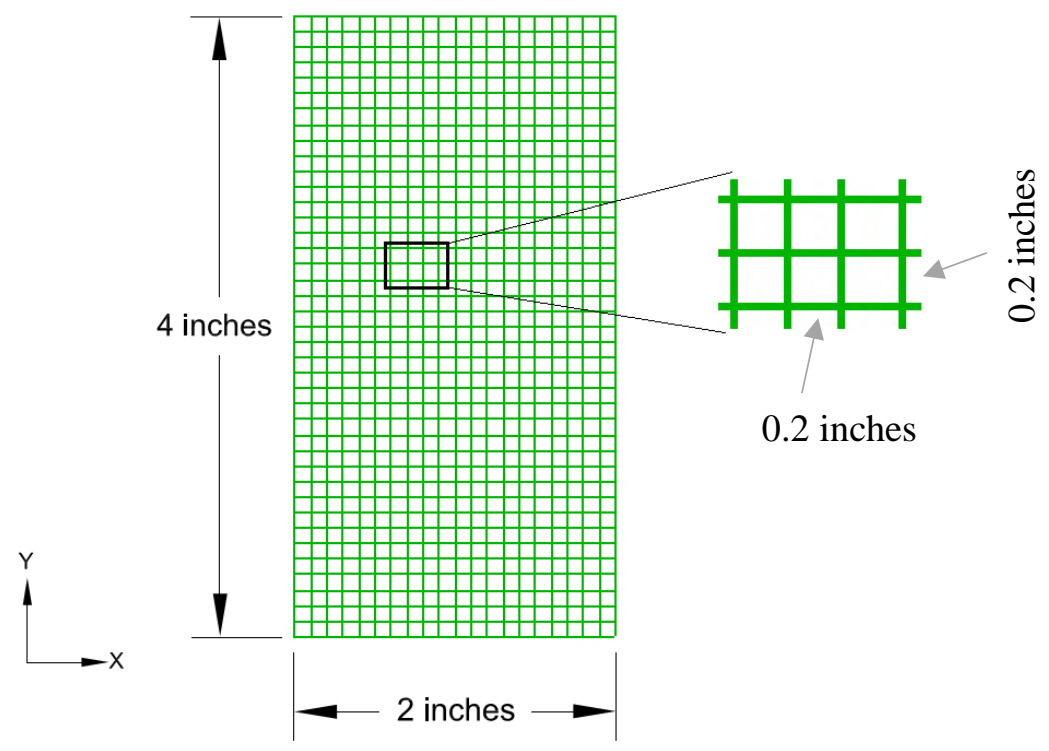

Figure 4.4 The geometry of FLAC8.0 model

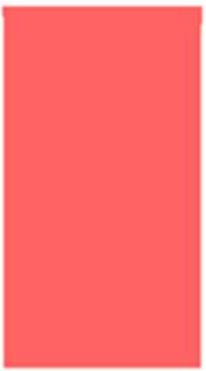

Deterministic model

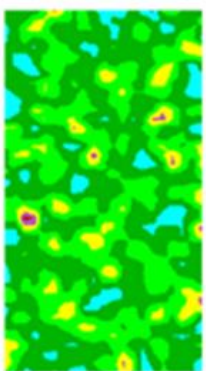

Random model with no spatial correlation

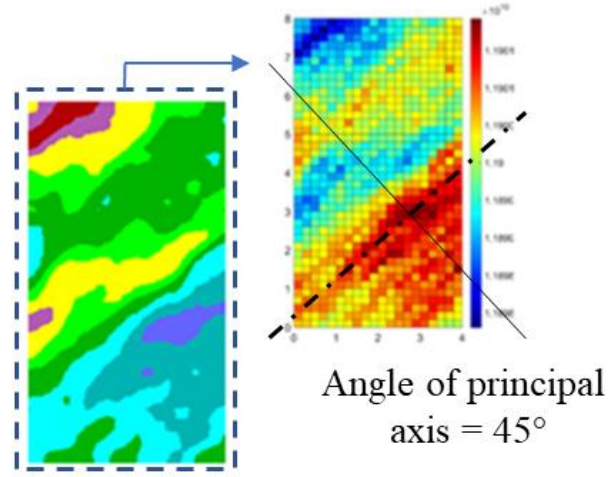

Random model with spatial correlation

Figure 4.5 Material distributions for three representative models

Table 4.1 Mean values for the material properties used in the rock specimens

\begin{tabular}{|c|c|c|c|}
\hline $\begin{array}{c}\text { Bulk modulus } \\
\left(\times \mathbf{1 0}^{6} \mathrm{psi}\right)\end{array}$ & $\begin{array}{c}\text { Shear } \\
\text { modulus } \\
\left(\times \mathbf{1 0}^{6} \mathbf{p s i}\right)\end{array}$ & $\begin{array}{c}\text { Cohesion } \\
(\mathbf{p s i})\end{array}$ & $\begin{array}{c}\text { Friction } \\
(\text { degree })\end{array}$ \\
\hline
\end{tabular}




\begin{tabular}{|l|l|l|l|}
\hline $11,907.62$ & $10,993.88$ & $7,720.37$ & 44 \\
\hline
\end{tabular}

For the spatial correlated random model in Figure 4.5, the angle of the principal axis is set at 45 degrees from the horizontal axis. The principal axis is a line associated with the correlated circle or ellipse, which generates the major and minor axes mentioned in Equation 4.3, with two black dash lines shown in Figure 4.5. The adjacent correlation for the two major axes in this case is strongly different. The spatial dependence reduction is set at 10 inches along the major axis and 2 inches along the minor axis, which means the spatial dependence along the major axis is 5 times larger than along the minor axis.

\subsubsection{Comparison among traditional deterministic model, completely random model, and random model with spatial correlation}

Table 4.2 and Figure 4.6 show the safety factor summaries for each grid for the three models defined in Figure 4.6. The grid number for each model is 800. From the statistical summary in Table 4.2Table 4.2 Summary for safety factors of the three different models , the safety factor for the deterministic varies from 0 to 1.5 ; while for the other two random models, the ranges of the safety factor are from 0 to 5. For the standard deviation, the random model with correlation factor is 0.595 , which is higher than the other two models. The skewness value for the model with correlation factor is 0.9952 , which is close to unity. The shape of the distribution is very similar to the Normal distribution, which matches the rule of large samples in statistical theory that the distribution of one population should behave like a Normal distribution when the number of sample data approaches infinity.

Table 4.2 Summary for safety factors of the three different models

\begin{tabular}{|c|c|c|c|c|c|c|}
\hline \multirow{2}{*}{$\begin{array}{c}\text { Safety } \\
\text { Factor } \\
\text { range }\end{array}$} & \multicolumn{2}{|c|}{ Deterministic } & \multicolumn{2}{c|}{$\begin{array}{c}\text { Random without } \\
\text { correlation }\end{array}$} & \multicolumn{2}{c|}{$\begin{array}{c}\text { Random with } \\
\text { correlation }\end{array}$} \\
\cline { 2 - 7 } & Frequency & Relative & Frequency & Relative & Frequency & Relative \\
\hline
\end{tabular}




\begin{tabular}{|c|c|c|c|c|c|c|}
\hline & & Frequency & & Frequency & & Frequency \\
\hline$<1$ & 759 & 0.95 & 2 & 0.0025 & 0 & 0 \\
\hline $1.0-1.5$ & 41 & 0.05 & 91 & 0.11 & 5 & 0.00623 \\
\hline $1.5-2.0$ & 0 & 0 & 257 & 0.32 & 210 & 0.26 \\
\hline $2.0-2.5$ & 0 & 0 & 253 & 0.32 & 309 & 0.39 \\
\hline $2.5-3.0$ & 0 & 0 & 162 & 0.20 & 142 & 0.18 \\
\hline $3.0-3.5$ & 0 & 0 & 29 & 0.04 & 85 & 0.11 \\
\hline $3.5-4.0$ & 0 & 0 & 4 & 0.005 & 34 & 0.04 \\
\hline $4.0-4.5$ & 0 & 0 & 2 & 0.0025 & 14 & 0.018 \\
\hline $4.5-5.0$ & 0 & 0 & 0 & 0 & 1 & 0.0013 \\
\hline Mean & \multicolumn{2}{|c|}{1.000094} & \multicolumn{2}{|c|}{2.12} & \multicolumn{2}{c|}{2.41} \\
\hline STDV & \multicolumn{2}{|c|}{0.00049} & \multicolumn{2}{|c|}{0.52} & \multicolumn{2}{c|}{0.9952} \\
\hline Skewness & \multicolumn{2}{|c|}{---} &
\end{tabular}

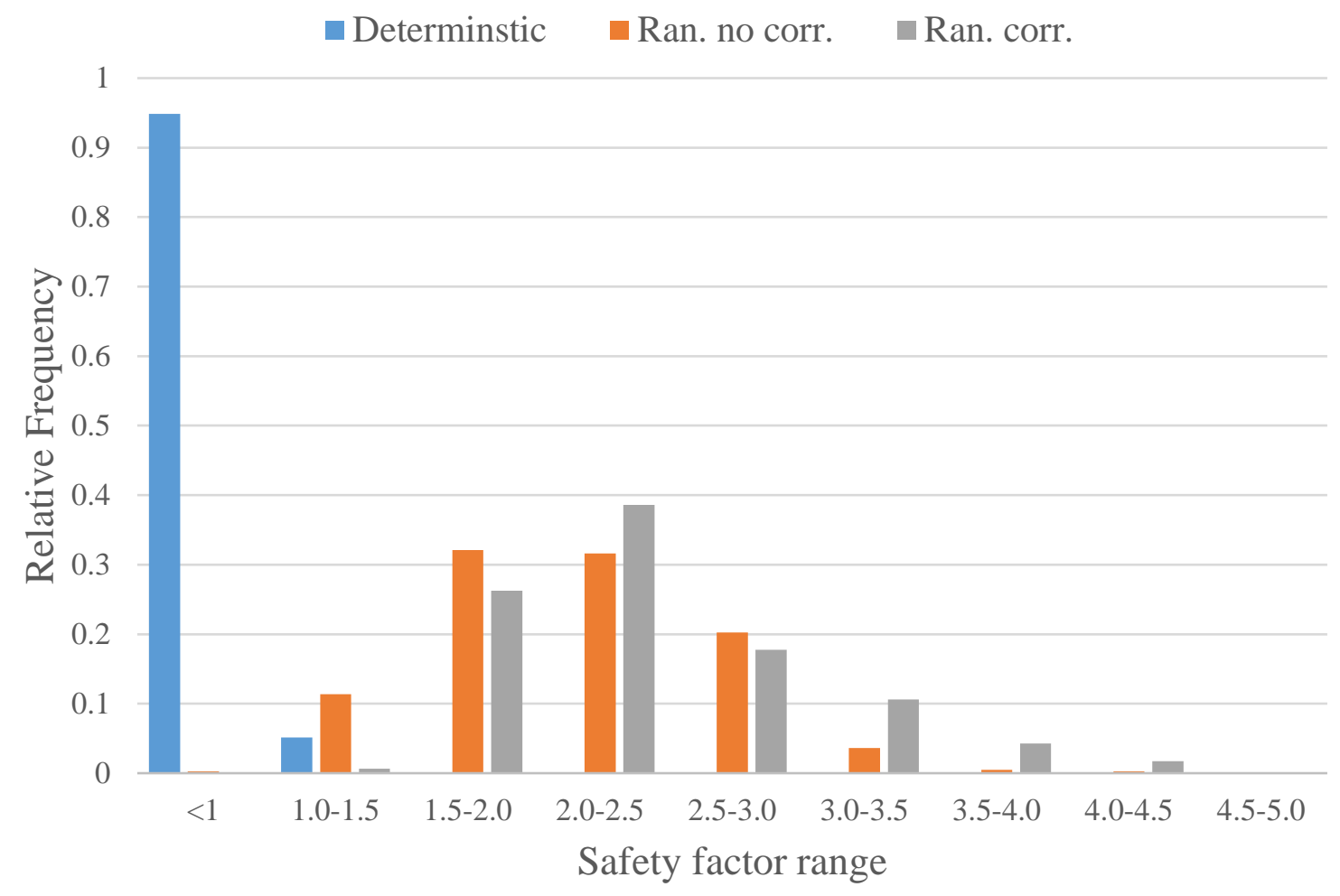

Figure 4.6 Histogram for the safety factor of the three different models

(Note: Ran. no corr.- represents completely random model without spatial correlation.

Ran. corr.- represents random model with spatial correlation.) 
Figure 4.7 shows the stress-strain curves for the three models in Figure 4.5. The peak strengths for models with deterministic material, completely random material and spatial correlated random model are $9.88 \times 10^{3}, 1.32 \times 10^{4}$ and $1.12 \times 10^{4}$ psi respectively. The strength of the spatial correlated random model is in the middle.

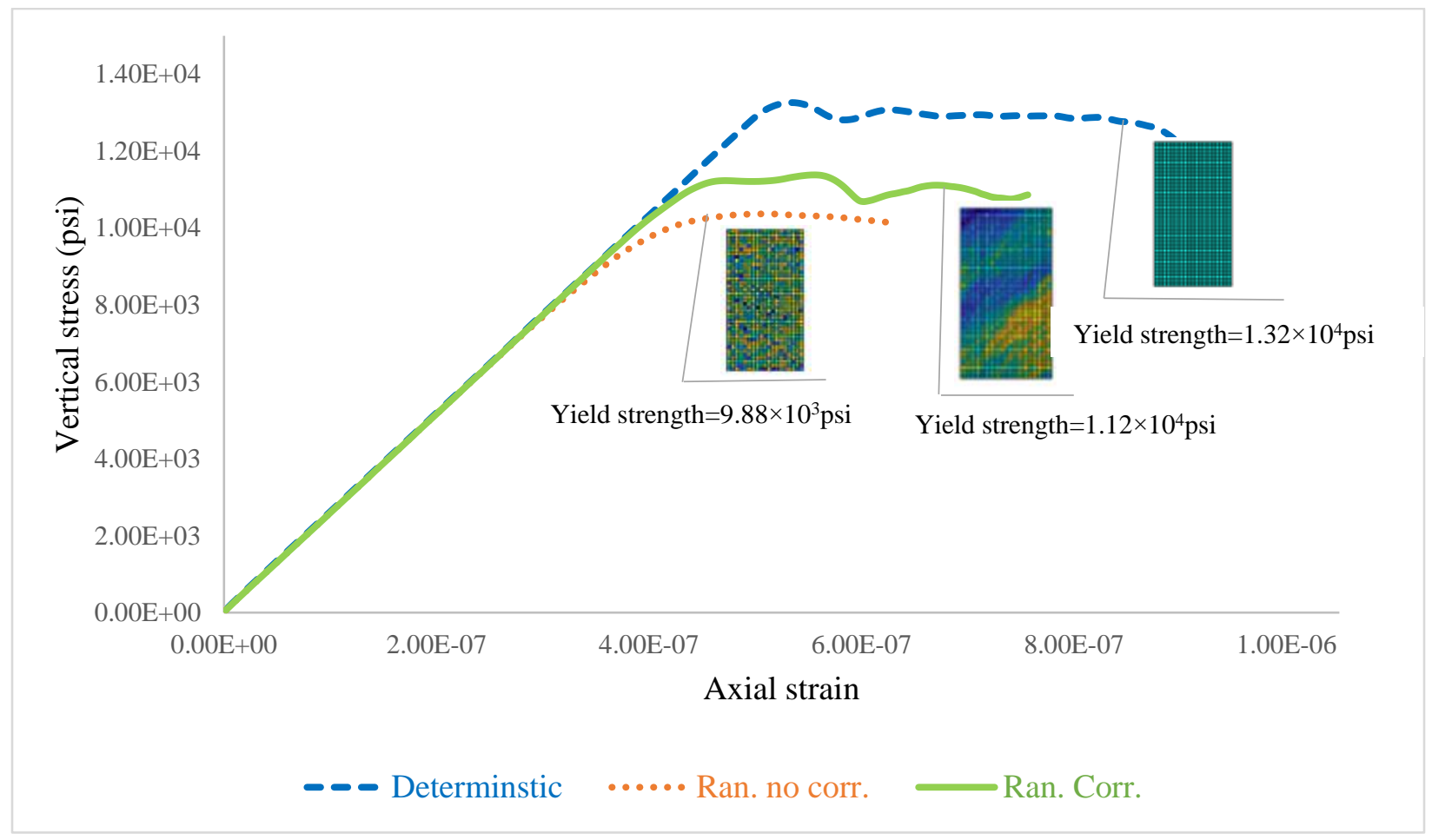

Figure 4.7 Stress-strain curves for the three representative different models

Due to the geology and history of deposition in rock laminations, the properties of the adjacent areas correlate with each other. Therefore, the random model with correlation factor is the closest model to the rockmass. All the above results also verified that the spatial correlated model is the optimal approach to estimate for rockmass strength. The traditionally deterministic method overestimated the strength of the intact rocks, and the completely random model with no correlation underestimated the strength of intact rocks. 


\subsubsection{Results discussion for the random model with spatial correlation factor}

Figure 4.8 shows the vertical stress variations along a vertical cross-section of the specimen (shown in a red dashed line in Figure 4.9). The failed state of the homogeneous rock specimen is shown in Figure 4.9. The same conditions are set for all the specimens except for the spatial dependences (values of $a$ and $b$ in Equation 4.3). The specimens yield around step 3000; therefore, this step is assumed to be the point where the specimens began to fail. Before the yield point, the specimens were still in elastic state, and therefore the distribution of the vertical stress along the central axis is identical. This is independent of the spatial correlation. However, after the yield point, when the specimens enter elastic-plastic state, the distributions of the vertical stress along the central axis are significantly dependent on the spatial correlation.

From Figure 4.9 the contours at the first row represent the correlated materials with different spatial dependence along the two main axes. The dark blue color shows the relatively weak zones, and the yellow color indicates the strong zones. Crack initiations usually occur in weak zones. Further, as the stress redistribution occurs in the specimen, the cracks propagate to the adjacent area. 


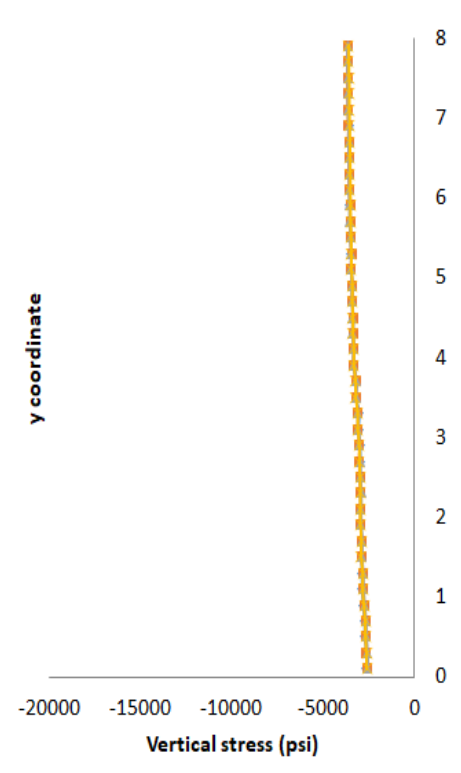

Step 1000

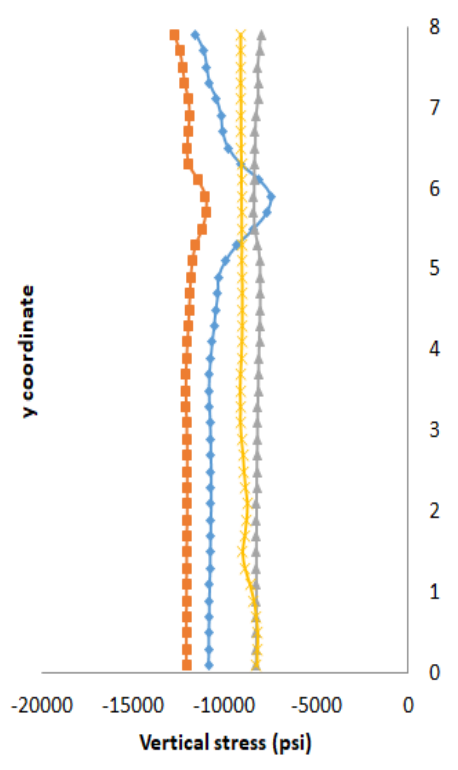

Step 4000

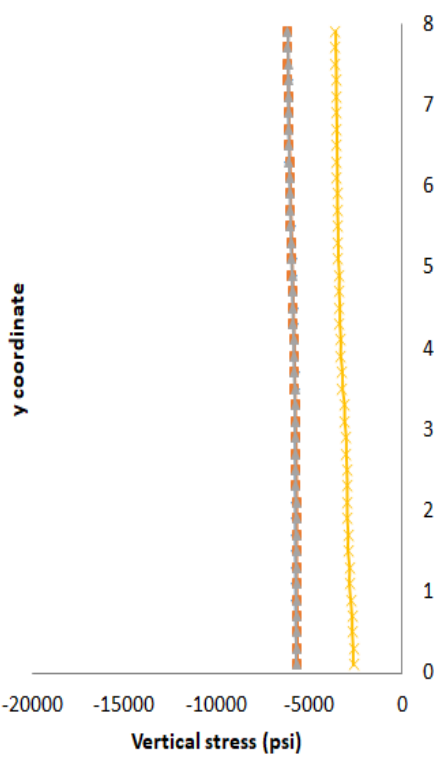

Step 1830

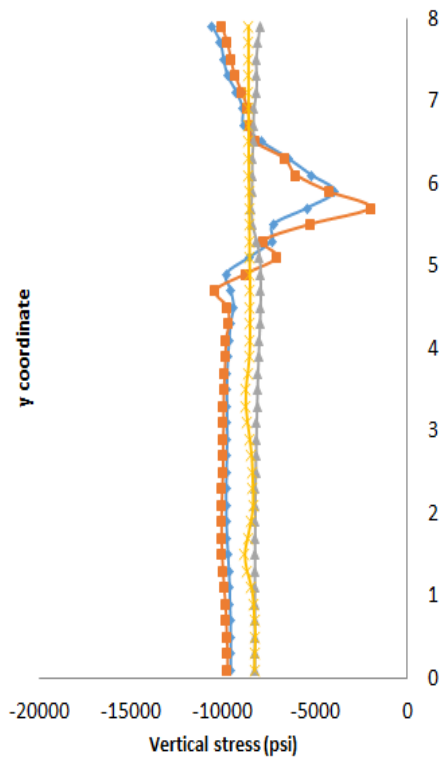

Step 5000

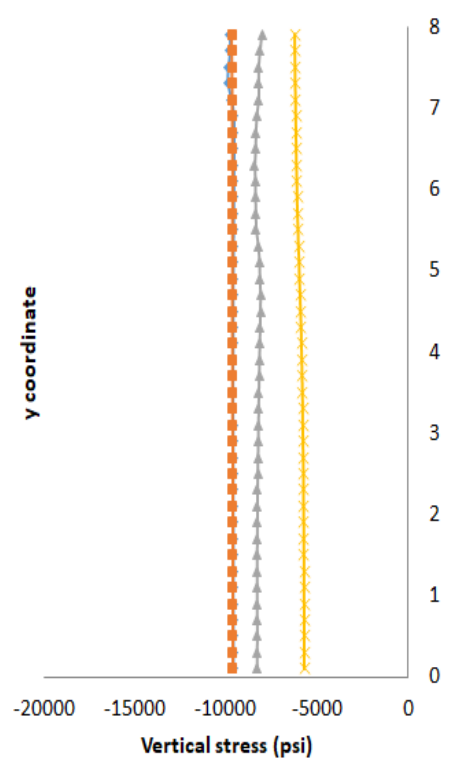

Step 3000

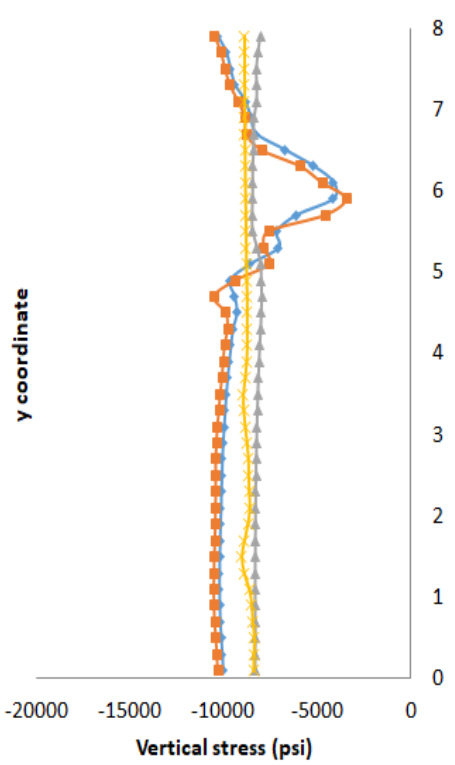

Step 6000

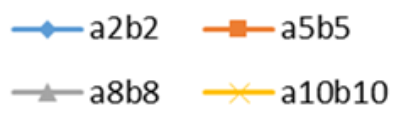

Figure 4.8 Vertical stress variations along the central axes of the specimens

In addition, the spatial correlation is an index of both the correlation and continuity for one random field. As the spatial dependence decreases at a low value, the mechanical behavior of the rock specimen in the adjacent area will correlate less. The change gradient of the mechanical 
response is comparatively larger, and vice versa. Therefore, from Figure 4.8, when $a=2$ and $b=2$, the spatial dependence is relatively low, and the yield state randomness increases with additional run. On the other hand, when the values of $a$ and $b$ are increased, the spatial dependence increases, and the yield states are more stable.

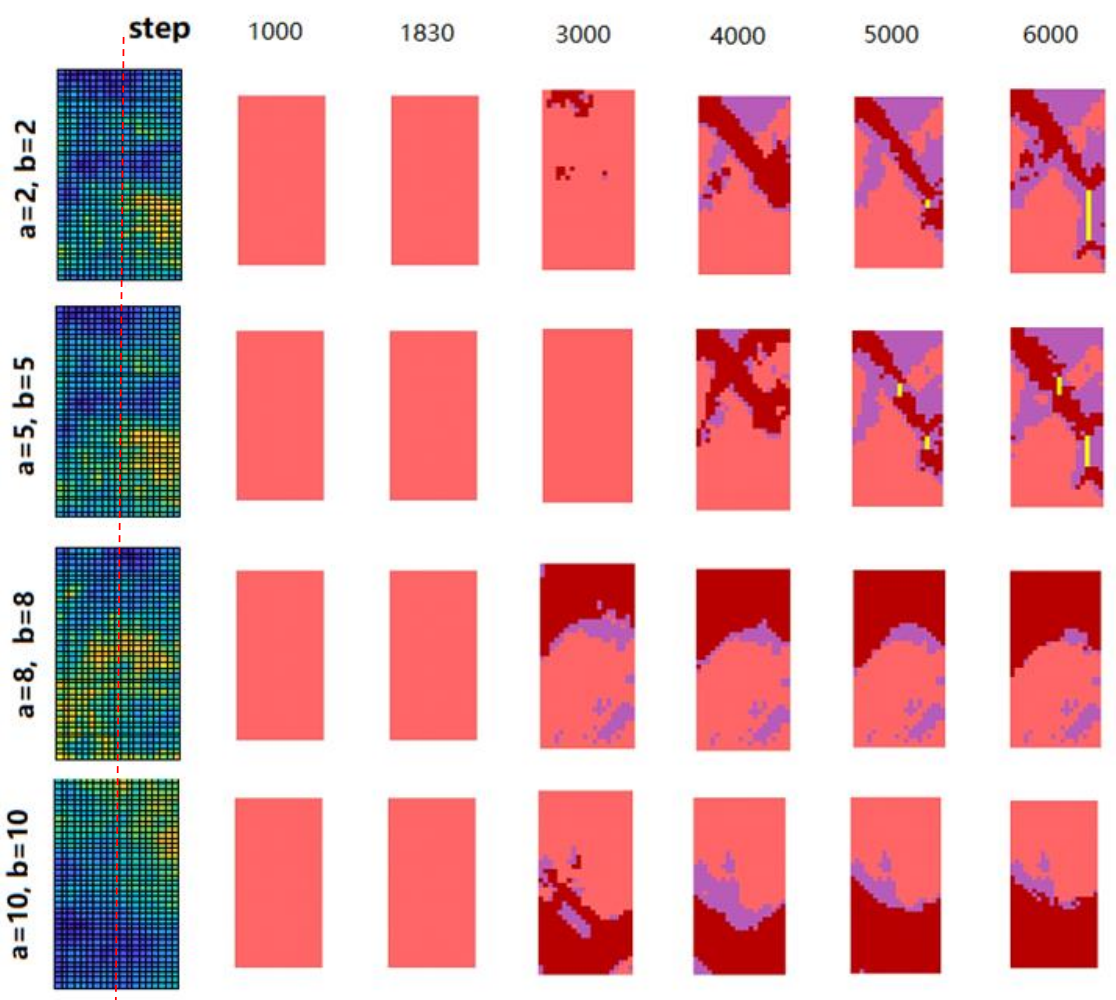

Figure 4.9 Yield state for rock specimen with different spatial dependence

\subsection{Sensitivity analysis for four different parameters:}

\section{friction, cohesion, correlation length along horizontal and vertical axis}

During the research in the previous two sections, in both the complete random model and the spatial correlated random model, randomness slightly influencing the intact rock strength is discovered, and yet significantly influences the final failure mode for rock specimen. The strength of the rock specimen with random material properties is somewhat different from the 
model with deterministic material properties. However, the failure procedure for the model with spatial variance is strongly different from the deterministic model. The micro-cracks are always initiated by the area, which has relatively lower mechanical property values. This phenomenon matches the weakest-link theory concluded by Weibull.

In order to verify the importance of four different parameters (friction, cohesion, and correlation length along horizontal and vertical axes respectively), this research will generate 152 random sample data. The stress for each specimen at different loading steps and different locations were tracked. The aim is to discover how the inherent material parameters affect the internal stress for intact rocks.

Here four different groups for friction's sensitivity analysis are summarized as:

(1) Group One:

Sensitivity analysis for parameter " $b$ " (correlation length along horizontal direction)

a. Constant value: cohesion, mean value of friction, $a / L(a=2,4,6,8)$ Valuable value: " $b$ " (spatial parameter along horizontal direction)

b. Constant value: friction, mean value of cohesion, $a / L(a=2,4,6,8)$ Valuable value: " $b$ " (spatial parameter along horizontal direction)

(2) Group Two:

Sensitivity analysis for parameter " $a$ " (correlation length along vertical direction)

a. Constant value: cohesion, mean value of friction, $b / L(b=4,8,12,16)$ Valuable value: " $a$ " (spatial parameter along vertical direction)

b. Constant value: friction, mean value of cohesion, $b / L(b=4,8,12,16)$ Valuable value: “ $a$ " (spatial parameter along vertical direction)

(3) Group Three: 
Sensitivity analysis for friction

Constant value: cohesion, mean value of friction, " $a, b$ "

Valuable value: standard deviation of friction

$$
\left(C O V_{\varphi}=0.01 / 0.1 / 0.02 / 0.04 / 0.08 / 0.12 / 0.14 / 0.16 / 0.18\right)
$$

(4) Group four:

Sensitivity analysis for cohesion

Constant value: friction, mean value of cohesion, $a, b$

Valuable value: standard deviation of cohesion

$$
\left(\operatorname{COV}_{c}=5 / 10 / 20 / 30 / 40 / 50 / 60 / 70 / 80 / 90\right)
$$

Figure 4.9 clearly shows that the yield state contours, which happened at the failure onset point, always have the same distribution of the friction angle distribution (marked by the red dash rectangle). Therefore, the initial failure for the heterogeneous models may depend on the distribution of the friction initially defined. It was also found that whether it is the complete random model or the spatial correlated random model, the randomness always slightly influences the intact rock strength and significantly influences the final failure mode for rock specimen. The strength of the rock specimen with random material properties is slightly different from the model with deterministic material properties. However, the failure procedure for the model with spatial variance is strongly different from the deterministic model. The micro-cracks are always initiated by the area, which has relatively lower mechanical property values. This phenomenon matches the weakest-link theory concluded by Weibull.

$\begin{array}{llllll}a=2 & a=2 & a=2 & a=4 & a=6 & a=8 \\ b=4 & b=6 & b=8 & b=2 & b=2 & b=2\end{array}$


cohesion

friction

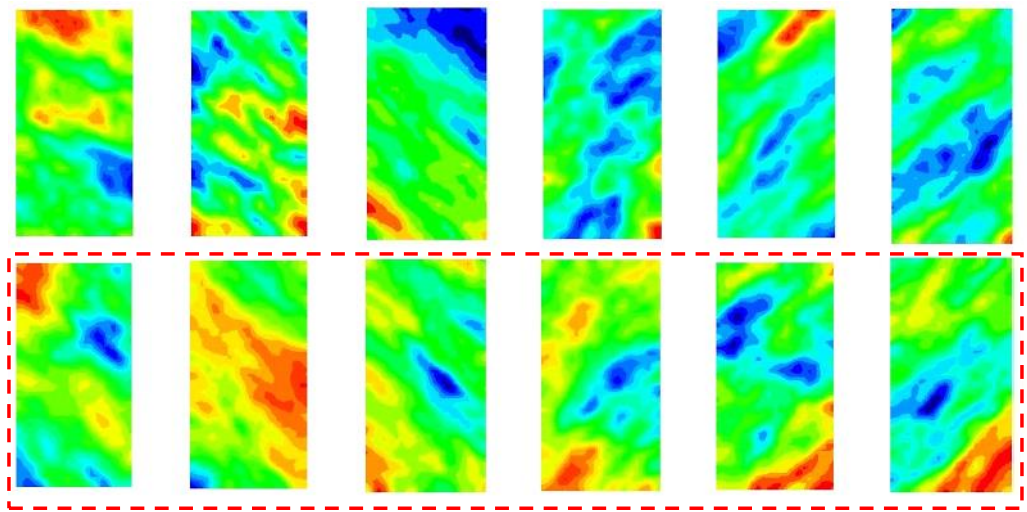

Yield state

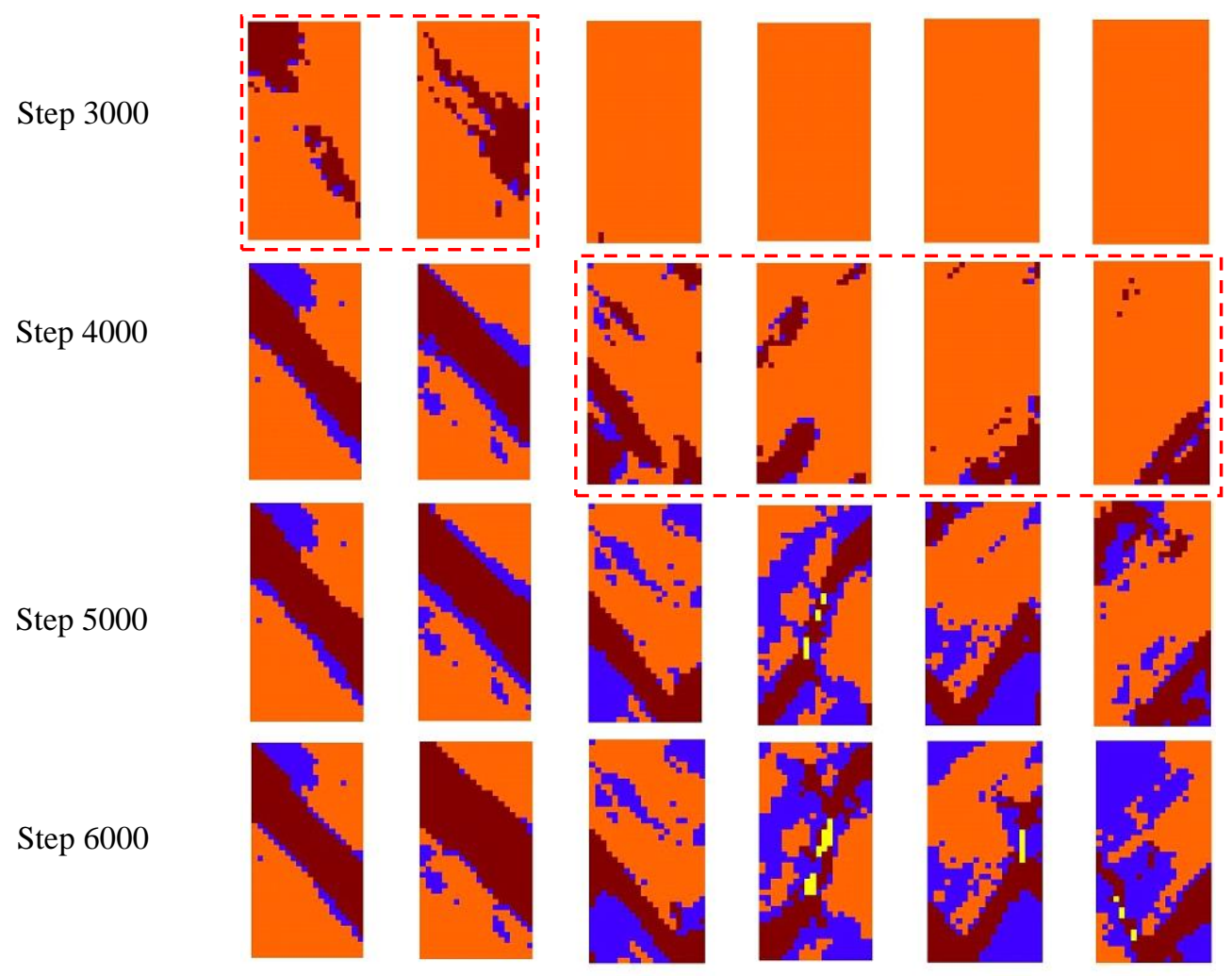

Figure 4.10 Friction, cohesion contours vs. yield state contours with different loading steps

For the sensitivity analysis, as shown in the plots in Figure 4.10, the mean value for the friction angle remains constant in the entire modeling effect this time. Figure 4.10 lists the sensitivity analysis for the friction angle. The constitutive model used here is Mohr-coulomb model.

Here, eight different groups for friction sensitivity analysis are summarized as: 
(1-4) Constant value: cohesion, mean value of friction, $a / L(a=2,4,6,8)$

Valuable value: $b$ (spatial parameter along horizontal direction)

(5-8) Constant value: cohesion, mean value of friction, $b / L(b=4,8,12,16)$

Valuable value: $a$ (spatial parameter along vertical direction)

The geometry and loading condition are kept the same as in the previous analysis. For the spatial correlation definition, two parameters, $a$ and $b$, are used to control correlation length $h$. These two parameters signify how quickly spatial dependence decreases along those axes. When a does not equal $b$, this means the sample is heterogeneous.
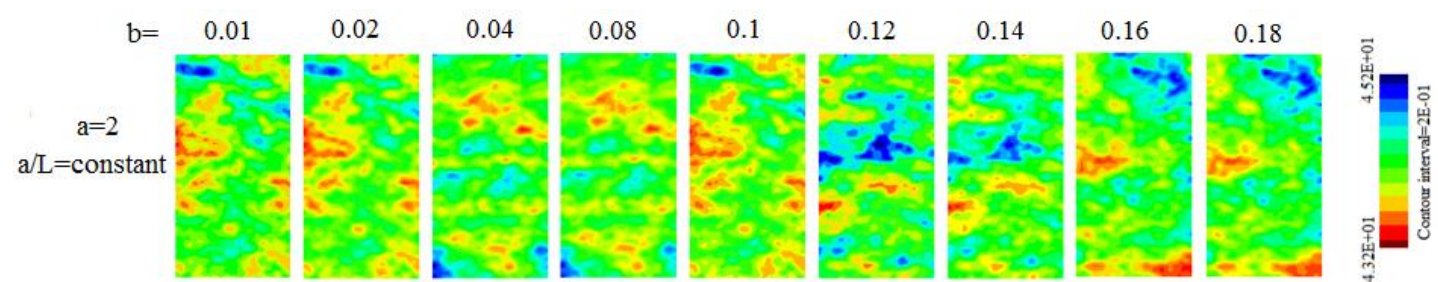

(1-a) friction contours with differenr horizontal correlation length
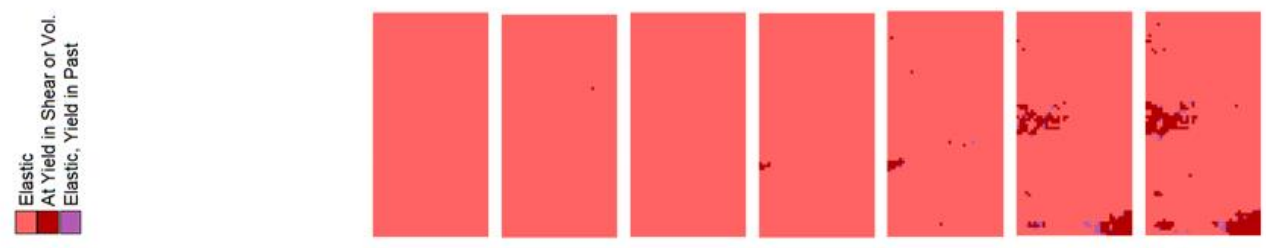

$$
\text { Step }
$$

9000
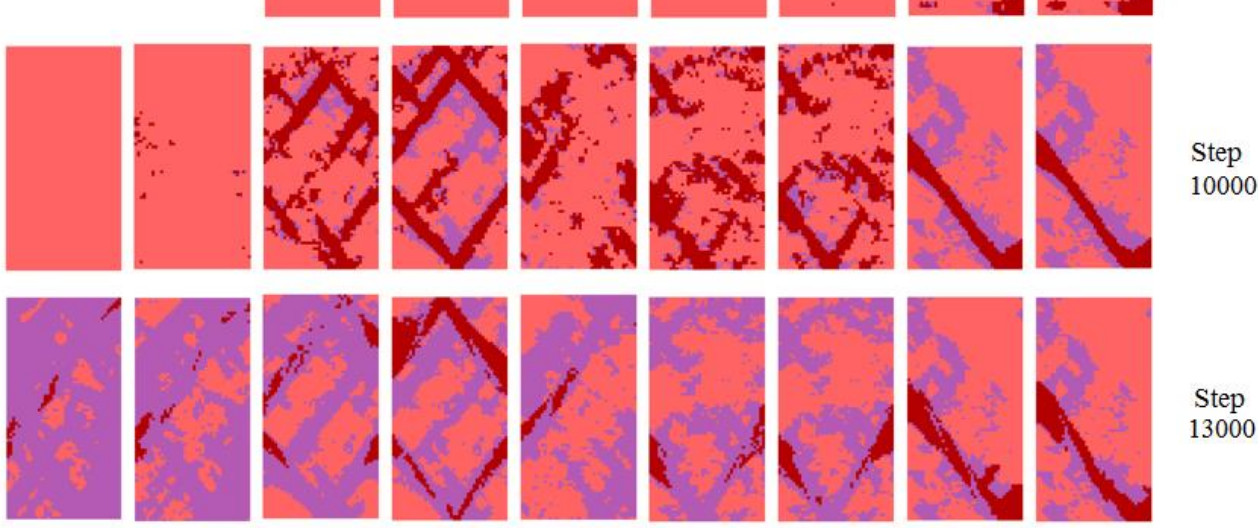

10000

(1-b) failure state at different loading steps

(1) Set cohesion as constant; set friction as a variable value, $a / L(a=2)$ as constant value 


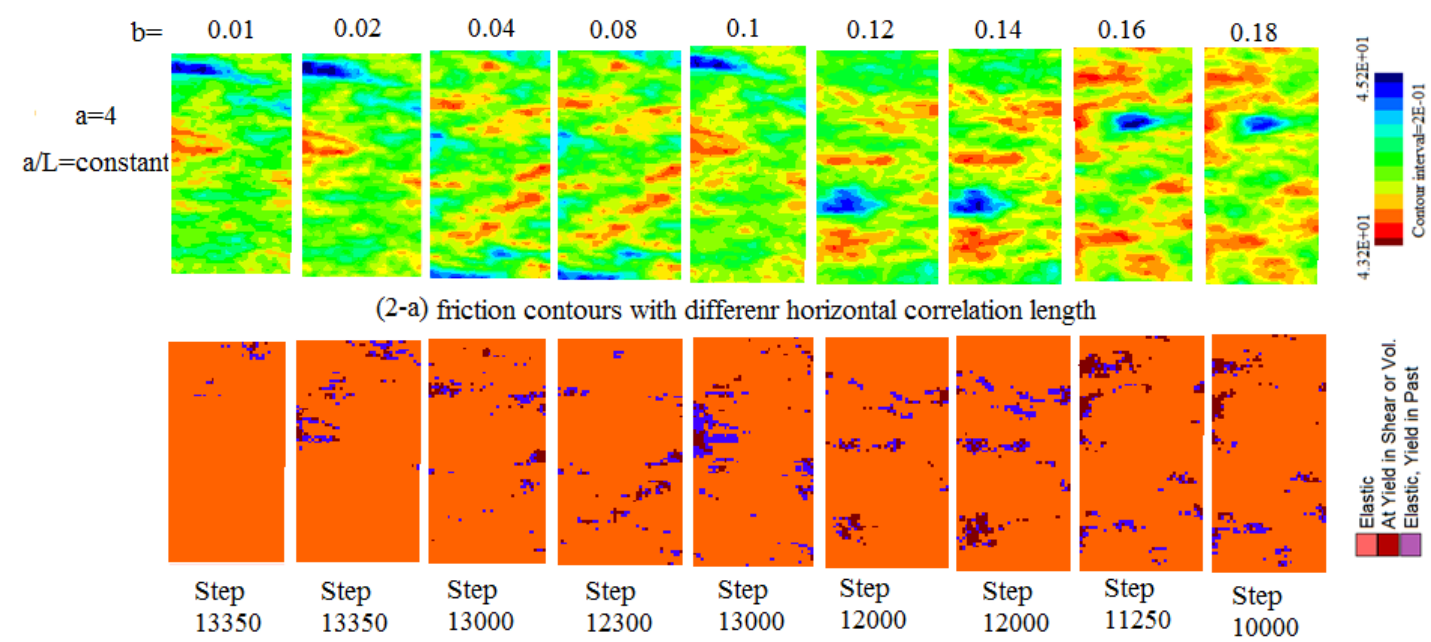

(2-b) failure state at different loading steps

(2) Set cohesion as constant; set friction as a variable value, $a / L(a=4)$ as constant value
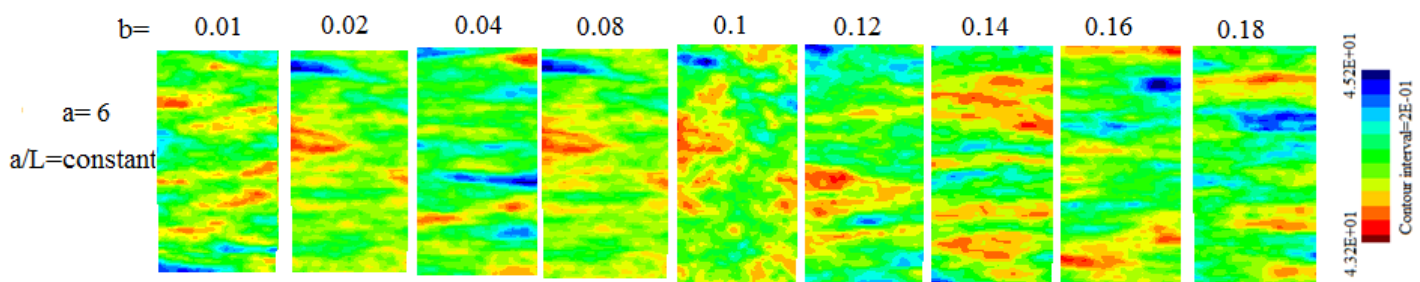

(3-a) friction contours with differenr horizontal correlation length
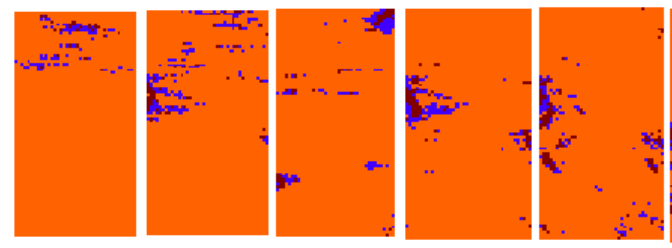

13300
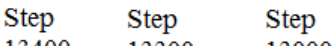

$13000 \quad 12600$
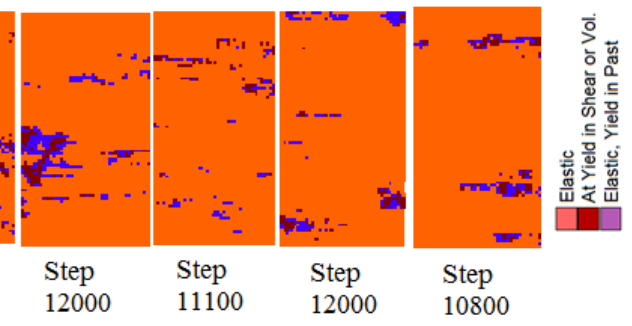

(3-b) failure state at different loading steps

(3) Set cohesion as constant; set friction as a variable value, $a / L(a=6)$ as constant value 


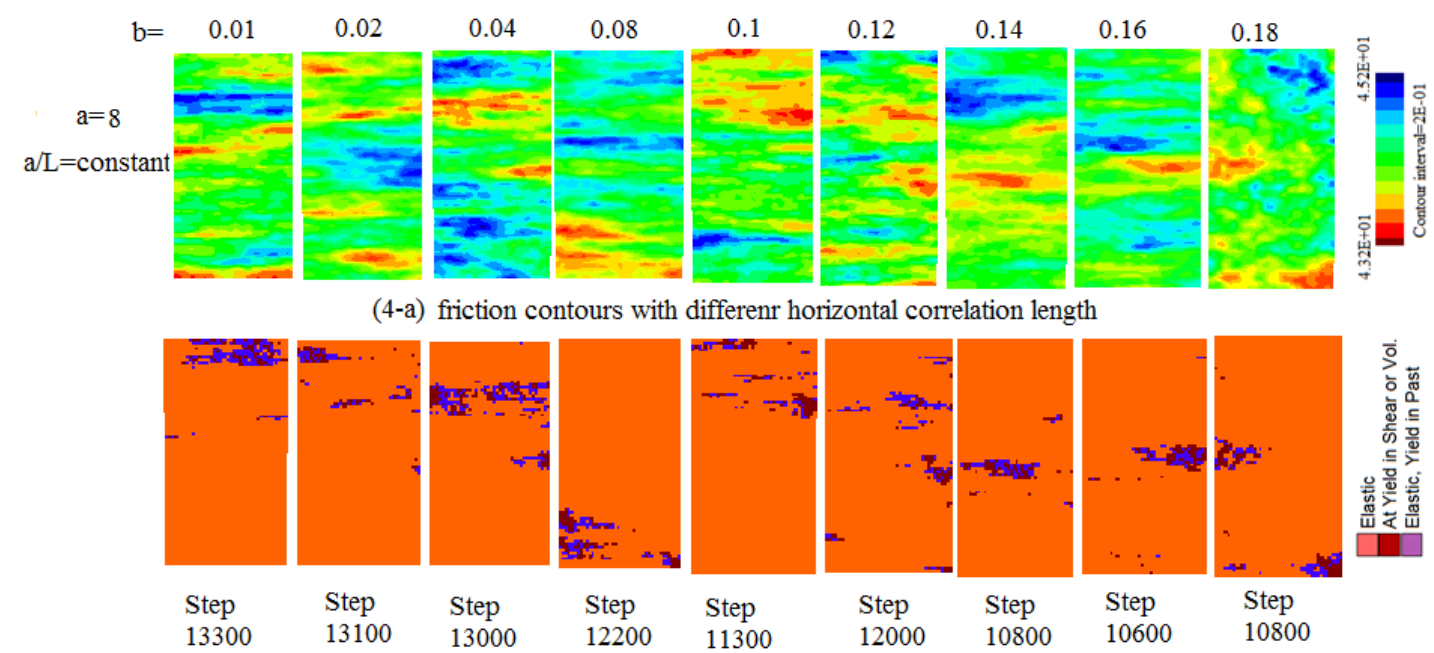

(4-b) failure state at different loading steps

(4) Set cohesion as constant; set friction as a variable value, $a / L(a=8)$ as constant value

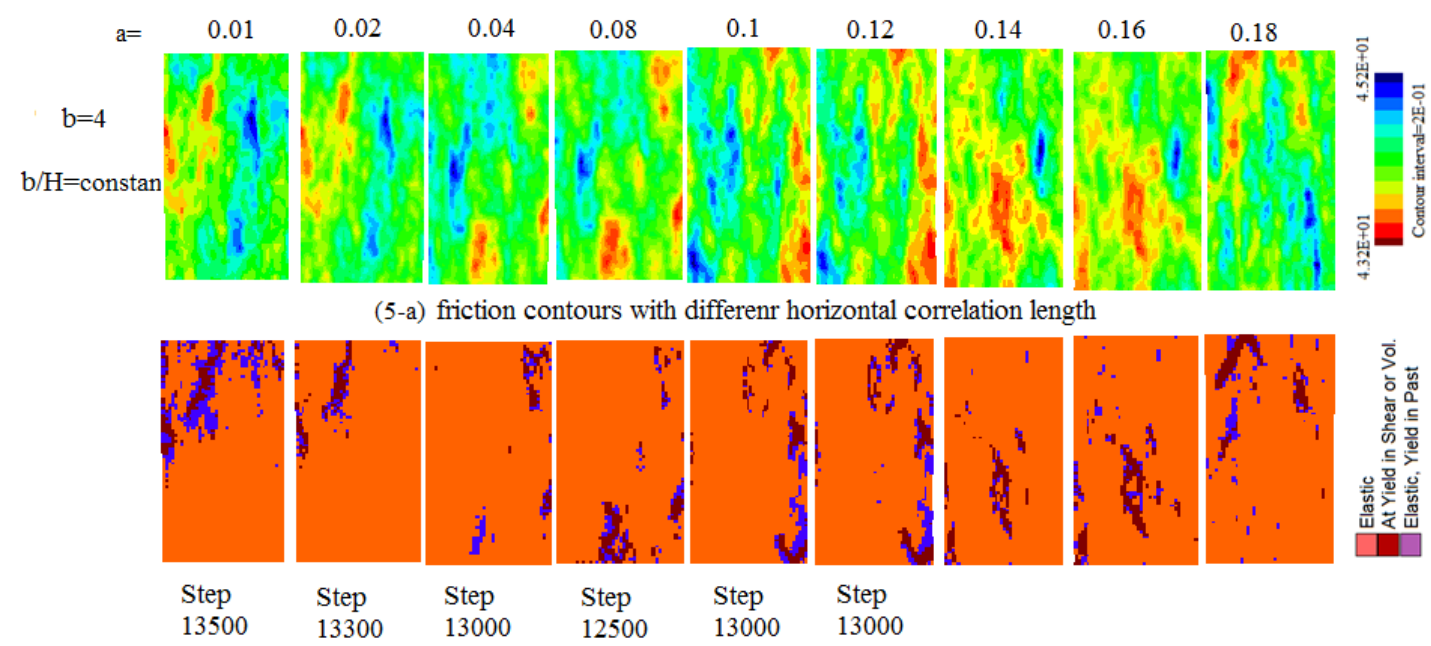

(5-b) failure state at different loading steps

(5) Set cohesion as constant; set friction as a variable value, $b / H(b=4)$ as constant value 


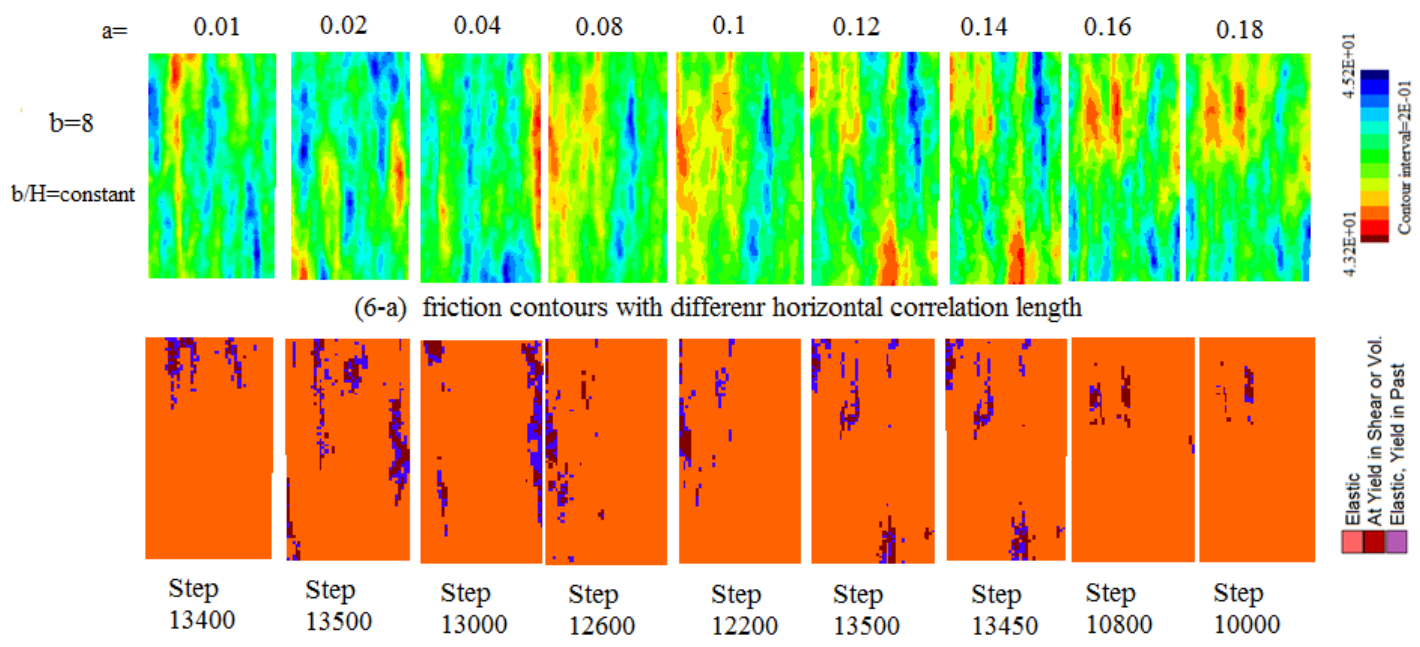

(6-b) failure state at different loading steps

(6) Set cohesion as constant; set friction as a variable value, $b / H(b=8)$ as constant value

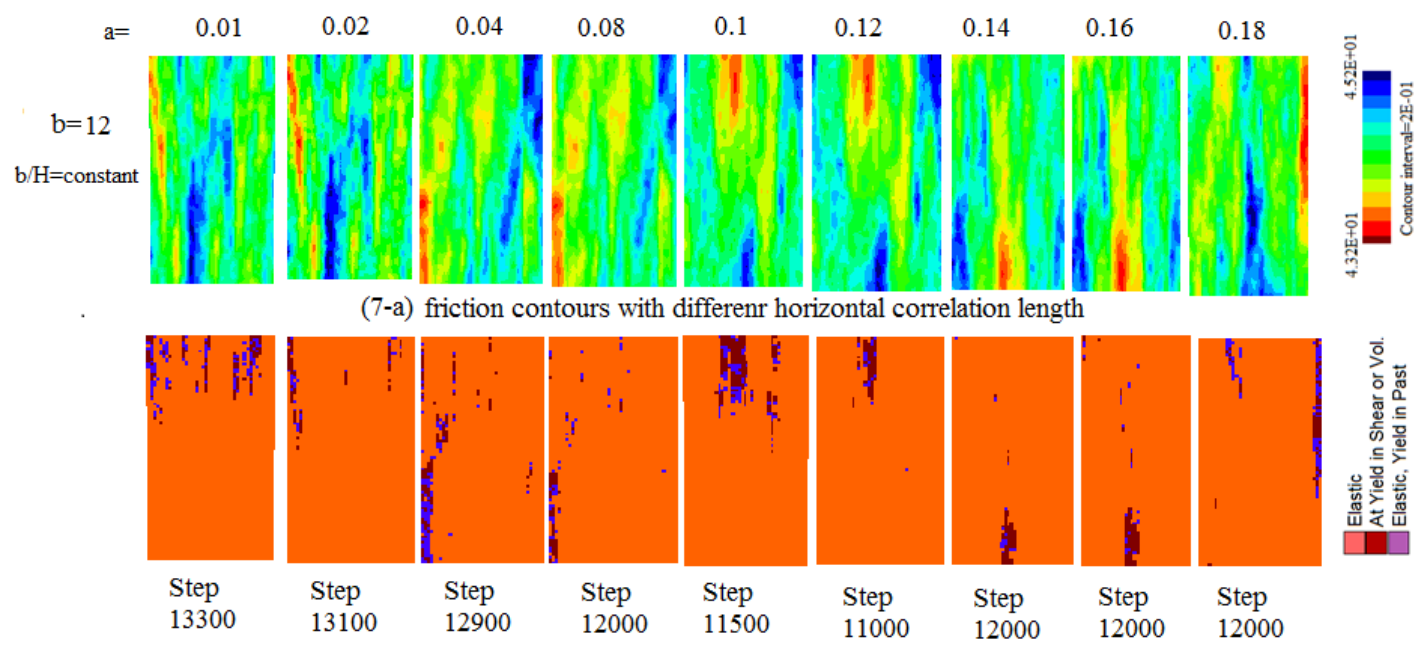

(7-b) failure state at different loading steps

(7) Set cohesion as constant; set friction as a variable value, $b / H(a=12)$ as constant value 


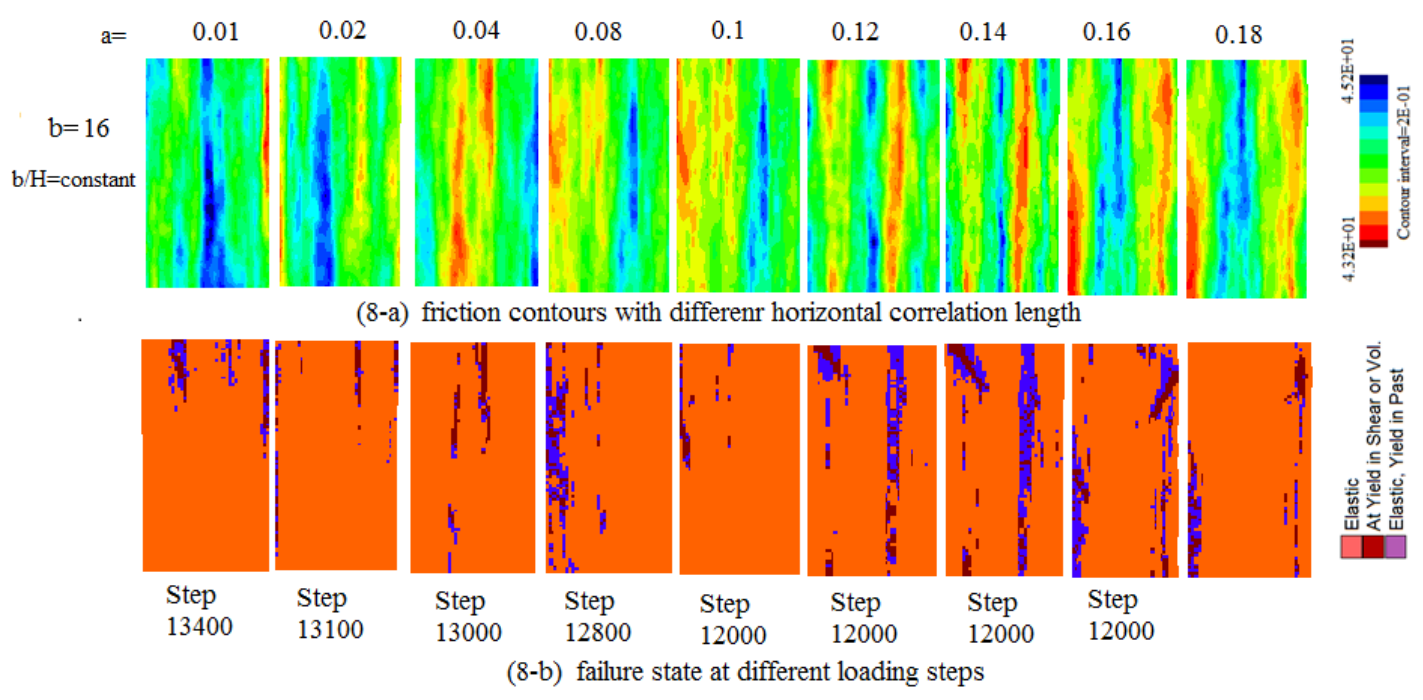

(8) Set cohesion as constant; set friction as a variable value, $b / H(a=16)$ as constant value

Figure 4.11 Sensitivity analysis for the friction angle parameter

From Figure 4.11, the state failure represents the initial damage that occurred in the sample as load was applied and incremented in steps. It is observable that the yield state contours, which happened at the failure onset point, always have the same distribution of the friction angle distribution. The initial failure for the heterogeneous models may depend on the distribution of the friction initially defined. All the distributions of the failure zone for each sample that happened after the yield point are almost the same as the distribution of the friction defined previously. This may represent that the friction angle is the primary factor causing damage to the rock sample. After that, the spatial correlated friction angle combined with the spatial correlated cohesion value to influence the following failure process.

\subsection{Conclusions}

The focus of the research in this chapter was to demonstrate that spatial correlation significantly affects rock strength and failure propagation. Conclusions are summarized as follows: 
Based on one type of stochastic model, this research improved the conventional Type III Extreme Value distribution by adding the spatial correlation factor. The spatial correlation revealed both the correlation and continuity that exists inherently in rock. The numerical simulation results show that the spatial correlated random model can estimate the rock strength in a relatively more realistic way.

For the spatially correlated random model, the small cracks normally initiate at the relatively weak zones. As the spatial dependence increases, the failure state of the rock specimen changes from random to a relatively stable state.

Friction angle is the primary factor that causes damage to the rock sample. After initial failure happens, the spatial correlated friction angle will combine with spatial correlated cohesion value to influence the following failure propagations.

These results show the significant influence of the spatial correlation on the strength and mechanical response of rock. 


\section{CHAPTER 5 \\ CALIBRATION STUDY THROUGH TRIAXIAL TEST}

\subsection{Introduction}

In order to have confidence in the previous model results, some form of calibration of the model with the Hoek-Cell triaxial laboratory test must be significant. The test involves confining a cylindrical sandstone specimen in a pressurized cell, which can simulate a stress condition, and then shearing to failure. In order to determine the shear strength properties of the sample, this research performs triaxial tests for each specimen under different confinement conditions.

This research performed the sandstone triaxial testing using the Hoek Cell, designed to determine the triaxial strength of diamond drill cores of rock. The first step is to fill a stainlesssteel chamber with oil for the application of confining pressure through a rubber-sealing sleeve. Axial and radial strain measurements are taken by using electric strain gauges glued directly onto the cylindrical specimen. At the end of the assembly, the cell on the compression machine platen for cohesion and internal friction determination will be placed.

Experiments can establish the real spatial variance distribution; on the other hand, based on the mechanical parameters obtained from the laboratory, the method proposed above are used to reproduce the material distribution of the intact rock block through MATLAB. Finally, the whole rock block model was created by using FLAC8.0, applying the material properties generated in MATLAB on the whole block. This method will have the specimens be randomly excavated from the large block, which have the same locations as in the laboratory.

The main objective of this chapter is to check the strength difference between laboratory test and numerical simulation. If the results match each other as expected then the model results 
obtained from the numerical models are acceptable, verifying that the proposed stochastic method is appropriate for this research.

\subsection{Comparison between laboratory tests and numerical simulations}

\subsubsection{Model design for Hoek-cell triaxial laboratory tests}

The laboratory test in this chapter is based on a typical triaxial test; this process involves confining a cylindrical sandstone specimen in a pressurized cell, which will simulate a stress condition, and then shearing to failure. In order to determine the shear strength properties of the sample, this research performed the triaxial tests for each specimen under different confinement conditions.

The test was conducted on high-quality undisturbed sandstone specimens. Fifty specimens were drilled from one same large rock block, shown in Figure 5.1.
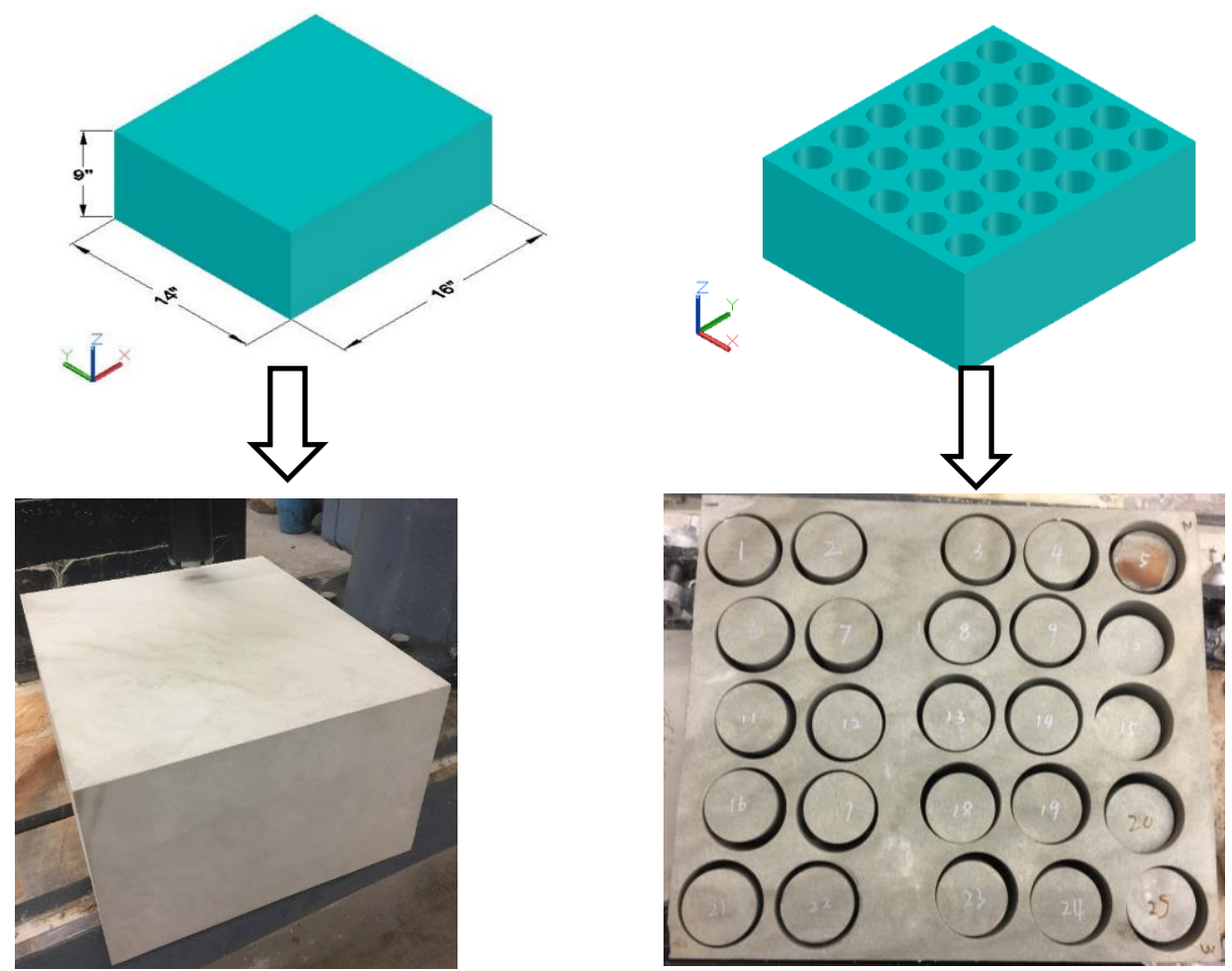

Figure 5.1 Sandstone block before and after drilling 
The dimensions for the whole block are 14" for width, 16" for length, and 9" for height. The height to diameter ratio for the all the rock specimens is 2 (Height $=4$ inches, Diameter=2 inches).

Here, tests ran on two samples for each drill hole (shown in Figure 5.1) under different confining pressures, which determined the parameters as follows:

1) The strength and elastic properties.

2) Shear strength at different confining pressures.

3) Angle of shearing resistance and cohesion.

Because of the nonlinearity of center coordinates, AutoCAD were used to capture all the coordinates of the center for each drill hole, as listed in Table 5.1. The cohesion and friction values for all the specimens were then calculated from the laboratory tests. 
Table 5.1 Summary of center coordinates for each drill hole

\begin{tabular}{|c|c|c|c|c|c|c|c|c|c|c|}
\hline \multirow{2}{*}{ Number } & \multicolumn{2}{|c|}{1} & \multicolumn{2}{|c|}{2} & \multicolumn{2}{|c|}{3} & \multicolumn{2}{|c|}{4} & \multicolumn{2}{|c|}{5} \\
\hline & $1-1$ & $1-2$ & $2-1$ & $2-2$ & $3-1$ & $3-2$ & $4-1$ & $4-2$ & $5-1$ & $5-2$ \\
\hline $\mathrm{X}$ & \multicolumn{2}{|c|}{1.1316} & \multicolumn{2}{|c|}{2.8366} & \multicolumn{2}{|c|}{5.813} & \multicolumn{2}{|c|}{7.3834} & \multicolumn{2}{|c|}{9.1184} \\
\hline $\mathrm{Y}$ & \multicolumn{2}{|c|}{8.2815} & \multicolumn{2}{|c|}{8.401} & \multicolumn{2}{|c|}{8.476} & \multicolumn{2}{|c|}{8.4608} & \multicolumn{2}{|c|}{8.3263} \\
\hline \multirow{2}{*}{ Number } & \multicolumn{2}{|c|}{6} & \multicolumn{2}{|c|}{7} & \multicolumn{2}{|c|}{8} & \multicolumn{2}{|c|}{9} & \multicolumn{2}{|c|}{10} \\
\hline & 6-1 & $6-2$ & $7-1$ & $7-2$ & $8-1$ & $8-2$ & $9-1$ & $9-2$ & $10-1$ & $10-2$ \\
\hline $\mathrm{X}$ & \multicolumn{2}{|c|}{1.4407} & \multicolumn{2}{|c|}{3.2105} & \multicolumn{2}{|c|}{5.5587} & \multicolumn{2}{|c|}{7.2488} & \multicolumn{2}{|c|}{8.9538} \\
\hline $\mathrm{Y}$ & \multicolumn{2}{|c|}{6.6673} & \multicolumn{2}{|c|}{6.727} & \multicolumn{2}{|c|}{6.8317} & \multicolumn{2}{|c|}{6.8915} & \multicolumn{2}{|c|}{6.6374} \\
\hline \multirow{2}{*}{ Number } & \multicolumn{2}{|c|}{11} & & & & & & & & \\
\hline & $11-1$ & $11-2$ & $12-1$ & $12-2$ & $13-1$ & $13-2$ & $14-1$ & $14-2$ & $15-1$ & $15-2$ \\
\hline$X$ & & & & & & & & & & \\
\hline Y & & & & & & & & & & \\
\hline & & & & & & & & & & \\
\hline & $16-1$ & $16-2$ & $17-1$ & $17-2$ & $18-1$ & $18-2$ & $19-1$ & $19-2$ & $20-1$ & $20-2$ \\
\hline $\mathrm{X}$ & & & & & & & & & & \\
\hline $\mathrm{Y}$ & & & & & & & & & & \\
\hline & & & & & & & & & & \\
\hline & $21-1$ & $21-2$ & $22-1$ & $22-2$ & $23-1$ & $23-2$ & $24-1$ & $24-2$ & $25-1$ & $25-2$ \\
\hline $\mathrm{X}$ & & & & & & & & & & \\
\hline $\mathrm{Y}$ & & & & & & & & & & \\
\hline
\end{tabular}

After obtaining these 25 cylindrical sandstone specimens, we confirmed that the height of each specimen is 4 inches and the diameter is 2 inches. As the ratio requirement of height and diameter for Hoek-cell triaxial test is two, the separation of each cylindrical sandstone into two small rock specimens is necessary. 
The triaxial cell consists of a hollow steel cylinder with threaded removable ends, shown in Figure 5.2 (a). A urethane rubber sleeve incorporating U-shaped seals to form a pressurization chamber for the hydraulic fluid is mounted within the cell. Inside the cell is a quick-connect inlet and an outlet with a plug for saturation of the pressurization chamber. At each end of the cell is a spherical seat, which will apply an axial load to the flattened ends of the sample. After grinding the rock specimens flat and saturating the pressurization chamber, the cylindrical rock sample into the chamber within the confining membrane was inserted. Figure 5.2(b) shows the two spherical seats, which are positioned so that the rock core lies centrally in the triaxial chamber.

After applying a small confining pressure to hold the rock core in place, the cell with its spherical seats in a loading frame was placed and a small axial load to hold the system was applied firmly in place. The triaxial tests ran after an adjustment of the confining pressure to the required value. Figure 5.2(c) shows two of the confining pressure conditions for each drill hole through the machine.

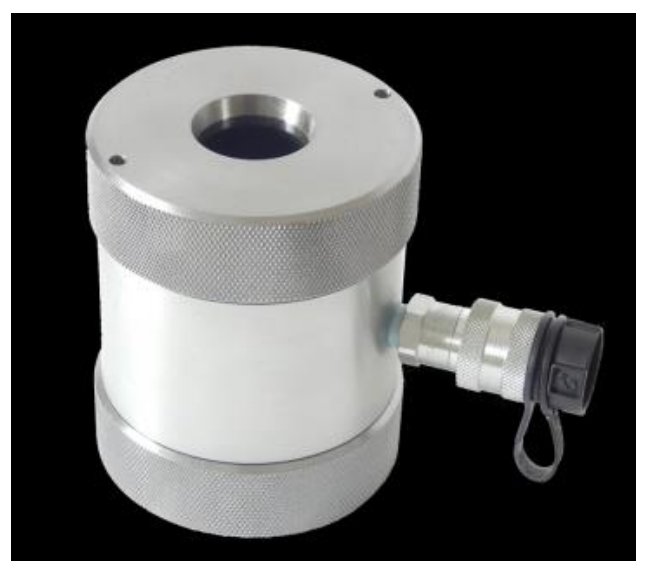

(a) 


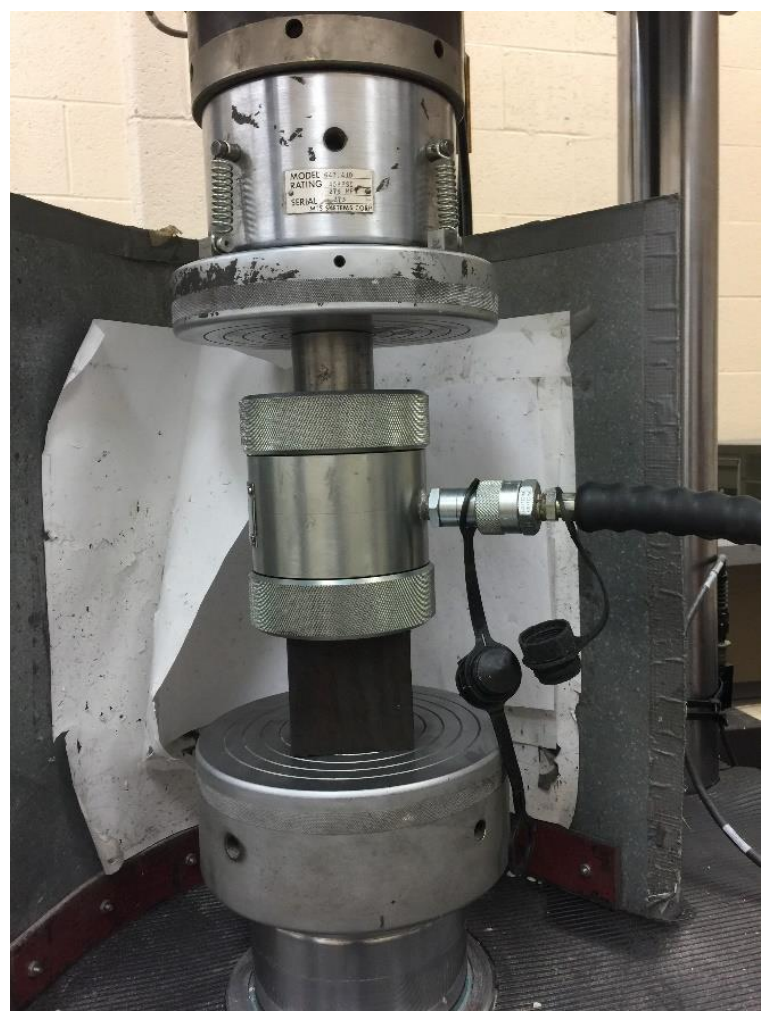

(b)

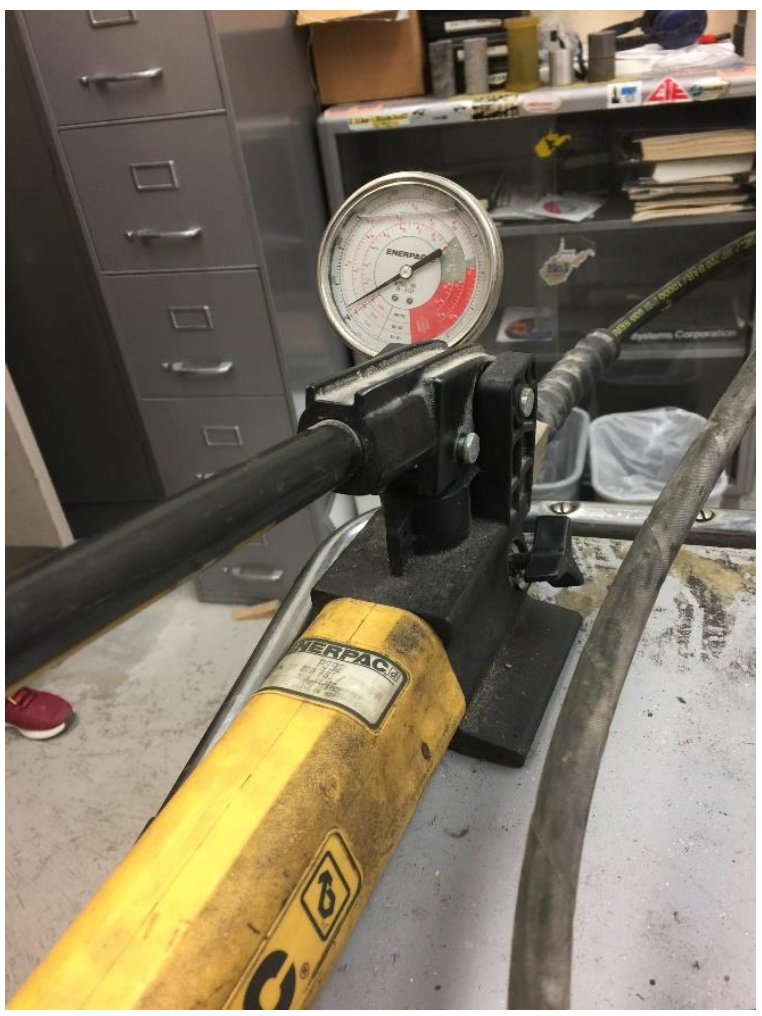

(c)

Figure 5.2 Schematic diagram for the Hoek-cell Triaxial experimental equipment

This experiment considers two different confining pressure conditions for each drill hole, which is 200 psi for the specimen in the above layer and 500 psi for the specimen in the bottom layer. Table 5.2 contains the summary of minimum principal stresses for each specimen. After knowing the minimum and maximum principal stresses for each drill hole, the friction and cohesion values is calculated through RocLab (Rocscience, 2020). 
Table 5.2 Summary of minimum principal stress for each specimen under different confining pressures (Unit: psi)

\begin{tabular}{|c|c|c|c|c|c|c|c|c|c|c|}
\hline \multirow{2}{*}{ Number } & \multicolumn{2}{|c|}{1} & \multicolumn{2}{|c|}{2} & \multicolumn{2}{|c|}{3} & \multicolumn{2}{|c|}{4} & \multicolumn{2}{|c|}{5} \\
\hline & $1-1$ & $1-2$ & $2-1$ & $2-2$ & $3-1$ & $3-2$ & $4-1$ & $4-2$ & $5-1$ & $5-2$ \\
\hline$\sigma_{3}$ & 200 & 500 & 200 & 500 & 200 & 500 & 200 & 500 & 200 & 500 \\
\hline$\sigma_{l}$ & 13,637 & 16,848 & 13,033 & 16,086 & 13,804 & 17,081 & 12,604 & 14,388 & 12,112 & 15,042 \\
\hline \multirow{2}{*}{ Number } & \multicolumn{2}{|c|}{6} & \multicolumn{2}{|c|}{7} & \multicolumn{2}{|c|}{8} & \multicolumn{2}{|c|}{9} & \multicolumn{2}{|c|}{10} \\
\hline & $6-1$ & $6-2$ & $7-1$ & $7-2$ & $8-1$ & $8-2$ & $9-1$ & $9-2$ & $10-1$ & $10-2$ \\
\hline$\sigma_{3}$ & 200 & 500 & 200 & 500 & 200 & 500 & 200 & 500 & 200 & 500 \\
\hline$\sigma_{l}$ & 13,748 & 17,088 & 13,318 & 16,517 & 12,993 & 16,007 & 13,459 & 15,743 & 13,147 & 15,319 \\
\hline \multirow{2}{*}{ Number } & \multicolumn{2}{|c|}{11} & \multicolumn{2}{|c|}{12} & \multicolumn{2}{|c|}{13} & \multicolumn{2}{|c|}{14} & \multicolumn{2}{|c|}{15} \\
\hline & $11-1$ & $11-2$ & $12-1$ & $12-2$ & $13-1$ & $13-2$ & $14-1$ & $14-2$ & $15-1$ & $15-2$ \\
\hline$\sigma_{3}$ & 200 & 500 & 200 & 500 & 200 & 500 & 200 & 500 & 200 & 500 \\
\hline$\sigma_{l}$ & 12,946 & 16,938 & 13,358 & 15,401 & 12,692 & 15,181 & 14,613 & 16,977 & 13,542 & 17,201 \\
\hline \multirow{2}{*}{ Number } & \multicolumn{2}{|c|}{16} & \multicolumn{2}{|c|}{17} & \multicolumn{2}{|c|}{18} & \multicolumn{2}{|c|}{19} & \multicolumn{2}{|c|}{20} \\
\hline & $16-1$ & $16-2$ & $17-1$ & $17-2$ & $18-1$ & $18-2$ & $19-1$ & $19-2$ & $20-1$ & $20-2$ \\
\hline$\sigma_{3}$ & 200 & 500 & 200 & 500 & 200 & 500 & 200 & 500 & 200 & 500 \\
\hline$\sigma_{l}$ & 14,372 & 14,913 & 13,843 & 15,953 & 14,256 & 18,110 & 13,338 & 14,734 & 13,288 & 13,448 \\
\hline \multirow{2}{*}{ Number } & \multicolumn{2}{|c|}{21} & \multicolumn{2}{|c|}{22} & \multicolumn{2}{|c|}{23} & \multicolumn{2}{|c|}{24} & \multicolumn{2}{|c|}{25} \\
\hline & $21-1$ & $21-2$ & $22-1$ & $22-2$ & 23-1 & $23-2$ & 24-1 & $24-2$ & $25-1$ & $25-2$ \\
\hline$\sigma_{3}$ & 200 & 500 & 200 & 500 & 200 & 500 & 200 & 500 & 200 & 500 \\
\hline$\sigma_{l}$ & 14,382 & 15,894 & 13,034 & 16,557 & 13,350 & 17,032 & 14,642 & 17,519 & 14,561 & 18,550 \\
\hline
\end{tabular}

\subsubsection{Model descriptions for numerical simulations}

Due to the computing limitation of the codes, which generated the spatial correlated random database, only two-dimensional numerical simulation was considered for the large rock block calibration. Numerical model was developed using a finite difference software, FLAC8.0. As 
shown in Figure 5.3, the dimensions of the simulation were 140 inches $\times 160$ inches with a total of 22,400 zones.

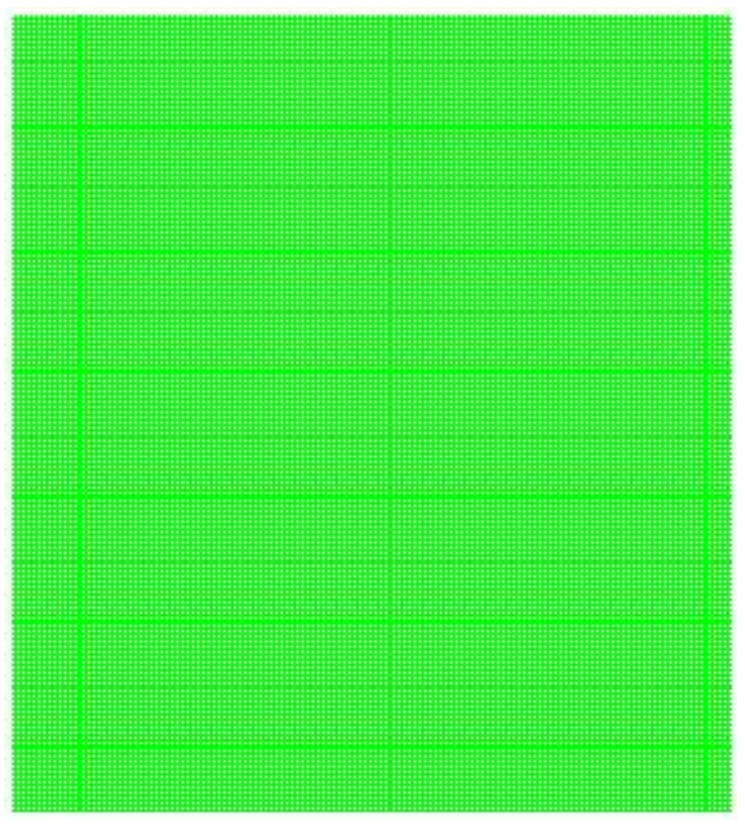

Figure 5.3 Schematic diagram of the large rock block created in FLAC8.0

Table 5.3 lists the laboratory results of the cohesion and friction angle values located in twenty-five different locations in the large rock block. Upon knowing the information listed in the table, the correlation length from both horizontal and vertical directions can be calculated through the methodology introduced in Chapter 4. Based on these two parameters, MATLAB can reveal the inherent material property inside the rock block realistically. Here, we assumed there are no discontinuities existing in the rock block. The spatial variance's existence is only due to the material property. 
Table 5.3 Summary about friction angle and cohesion values for each specimen

\begin{tabular}{|c|c|c|c|c|c|c|c|c|c|}
\hline Number & $\mathbf{1}$ & $\mathbf{2}$ & $\mathbf{3}$ & $\mathbf{4}$ & $\mathbf{5}$ & $\mathbf{6}$ & $\mathbf{7}$ & $\mathbf{8}$ & $\mathbf{9}$ \\
\hline $\begin{array}{c}\text { Cohesion } \\
(\mathrm{psi})\end{array}$ & 736.36 & 692.70 & 749.4 & 587.55 & 631.50 & 747.96 & 715.91 & 688.79 & 675.15 \\
\hline $\begin{array}{c}\text { Friction } \\
\left(^{\circ}\right)\end{array}$ & 38.82 & 38.3 & 39.05 & 31.71 & 38 & 39.31 & 38.85 & 38.14 & 34.57 \\
\hline \hline Number & $\mathbf{1 0}$ & $\mathbf{1 1}$ & $\mathbf{1 2}$ & $\mathbf{1 3}$ & $\mathbf{1 4}$ & $\mathbf{1 5}$ & $\mathbf{1 6}$ & $\mathbf{1 7}$ & $\mathbf{1 8}$ \\
\hline $\begin{array}{c}\text { Cohesion } \\
(\mathrm{psi})\end{array}$ & 649.48 & 705.75 & 650.1 & 646 & 745.2 & 742.9 & 421.3 & 681.68 & 795.1 \\
\hline $\begin{array}{c}\text { Friction } \\
\left(^{\circ}\right)\end{array}$ & 34.01 & 42.03 & 33.24 & 35.7 & 34.84 & 40.59 & 16.72 & 33.57 & 41.1 \\
\hline $\mathbf{N u m b e r}^{\text {Number }}$ & $\mathbf{1 9}$ & $\mathbf{2 0}$ & $\mathbf{2 1}$ & $\mathbf{2 2}$ & $\mathbf{2 3}$ & $\mathbf{2 4}$ & $\mathbf{2 5}$ & & \\
\hline $\begin{array}{c}\text { Cohesion } \\
(\mathrm{psi})\end{array}$ & 577.3 & 359.3 & 641.79 & 706.34 & 730 & 782.5 & 818.6 & & \\
\hline $\begin{array}{c}\text { Friction } \\
\left.{ }^{\circ}\right)\end{array}$ & 28.73 & 13.67 & 29.6 & 40.22 & 40.7 & 37.23 & 41.49 & & \\
\hline
\end{tabular}

Due to the computational resource limitation and the fact that the code used to generate the random data is only suitable for the two-dimensional model, here only the cohesion and friction angle were set in spatially correlated random condition to be in the two-dimensional condition. Figure 5.4 shows a realization of a random field for both cohesion and friction. 

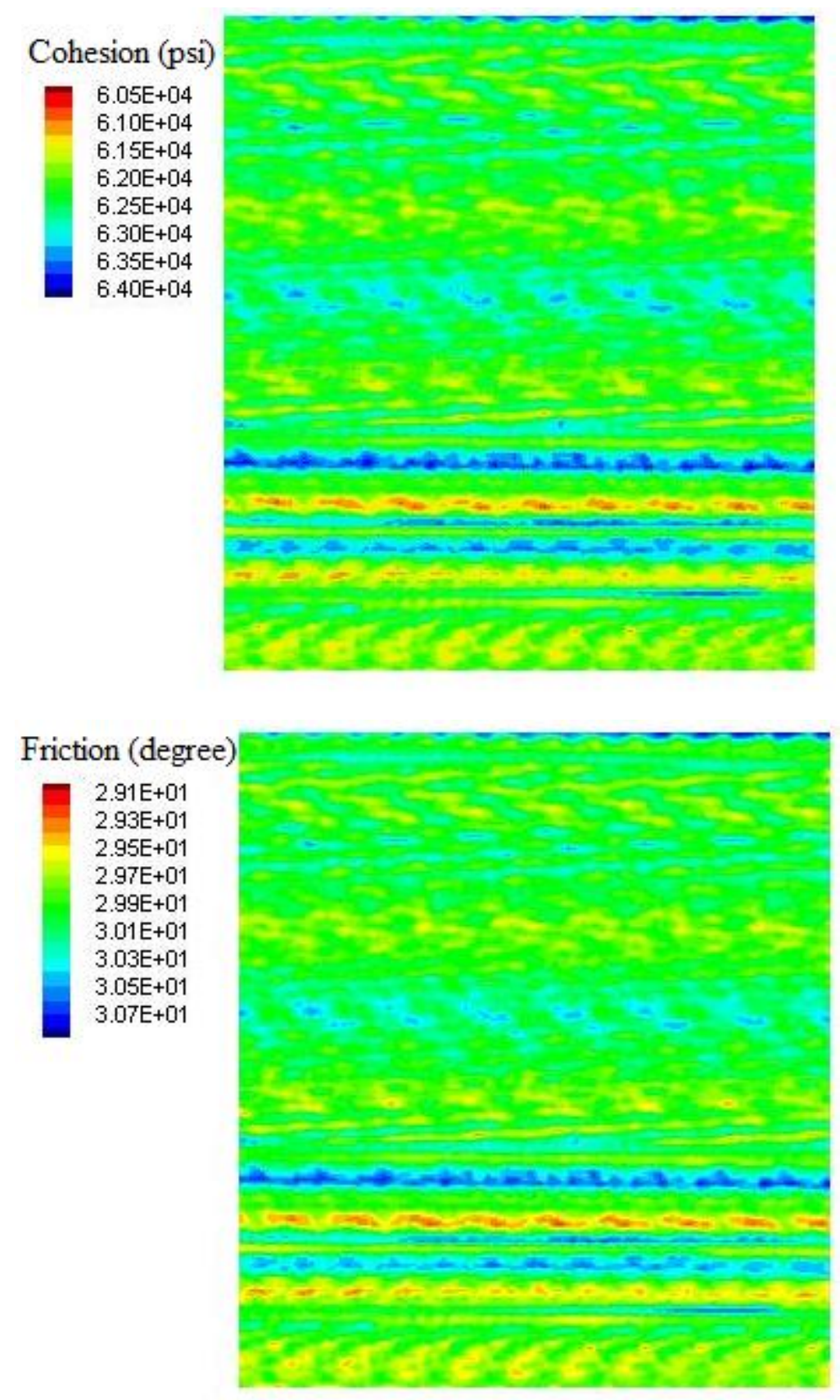

Figure 5.4 Random field's distributions for two parameters: cohesion and friction angle

Then the triaxial compression test on the specimens was performed. First, they selected specimens randomly from the large rock block assigned with random parameters generated with correlation length. Areas were selected within the large rock block to extract specimens, such as specimens with diameters of 2 inches and 4 inches, shown in Figure 5.5. Fourteen specimens 
were tested with specimens of 2 inches in diameter and 4 inches in length. Based on the cohesion contour and the specimen locations, the specimens were selected with different mechanical properties from the block.

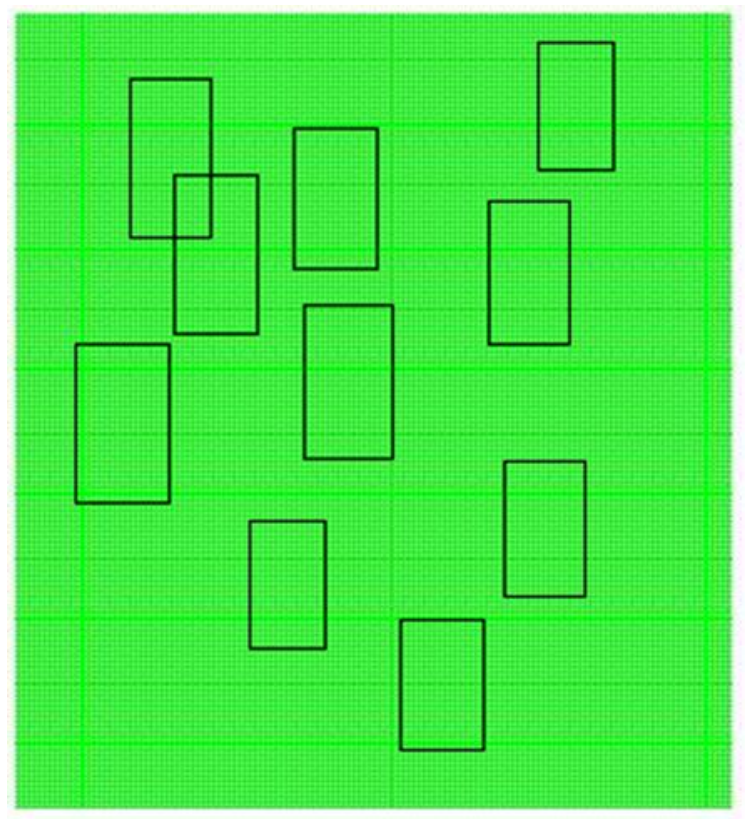

Figure 5.5 Schematic diagram for each specimen

\subsubsection{Results discussion}

The plots listed in Figure 5.6 and Figure 5.7 show the different failure pattern in 200 psi and 500 psi confining pressures respectively. Although all the conditions are kept the same, the failure patterns still propagate in many different ways in both Figure 5.6 and Figure 5.7. These phenomena also verified that the spatial variance exists in reality. There were different relatively weak zones present in different locations. 


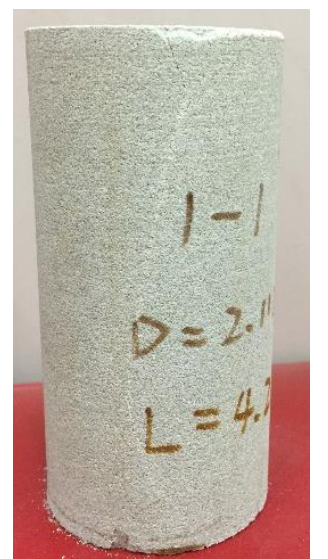

1-1-200

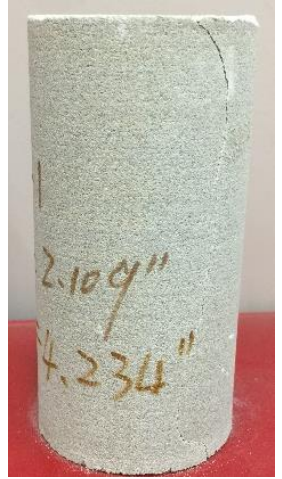

6-1-200

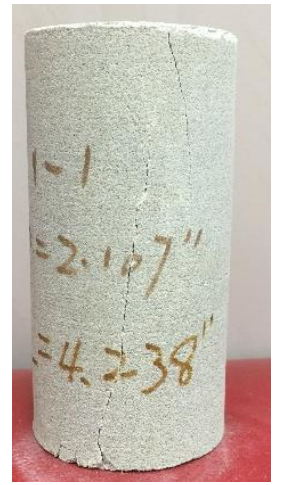

11-1-200

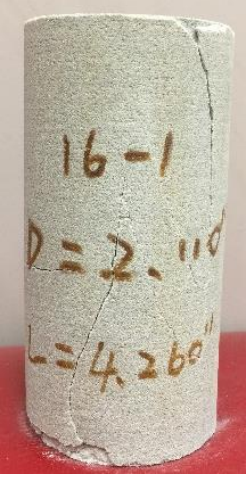

16-1-200

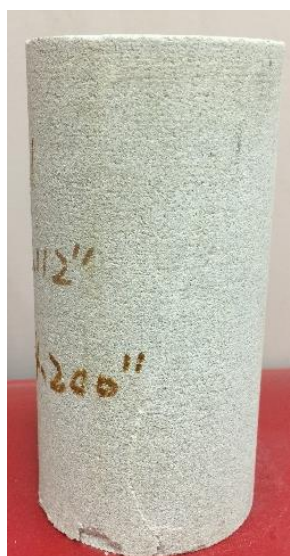

2-1-200

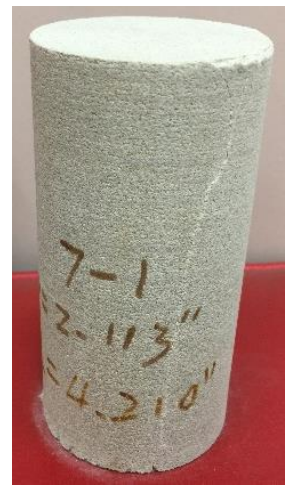

7-1-200

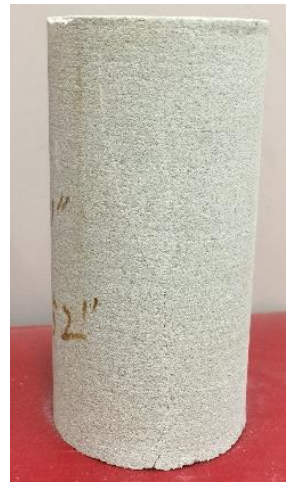

12-1-200

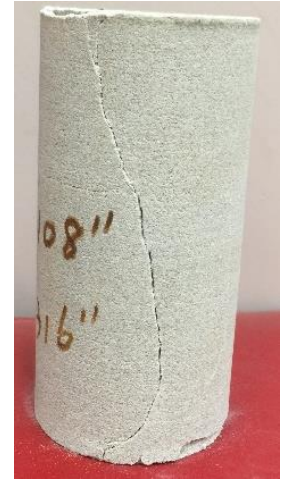

17-1-200

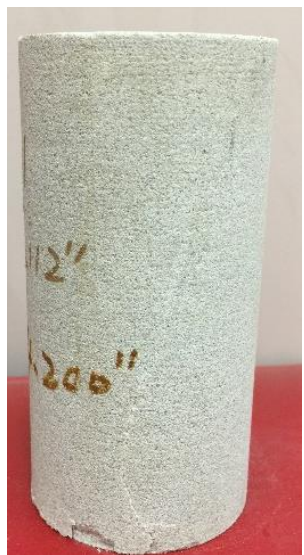

3-1-200

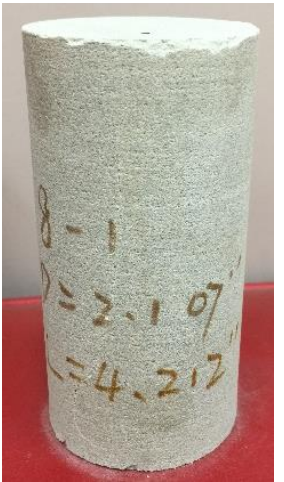

8-1-200

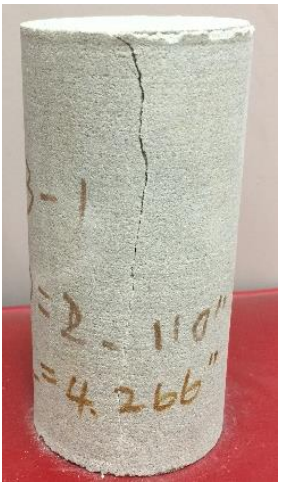

13-1-200

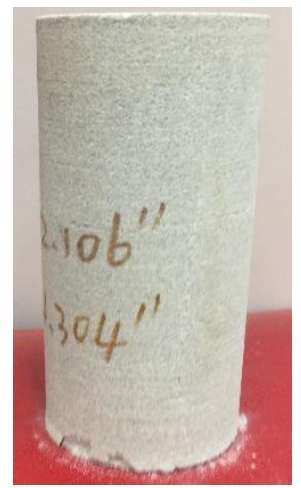

18-1-200

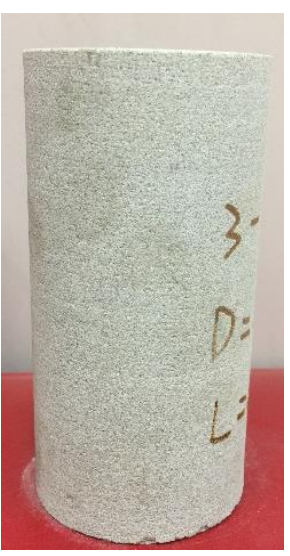

4-1-200

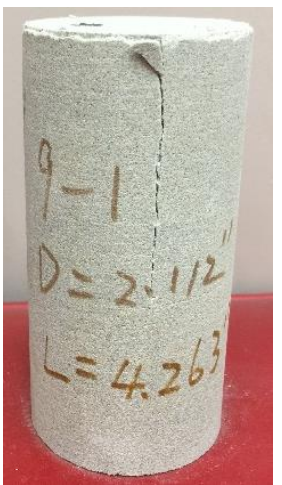

9-1-200

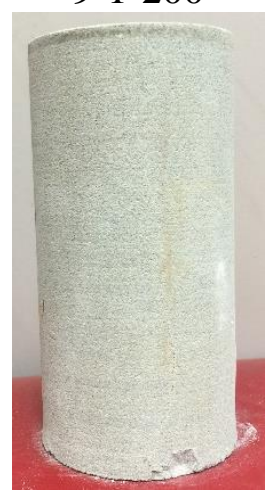

14-1-200

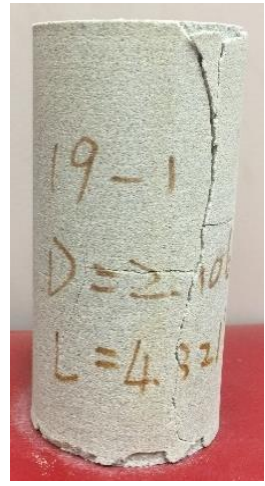

19-1-200

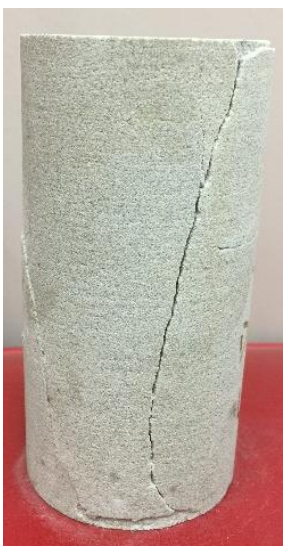

5-1-200

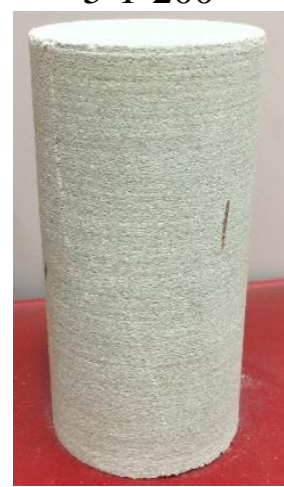

10-1-200

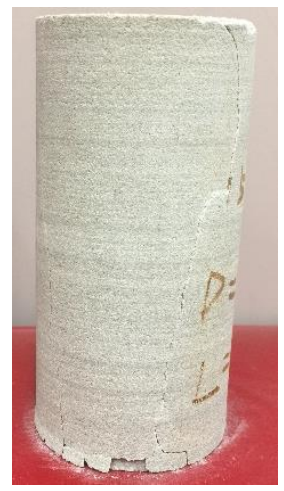

15-1-200

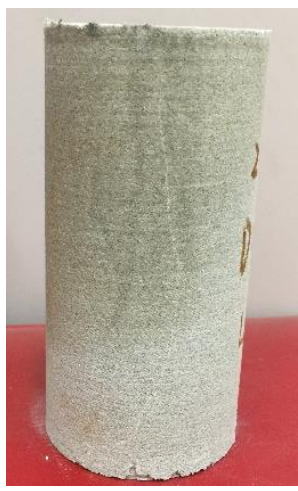

20-1-200 


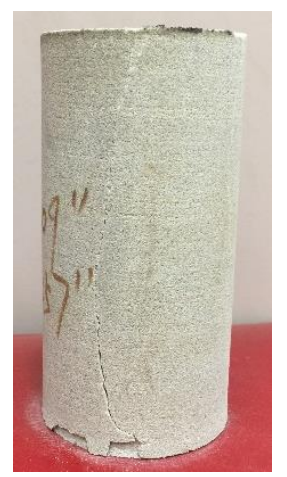

21-1-200

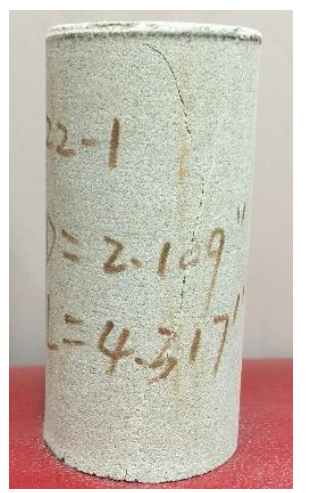

22-1-200

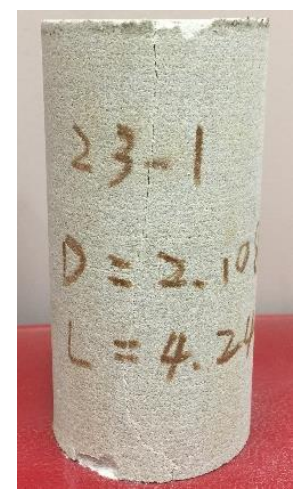

23-1-200

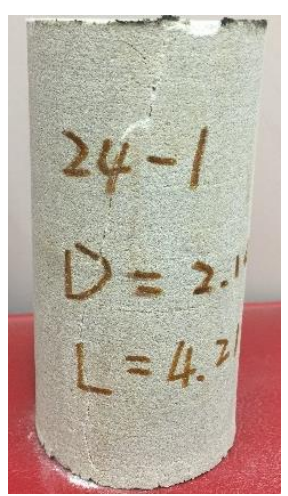

24-1-200

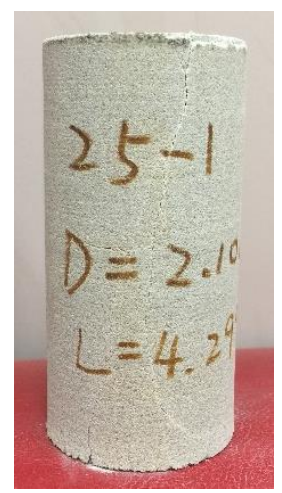

25-1-200

Figure 5.6 Failure pattern for each specimen under 200psi confining pressure

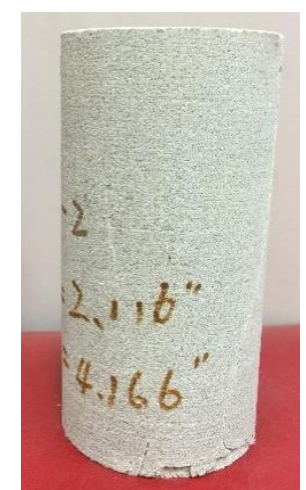

$1-2-500$

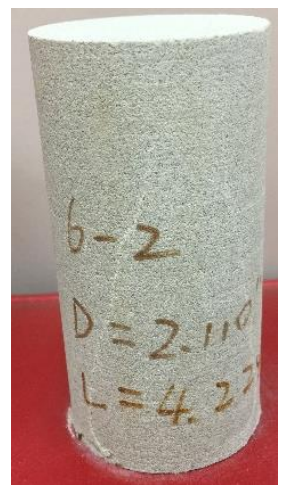

6-2-500

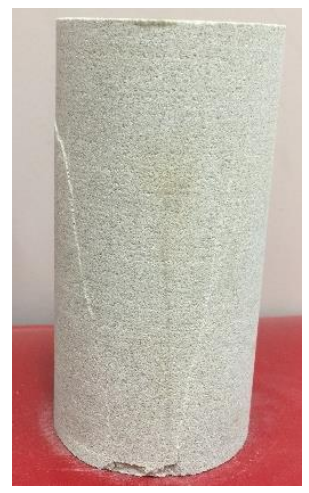

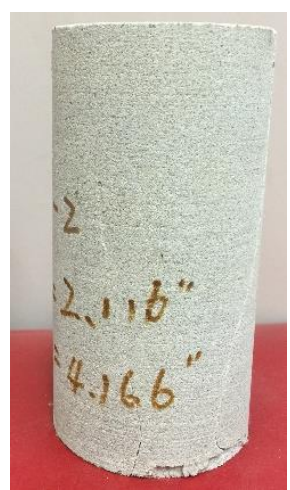

2-2-500

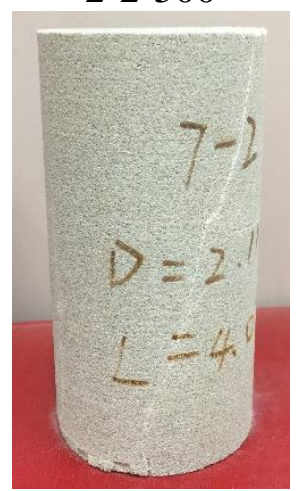

7-2-500

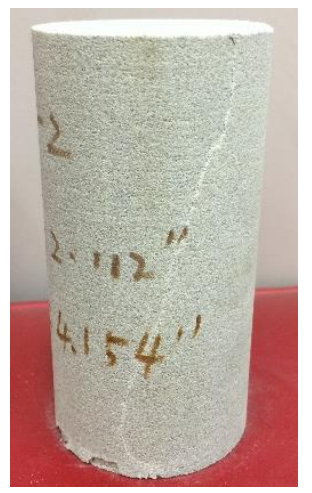

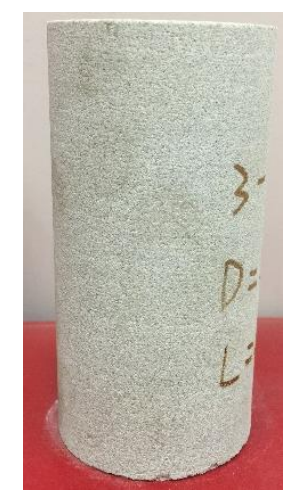

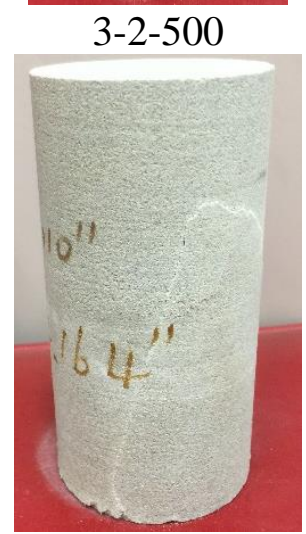

8-2-500

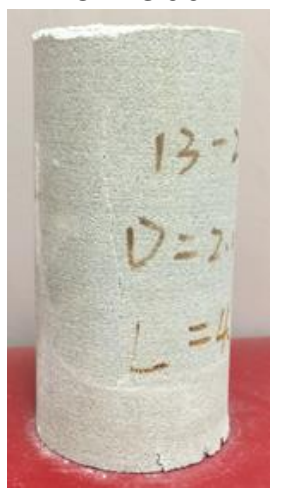

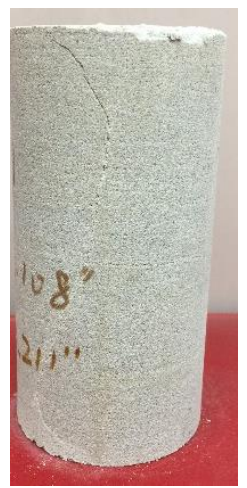

4-2-500

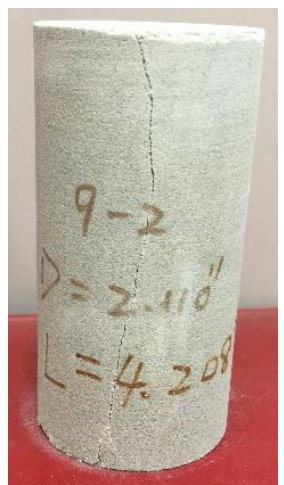

9-2-500

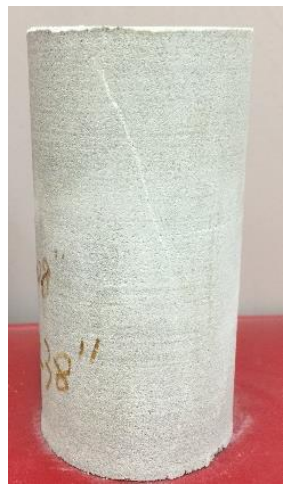

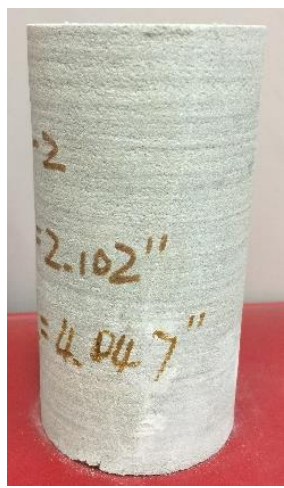

5-2-500

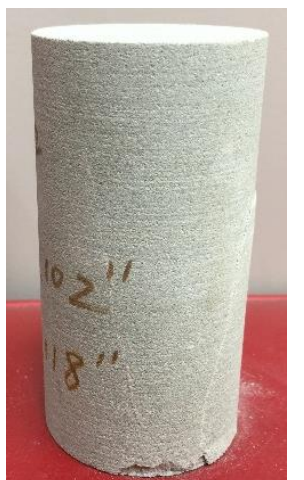

$10-2-500$

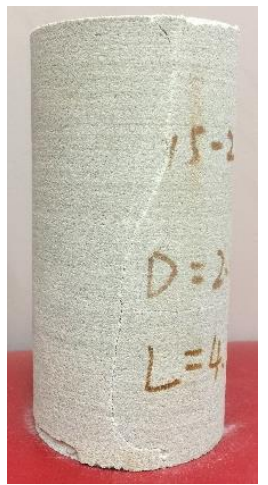



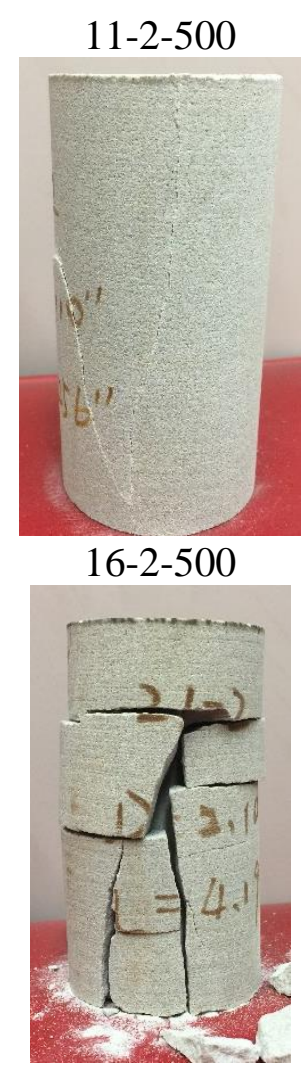

$21-2-500$

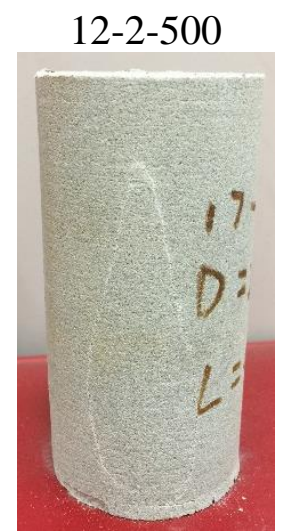

$17-2-500$

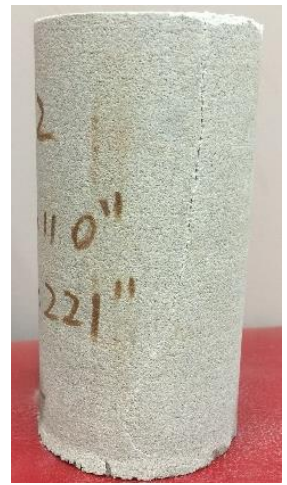

$22-2-500$

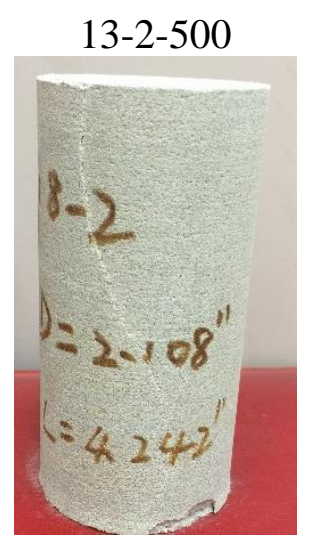

$18-2-500$

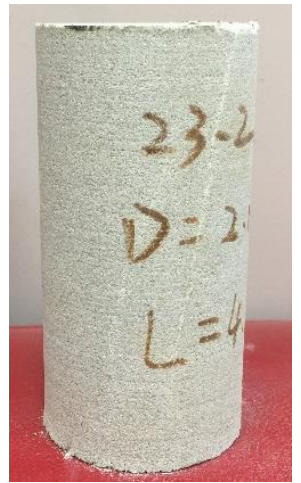

$23-2-500$

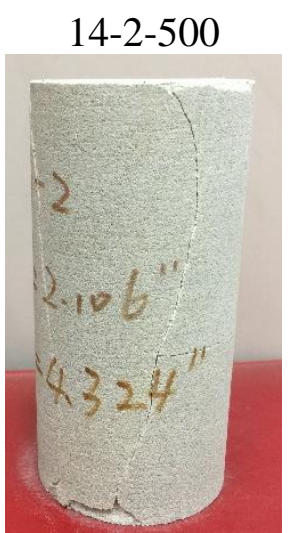

$19-2-500$

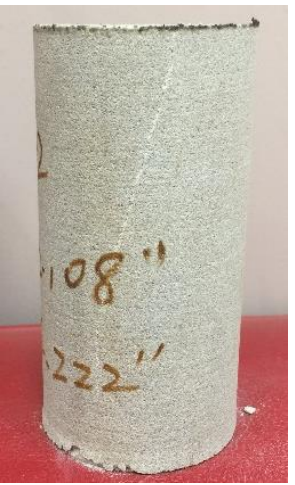

$24-2-500$
15-2-500

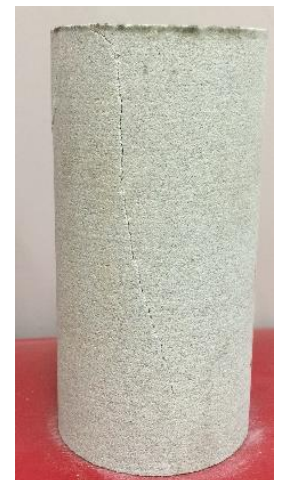

$20-2-500$

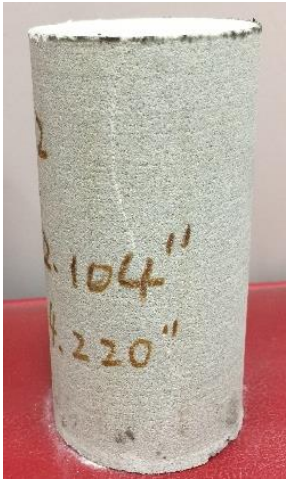

$25-2-500$

Figure 5.7 Failure pattern for each specimen under 200psi confining pressure

Figure 5.8 and 
Table 5.4 show the strength comparison between laboratory test and numerical simulation for the 50 sandstone specimens drilled from the large rock. For both the specimens under 200 psi and 500 psi confining pressure, Slight difference showed up between laboratory tests and numerical simulation for strength. From the linear regression analysis (the trendline is $y \approx x$ ), the coefficient of determination for these two groups are 0.9962 and 0.9899 respectively, which means the results obtained from the laboratory tests agree with the numerical models perfectly.

From the summary in 
Table 5.4, the mean values for the laboratory tests and numerical simulations under $200 \mathrm{psi}$ confining pressure are 13,522.79 psi and 13,908.68 psi respectively; the mean values for the laboratory tests and numerical simulations under 500 psi confining pressure are 16,181.06 psi and 16,162.08 psi respectively. The maximum error between these two models is only around $3 \%$.
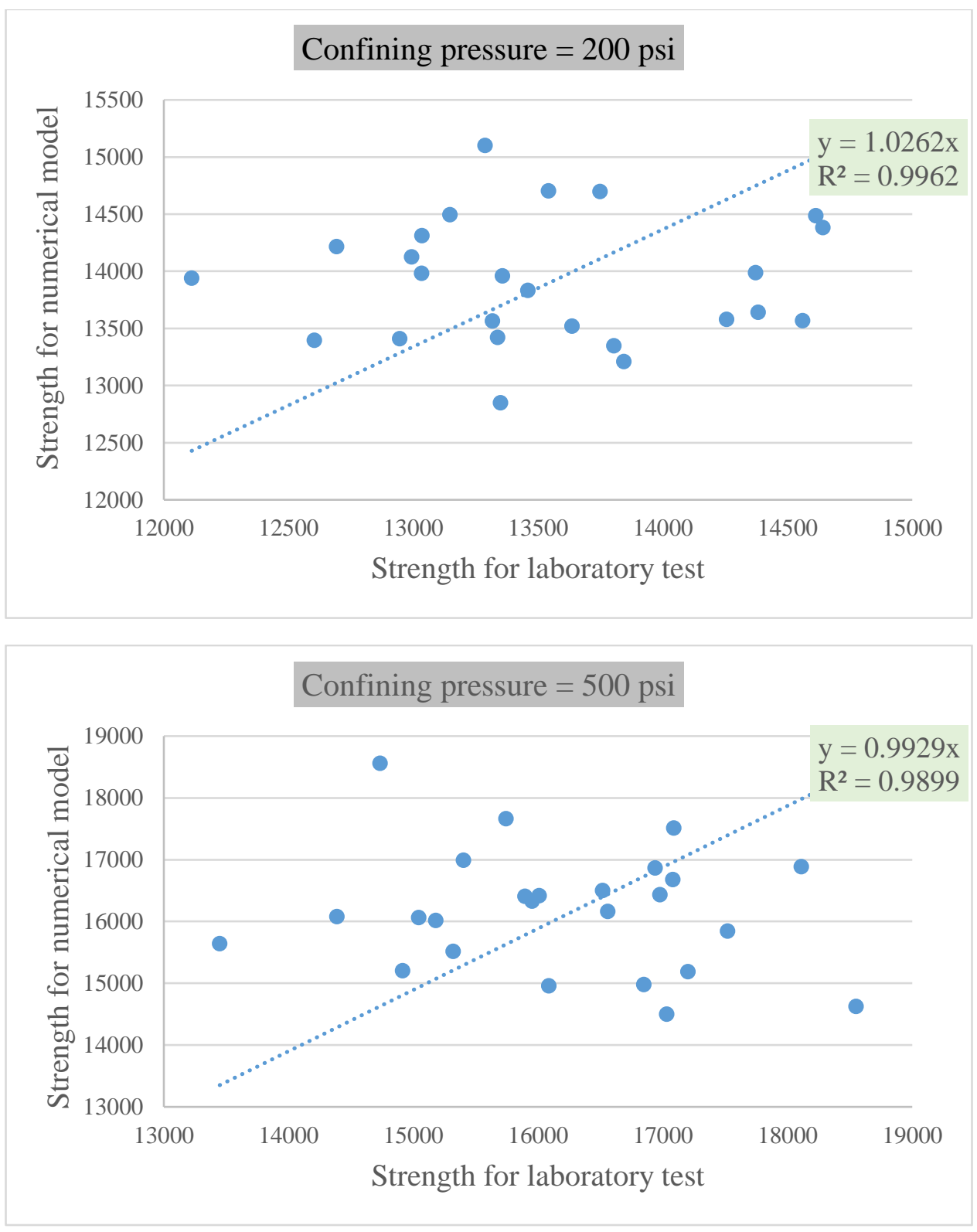

Figure 5.8 Strength comparison between laboratory test and numerical simulation (Unit: psi) 
Table 5.4 Strength comparison between laboratory test and numerical simulation

\begin{tabular}{|c|c|c|c|c|}
\hline \multirow{2}{*}{$\begin{array}{c}\text { Confining } \\
\text { pressure } \\
(\mathbf{p s i})\end{array}$} & \multicolumn{2}{|c|}{ Mean value (psi) } & \multicolumn{2}{c|}{ Standard deviation } \\
\cline { 2 - 5 } & $\begin{array}{c}\text { Laboratory } \\
\text { test }\end{array}$ & $\begin{array}{c}\text { Numerical } \\
\text { simulation }\end{array}$ & $\begin{array}{c}\text { Laboratory } \\
\text { test }\end{array}$ & $\begin{array}{c}\text { Numerical } \\
\text { simulation }\end{array}$ \\
\hline 200 & $13,522.79$ & $13,908.68$ & 668.11 & 545.60 \\
\hline 500 & $16,181.06$ & $16,162.08$ & $1,204.64$ & 974.94 \\
\hline
\end{tabular}

For the standard deviation in 
Table 5.4, the results obtained from the numerical simulations are much smaller than the results obtained from the laboratory test. The reason for this is that, for the mathematical model, no discontinuities in the rock block were assumed. The spatial variance's existence is only due to the material property. However, many other factors can influence the strength in a real case. Therefore, the variance is much higher in laboratory than in the ideal numerical models.

\subsection{Conclusions}

The main goal of this chapter was to study the strength difference between laboratory test and numerical simulation. Conclusions are summarized as follows:

The different failure patterns obtained from laboratory tests showed that different weaklinks exist in different locations, which verified Weibull's weakest-link conclusion and verified the inherent existence of spatial variance.

The comparison results between the laboratory tests and numerical simulations match each other in a good way; the maximum error of the mean values for the laboratory tests and numerical simulations is only around 3\%, which is acceptable per this research's expectation.

Due to the assumption that there are no discontinuities existing in the rock block, the spatial variance's existence is only due to the material property; the difference of standard deviations for laboratory tests and numerical simulations are strongly present. However, the standard deviation value of numerical simulations is lower than the values of laboratory tests, which is reasonable given that the other factors were ignored that may cause the strength to be unstable. 


\section{CHAPTER 6 \\ CASE STUDY: PROBABILISTIC EXAMINATION OF SPATIAL VARIANCE EFFECTS ON THREE DIFFERENT FIELD SCALED MODELS}

\subsection{Introduction}

Rock that is heterogeneous in formation significantly influences the failure of rock. Both macroscopic and microscopic heterogeneity influences the physical-mechanical behavior. At the microscopic level, the grain size and shape distribution cause the specimen to show non-linear behavior. At the macroscopic level, heterogeneity affects the strength of the rock. For example, a coal mine entry using a single material property will produce inaccurate safety factors (Kim \& Gao, 1995; Tang, 1997). Similarly, rockmass strength is inherently weaker than intact rock, which is due to the heterogeneous distribution of rock.

In rock structures, the weak formation interacts on a different scale and is extremely complex in its formation. However, research efforts have focused on stress field investigation and failure criterions of materials. For example, the strength of pillars is critical for mining operations in underground coal and hard rock mines. However, a unit value is often only considered when performing a stability calculation. A significant research effort within these past studies has focused on pillar stability and design using theoretical formulation (Bieniawski, 1968), numerical simulation (Recio-Gordo \& Jimenez, 2012), and empirical method (Soltani, 2015). The problem is that these studies always use deterministic strength values to represent the coal strength of the whole pillars, which ignores the inherent variability in rockmass properties. In order to analyze rockmass behavior, the inherent variability of rockmass mechanical properties, 
such as the strength and deformation properties, must be assessed probabilistically for the whole area.

Several researchers such as Weibull used weakest-link theory to explain the inherent variability of a material (Weibull, 1939). Salamon, Peng, and Scovazzo used finite element method, including variable material properties, on applications such as pillar design in underground mines (Peng, 1992; Salamon \& Munro, 1967; Scovazzo, 1992). Priest, Hudson, etc. discovered that the distributions of joints in rockmass follow exponential distributions (Einstein, 1983; La Pointe, 1985; Priest \& Hudson, 1976). Yegulalp and Kim showed that the mechanical properties of intact rock matches the Type III Extreme Value distribution of the smallest value (Yegulalp \& Kim, 1994). The grain sized rock material properties are typically assigned with random models; however, the process of formation of rockmass necessitates the random distributions of each physical indicator to be spatially correlated. Few studies have considered the spatial correlation factor combined with random rock material properties.

In the methodology introduced previously, Chapter 4 proposed one probabilistic approach by using Extreme Value distribution and spatial correlation to assess the variability of rockmass mechanical properties. This method treats rockmass properties as spatially correlated random variables. The study in this chapter focuses mainly on the following three field scaled models:

(1) One two-dimensional finite difference longwall mine model, which was analyzed by using the random method. The main goal of this field scaled model is to verify the necessity of using the stochastic method in longwall mining system.

(2) Another three-dimensional longwall mine model, which was analyzed by using the proposed approach mentioned above. There is an expected influence of random field data with spatial correlation factor on entry roofs in the longwall mine system. 
(3) One pillar scaled model, which was created in this chapter to only consider the immediate roof and coal seam parts in completely random conditions. This is to extract several laboratory-scaled specimens from the pillar, which is in preparation for future stochastic analysis for laboratory scaled models.

\subsection{Case one: Longwall panel model}

\subsubsection{Model description}

In the previous sections, the application of statistics mainly focused on the sample scaled rocks. The previous studies already concluded that the random model has an advantage in estimating the failure types of rocks, especially considering the correlation factor. In this section, extending this statistical theory into one three-dimensional longwall panel model was considered.

Due to the symmetric characteristic and the limitation of the computer calculation, the study here only created one-quarter of the longwall panel with three layers. This included a sandstone layer on the top, siltstone layer on the bottom, and one coal seam in the middle. As seen in Figure 6.1, the room and pillar geometries are not very complex. The series of pillars are in a regular rectangular pattern. The details of the geometry and dimensions are as follows:

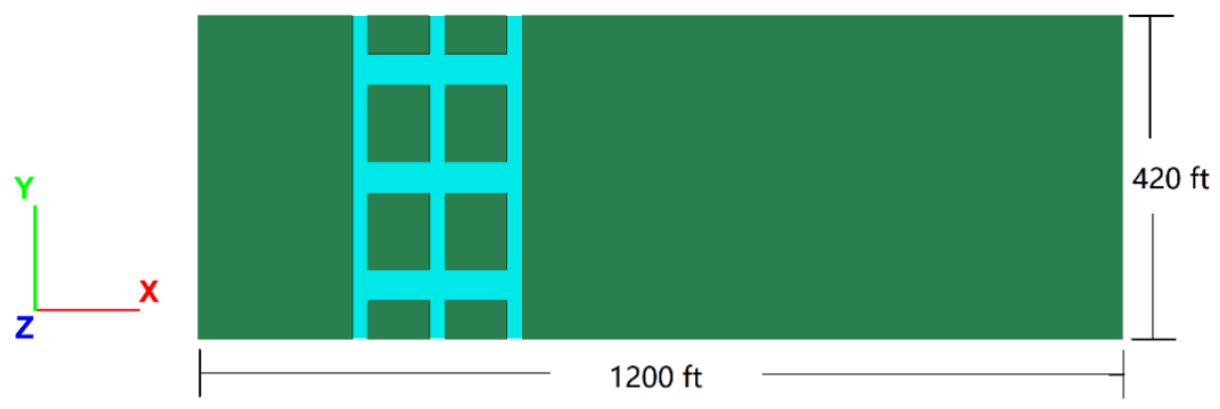

Figure 6.1 Geometry for the longwall panel model

Note:

$$
\begin{array}{ll}
\text { Panel }_{\text {Lwidth }}=80 \mathrm{ft} . & \text { Pillar }_{\text {Llength }}=80 \mathrm{ft} . \\
\text { Panel }_{\text {Rwidth }}=420 \mathrm{ft} . & \text { Panel }_{\text {Rlength }}=780 \mathrm{ft} .
\end{array}
$$




$$
\begin{aligned}
\text { Entry Width }=20 \mathrm{ft} . & \text { Crosscut }=40 \mathrm{ft} . \\
\text { Height }_{\text {main roof }}=50 \mathrm{ft} . & \text { Height }_{\text {immediate roof }}=50 \mathrm{ft} . \\
\text { Height }_{\text {coal seam }}=9 \mathrm{ft} . & \text { Height }_{\text {floor }}=100 \mathrm{ft} .
\end{aligned}
$$

Table 6.1 Strata material properties

\begin{tabular}{|c|c|c|c|c|c|c|c|c|}
\hline $\begin{array}{c}\text { Rock } \\
\text { strata }\end{array}$ & $\begin{array}{c}\mathbf{H} \\
(\mathbf{i n c h})\end{array}$ & $\begin{array}{c}\mathbf{\rho} \\
\left(\mathbf{s n a i l s}_{\mathbf{i n n}} \mathbf{3}^{2}\right.\end{array}$ & $\mathbf{v}$ & $\begin{array}{c}\mathbf{K} \\
\left(\times 10^{6} \mathbf{p s i}\right)\end{array}$ & $\begin{array}{c}\mathbf{G} \\
\left(\times 10^{4} \mathbf{p s i}\right)\end{array}$ & $\begin{array}{c}\mathbf{c} \\
(\mathbf{p s i})\end{array}$ & $\begin{array}{c}\boldsymbol{\varphi} \\
\left({ }^{\circ}\right)\end{array}$ & $\begin{array}{c}\boldsymbol{\sigma}_{\mathbf{t}} \\
(\mathbf{p s i})\end{array}$ \\
\hline $\begin{array}{c}\text { Main } \\
\text { Roof }\end{array}$ & 600 & 0.0002337 & 0.15 & 1.43 & 130 & 1562 & 35 & 130 \\
\hline $\begin{array}{c}\text { Immediate } \\
\text { Roof }\end{array}$ & 600 & 0.0002337 & 0.15 & 0.95 & 87 & 1562 & 35 & 130 \\
\hline Seam & 108 & $1.31 \mathrm{e}-4$ & 0.25 & 0.33 & 20 & 433 & 30 & \\
\hline Floor & 1200 & 0.0002337 & 0.15 & 1.19 & 1.09 & 2681 & 35 & 200 \\
\hline
\end{tabular}

Note: $H$ is the height of each layers, inch;

$\rho$ is density, snails/in3;

$v$ is Poisson's ratio;

$G$ is Shear modulus, psi;

$K$ is Bulk modulus, psi;

$\sigma_{t}$ is Tensile strength, psi;

$c$ is cohesion, psi, and;

$\varphi$ is friction angle, degree.

Figure 6.2 shows the roller boundary conditions assigned at the side and bottom of the model.

The three loads applied to this longwall panel model are as follows:

a) Gravitational acceleration ( $\left.386 \mathrm{in} / \mathrm{sec}^{2}\right)$ applied to all the elements;

b) The overburden load applied to the top surface, which has been equivalent to a static pressure $\sigma_{v 0}(200 \mathrm{psi})$;

c) The vertical stress $\sigma_{v}$ and horizontal stress $\sigma_{h}$ applied to each layer, with the relationship between them is described as:

$$
\sigma_{h}=\frac{v}{1-v} \sigma_{v}
$$
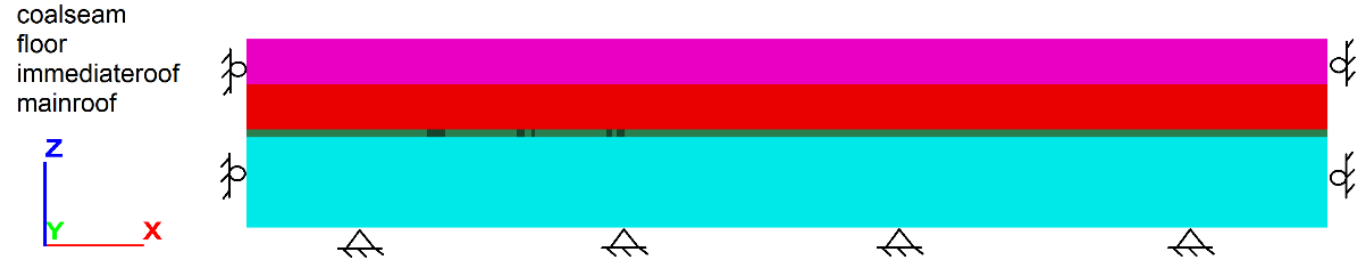

Figure 6.2 Boundary condition for the longwall panel model 


\subsubsection{Result discussions}

After applying the development load onto the longwall panel, most of the high stress concentration occurred near the entries and crosscuts. In order to prove the feasibility of this model, a comparison of the vertical stresses between FLAC3D (Figure 6.3a) and LAMODEL (Figure 6.3b) was considered.

The maximum stress for the adjacent area of pillars is around 25 psi in FLAC3D and 26 psi in LAMODEL. The values are slightly different, but otherwise quite close to each other. Therefore, the model is acceptable for performing the future stochastic analysis.

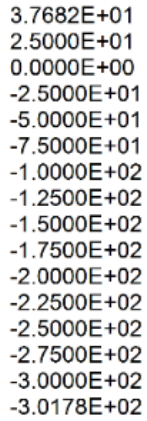

$-1.0000 \mathrm{E}+02$

$-1.2500 \mathrm{E}+02$

$-1.5000 \mathrm{E}+02$

$-1.7500 \mathrm{E}+02$

$-2.0000 \mathrm{E}+02$

$-2.2500 \mathrm{E}+02$

$-2.5000 \mathrm{E}+02$

$-2.7500 \mathrm{E}+02$

$-3.0000 \mathrm{E}+02$

$-3.0178 \mathrm{E}+02$

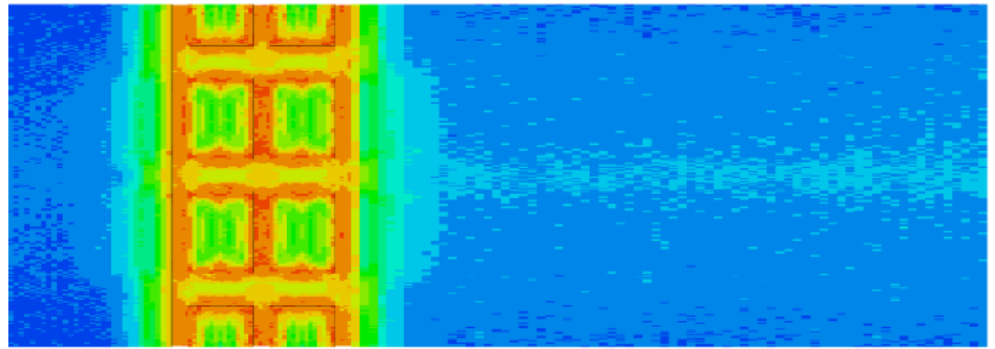

(a) zz-stress for the coal seam area in FLAC3D (Unit: psi)

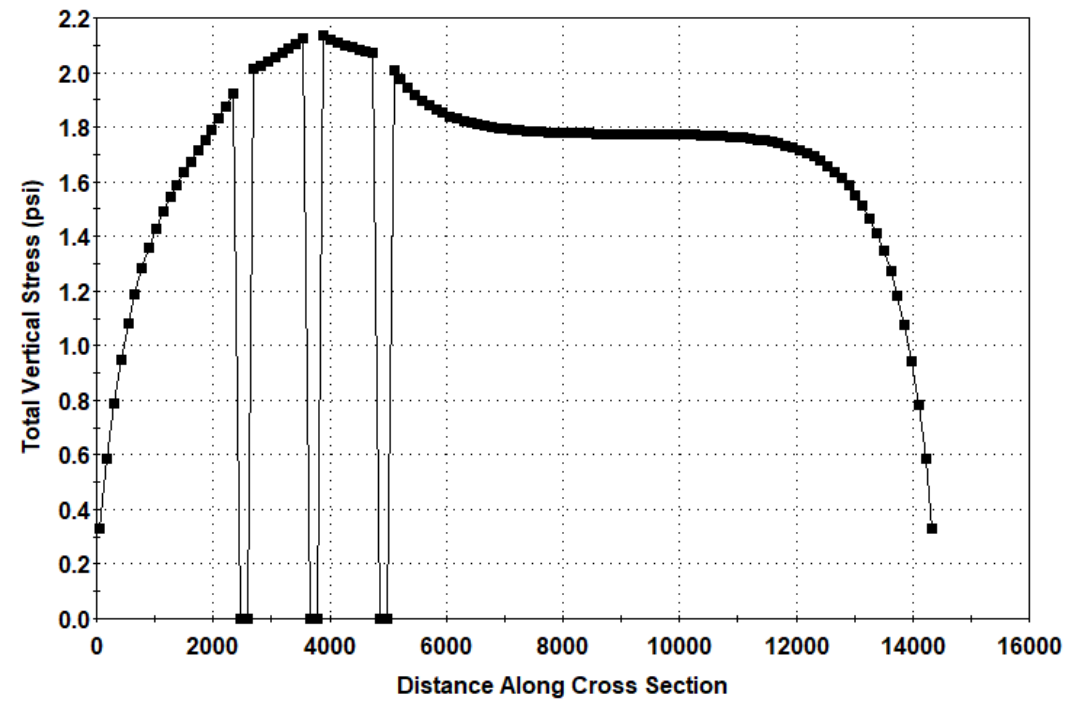

(b) zZ-stress for the coal seam area in FLAC3D (Unit: psi)

Figure 6.3 Comparison of vertical stress between FLAC3D and LAMODEL 
Figure 6.4 shows the simulation of the comparison between the deterministic and stochastic models. From the literature review, we discovered that most fatal accidents occur in the immediate roof and coal seam. As such, they only set the immediate roof and coal seam layers into normal distribution, shown in Figure 6.4(b). In order to track the vertical stress in different steps, this study set eleven grid points along the Y axis for four pillars ordered into A B C D, shown in Figure 6.4(c).

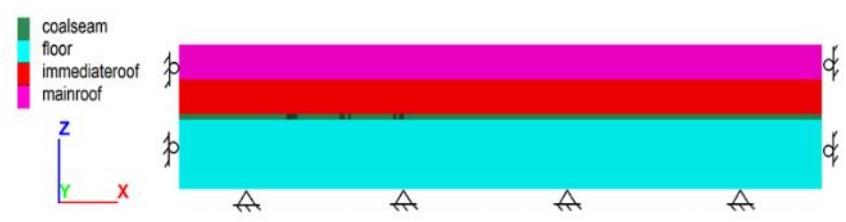

(a) Laminations for longwall model

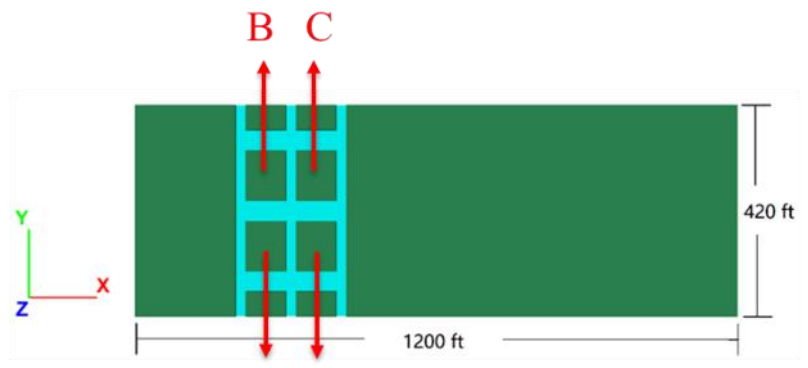

A D

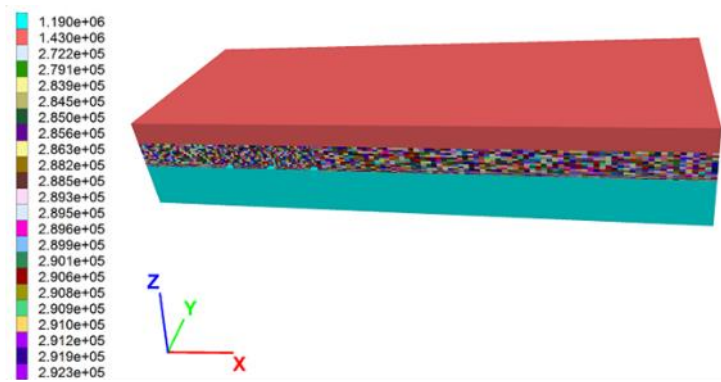

(b) Material property distribution example for stochastic model (Unit: psi)

Panel $_{\text {width }}=80 \mathrm{ft}$

Panel $_{\text {width }}=420 \mathrm{ft}$

Entry Width $=20 \mathrm{ft}$

Height $_{\text {main roof }}=50 \mathrm{ft}$

Height $_{\text {coal seam }}=9 \mathrm{ft}$

$$
\begin{gathered}
\text { Pillar }_{\text {length }}=80 \mathrm{ft} \\
\text { Panel }_{\text {length }}=780 \mathrm{ft} \\
\text { Crosscut }=40 \mathrm{ft} \\
\text { Height }_{\text {immediate roof }}=50 \mathrm{ft} \\
\text { Height }_{\text {floor }}=100
\end{gathered}
$$

(c) Crosscut section for coal seam layer

Figure 6.4 Schematic diagram for quarter part of one longwall panel

In Figure 6.5, the orange dots show the vertical stresses tracking from the different grid points. Solid lines represent the pillar with uniform property; the dashed lines represent the pillars with random values, which have normal distributions. After fitting the curve, we noted that all the curves were identical. The right corner plot in Figure 6.5 is the enlarged view obtained from JMP software, a suite of computer programs for statistical analysis developed by SAS Institute. The green boxes are the mean value and standard deviation for each location. 
Although the mean value curve fitting is almost the same for each situation, the differences between the models with deterministic and random values for each pillar and each location are randomly distributed; these behave like "sin" or "cos" waves, but not exactly with the same shapes. This field scaled model only considered the random material properties without the spatial correlation factor.

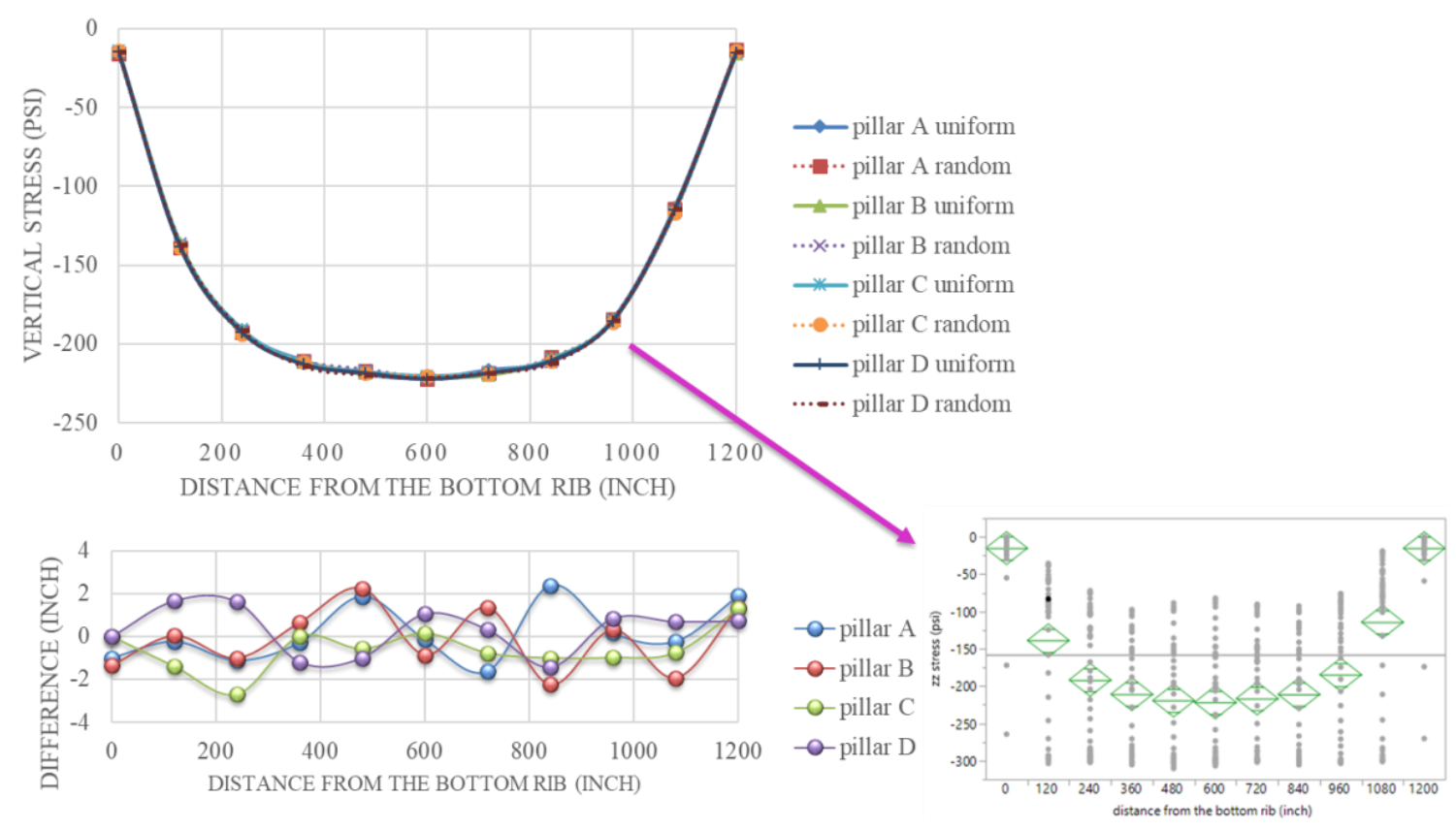

Figure 6.5 Difference of stress distribution along each pillar between deterministic and stochastic model

\subsection{Case two: Longwall mine model study}

\subsubsection{Model description}

The mine extracts $7 \mathrm{ft}$. thick Pittsburgh coal seam by longwall mining method. The depth of cover in the area is $833 \mathrm{ft}$. The measured in-situ maximum and minimum horizontal stresses are 3,080.6 psi and $2179.92 \mathrm{psi}$, respectively. The in-situ maximum horizontal stress is oriented at $30^{\circ}$ to the longitudinal axis of the longwall panel. As shown from the representative lithology 
shown in Figure 6.6, the thickness of the immediate roof is $3 \mathrm{ft}$. The thickness of the floor is 260 $\mathrm{ft}$, which is a combination of shale, claystone and sandy shale.

The numerical model was developed using a finite difference software, FLAC3D. As shown in Figure 6.6, the model boundaries were placed at the center of the panel along its width and at an enough distance along the panel length to have negligible impact on the results obtained in the area of interest. The final dimensions were $3,559 \mathrm{ft} \times 820 \mathrm{ft} \times 498 \mathrm{ft}$. A total of 1,596,540 zones was generated in this simulation. As limited computing resources are available, only part of the overburden has been modelled in the mesh. The pressure, which equals the gravity of the remaining covers, is applied on the top layer.
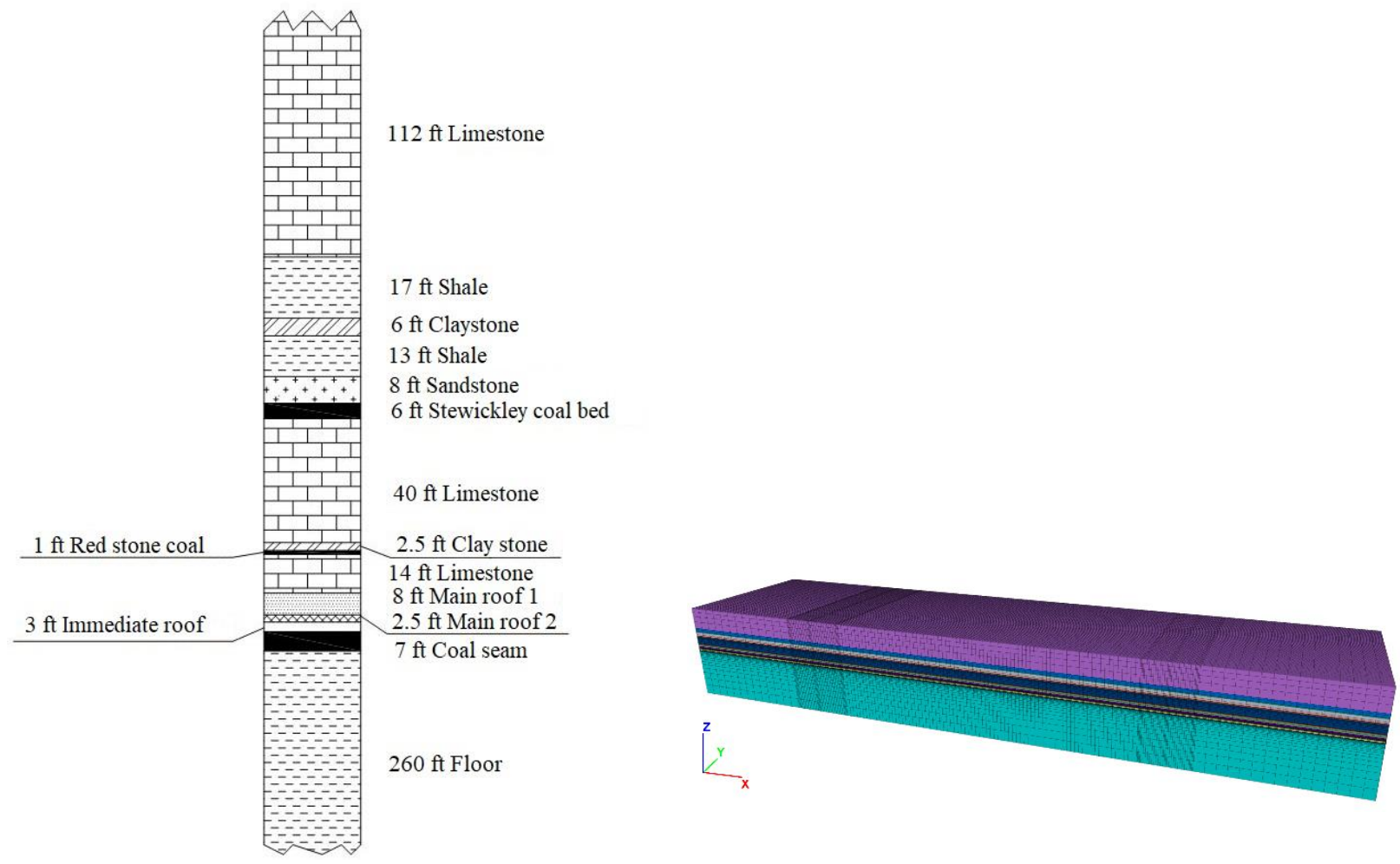

Figure 6.6 Representative lithology used in the FLAC3D model

Table 6.2 lists the laboratory material properties of rocks. The strength of intact rock obtained from experimental data is conservative to estimate the strength of rockmass directly. 
Therefore, consideration of the strength reduction factor from intact rock to real rockmass is necessary (Hoek, 1983). Here, this study estimates the rockmass compressive and tensile strengths by using the corresponding laboratory values listed in Table 6.2 multiplied by the reduction factor.

To study the influence of the spatial variance on the field scaled model, this research considers two different approaches for this model:

(1) Traditionally deterministic method, and

(2) Extreme Value stochastic method, including the spatial correlation factor.

Table 6.2 Laboratory material properties

\begin{tabular}{|c|c|c|c|c|}
\hline Rock Type & $\begin{array}{c}E \\
\left(\times 10^{5} \mathrm{psi}\right)\end{array}$ & $v$ & $\begin{array}{l}\text { UCS } \\
\text { (psi) }\end{array}$ & $\begin{array}{c}\tau \\
(\mathbf{p s i})\end{array}$ \\
\hline Limestone & 74.26 & 0.26 & $22,131.35$ & $1,759.31$ \\
\hline Claystone & 13.83 & 0.3 & $4,709.38$ & 398.85 \\
\hline Sandy shale & 24.19 & 0.3 & $7,275.11$ & $1,096.49$ \\
\hline $\begin{array}{c}\text { Shale } \\
\text { (lower roof) }\end{array}$ & 14.25 & 0.3 & $4,372.90$ & 52.21 \\
\hline $\begin{array}{c}\text { Shale } \\
\text { (upper roof) }\end{array}$ & 13.34 & 0.3 & $7,159.08$ & 704.88 \\
\hline Rider coal & 5.80 & 0.39 & $2,104.50$ & 292.98 \\
\hline Coal seam & 6.82 & 0.39 & $4,313.43$ & 321.98 \\
\hline $\begin{array}{l}\text { Shale or Claystone } \\
\text { (in the floor) }\end{array}$ & 13.78 & 0.3 & $5,949.46$ & 335.04 \\
\hline \multicolumn{5}{|l|}{ Note: } \\
\hline
\end{tabular}

Table 6.3 gives all the unique material property values used in the model for both approaches. For the model with spatial correlated random parameters, cohesion and internal friction are the primary factors that affect failure propagation and are randomly assigned to the model. Because 
of the difficulties of performing data collection in the real site, we assumed the standard deviation $\sigma$ and correlation parameters $a$ and $b$ were assumed here; however, the mean value $\mu$ applied on the model is still based on the laboratory test.

Table 6.3 Random values for the model with spatial variance

\begin{tabular}{|c|c|c|c|c|c|c|c|c|}
\hline \multirow{2}{*}{ Rock Type } & \multicolumn{4}{|c|}{ Cohesion (psi) } & \multicolumn{4}{c|}{$\begin{array}{c}\text { Internal friction angle } \\
\text { (degree) }\end{array}$} \\
\cline { 2 - 10 } & $\boldsymbol{\mu}_{\boldsymbol{c}}$ & $\boldsymbol{\sigma}_{\boldsymbol{c}}$ & $\boldsymbol{a}_{\boldsymbol{c}}$ & $\boldsymbol{b}_{\boldsymbol{c}}$ & $\boldsymbol{\mu}_{\boldsymbol{f}}$ & $\boldsymbol{\sigma}_{\boldsymbol{f}}$ & $\boldsymbol{a}_{\boldsymbol{f}}$ & $\boldsymbol{b}_{\boldsymbol{f}}$ \\
\hline Immediate roof & 1,562 & 15.6 & 2 & 4 & 35 & 0.35 & 2 & 4 \\
\hline Coal seam & 433 & 4.3 & 2 & 4 & 30 & 0.3 & 2 & 4 \\
\hline
\end{tabular}

This research created the realistic random field database based on the Extreme Value stochastic model, adding two scaler-measured parameters from both horizontal and vertical directions to control the spatial correlation length (Equation 4.3). This model considers a few cutting sequences to investigate the influence of spatial variance over the roof behavior. The total excavation depth used here is $50 \mathrm{ft}$.

Due to computational resource limitations, this study only set the immediate roof layer in the spatially correlated random condition. Figure 6.7 shows a realization of a random field for both cohesion and friction.

In the correlation parameters listed in Table 6.3, for both the cohesion and friction distributions, high values mix randomly with lower values, and the values in adjacent areas process more correlated material properties than the values in distant areas. The spatial correlation parameters for cohesion and friction are kept constant; therefore, the correlation characteristic of these two parameters is kept the same. The angle of the principal axis is set to zero degrees, which is a line associated with the correlated circle or ellipse. Therefore, the direction of the correlation area for this model is horizontal. 


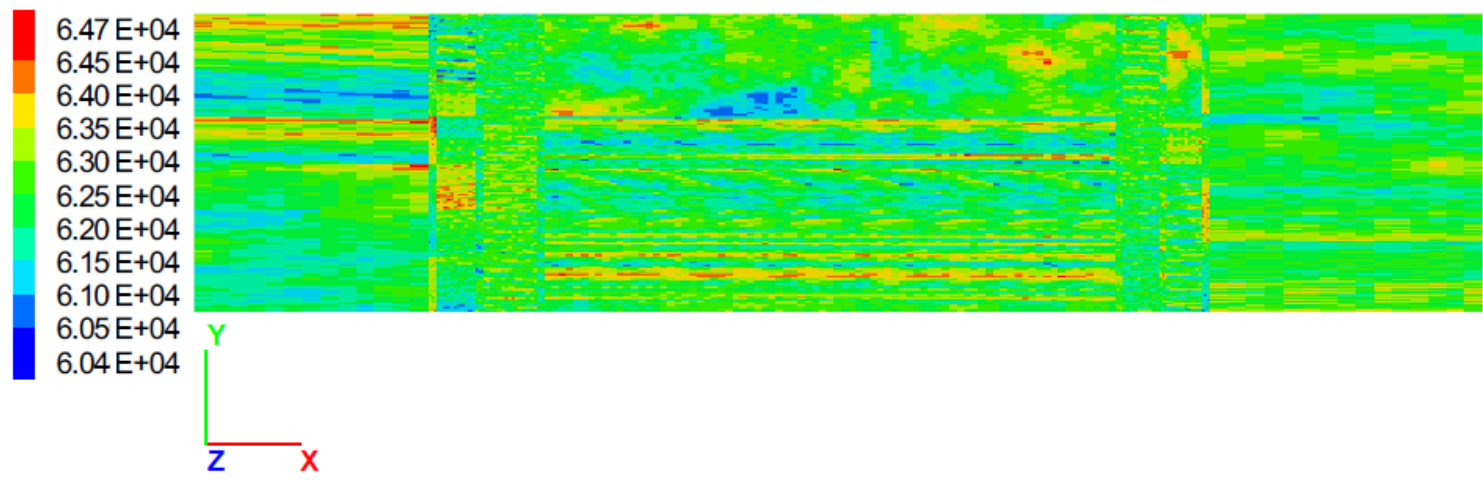

Random field for the cohesion parameter (Unit: psf)

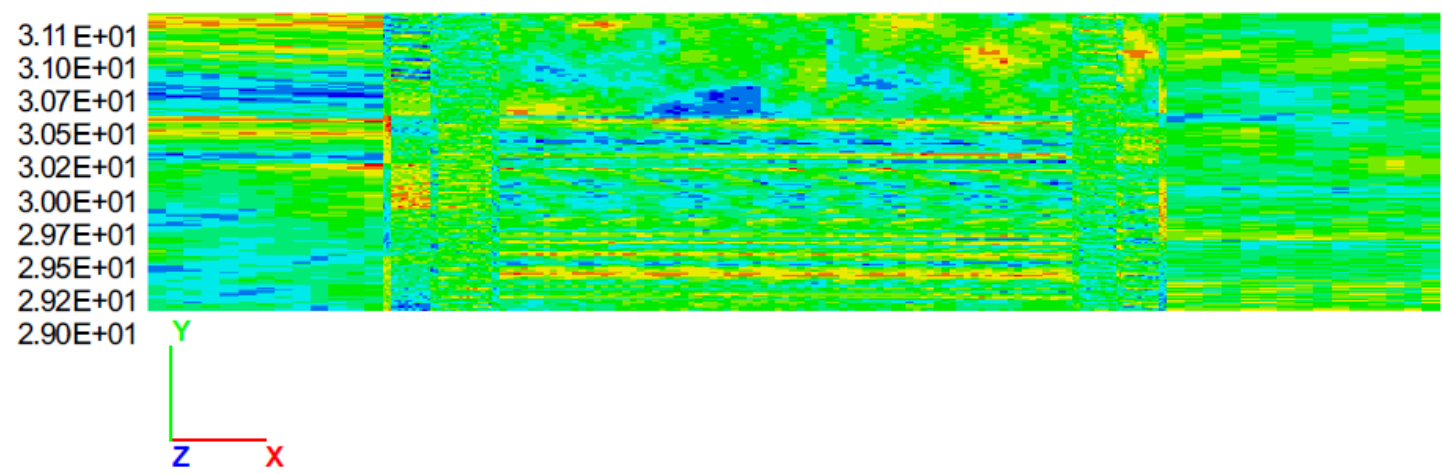

Random field for the friction angle parameter (Unit: degree)

Figure 6.7 Random field's distributions for the two parameters

\subsubsection{Result discussions}

In order to simulate the extraction from the actual field scaled model, this research established the in-situ stress field in the model as shown in Figure 6.8. The maximum stress for this model was $1,235.72$ psi. The global height of this model was $1160 \mathrm{ft}$; as such, the overburden stress for the whole model is $1.1 \mathrm{psi} / \mathrm{ft} \times 1,160 \mathrm{ft} \times 144=183,744 \mathrm{psf}$. For verification purposes, the in-situ stresses obtained from numerical simulation matched with the values obtained from theoretical estimation. The minor difference is due to the approximate solution of numerical analysis. Therefore, it is reasonable to use these input parameters to assess the mechanical behavior of this model. 


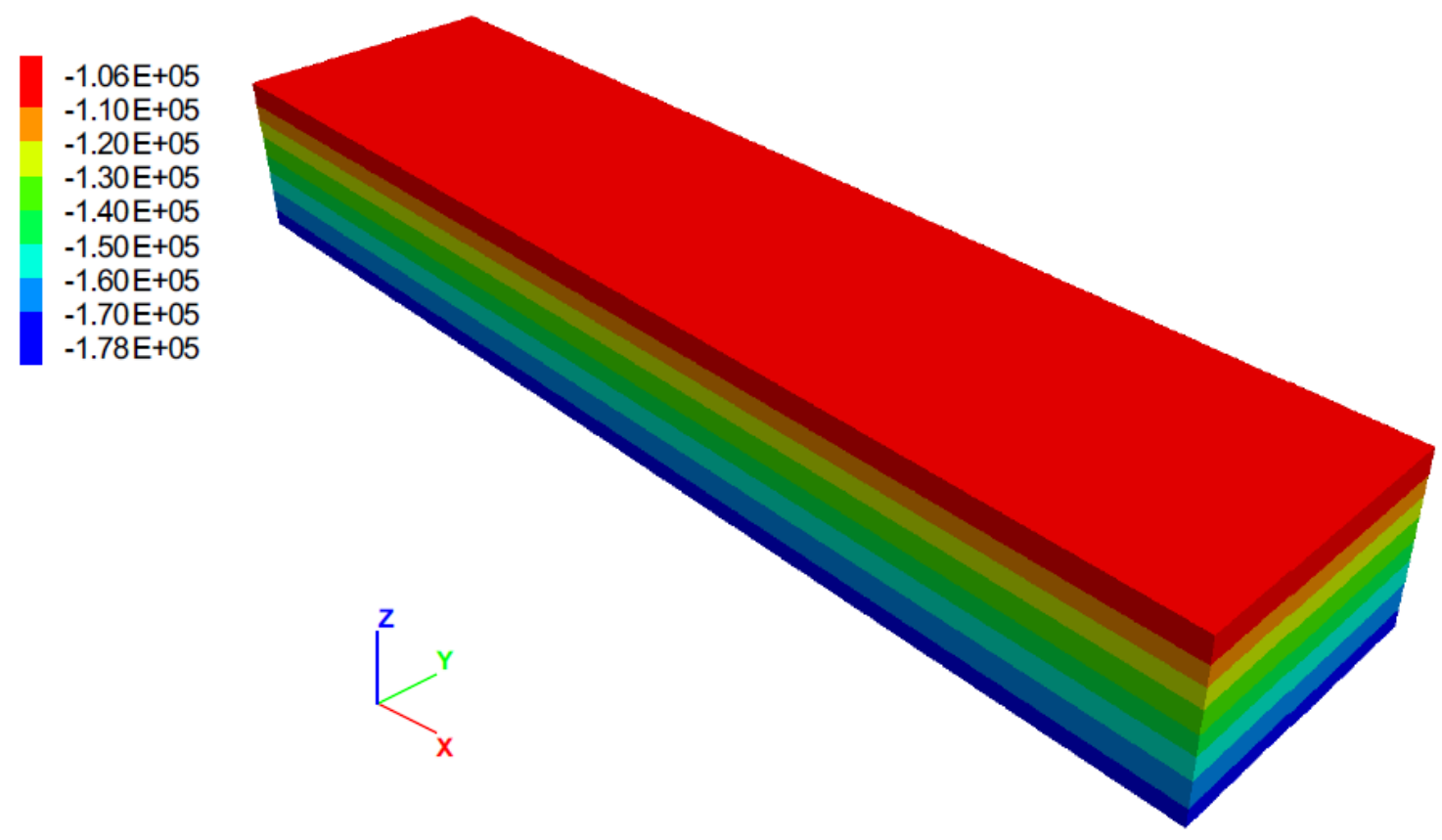

Figure 6.8 Vertical stress contour for the global model (Unit: psf)

Figure 6.9 shows the vertical stress contours in the immediate roof section under two different material property conditions. There is little difference shown in the vertical stress (along $z$ direction) contours. The maximum stress for the deterministic model above the entry and crosscut positions is $5,541.89 \mathrm{psi}$, which is $45 \%$ higher than the random model with spatial variance. 


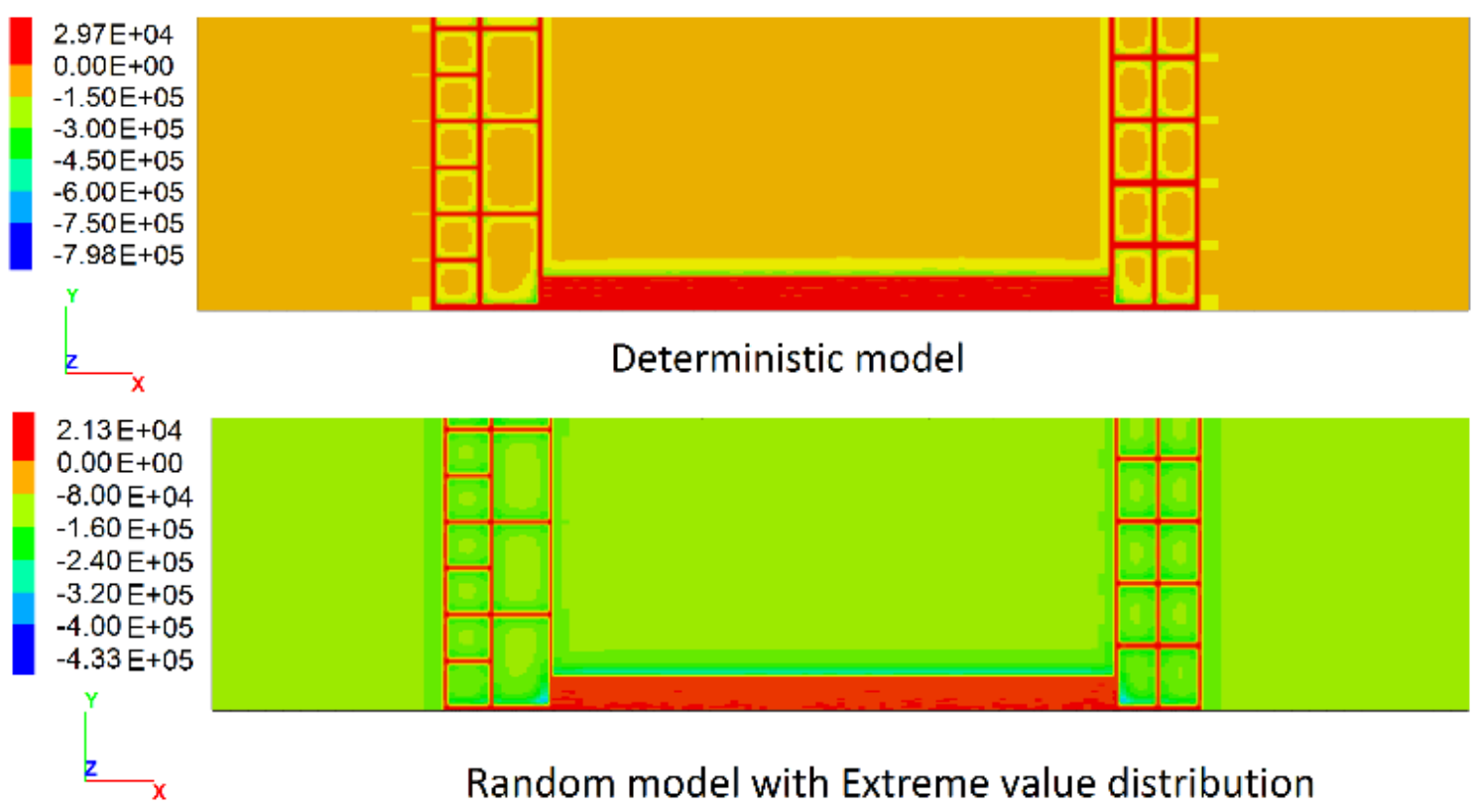

Figure 6.9 Vertical stress contours for the immediate roof section (Unit: psf)

Figure 6.10 shows comparisons of the minimum principal stress for these two different models. The main differences concentrate on the positions above the pillars. The yellow color in the deterministic model and the green color in the random model represent high stress concentration areas. For the deterministic model, only the intersection of the entry and crosscut shows a relatively high-stressed area. However, for the random model, all the areas above the right and left gate roads are green in color. In addition, besides these two locations, the locations close to the rib of the pillars had more areas enter the high stress state for the random model than the deterministic model. 
$-3.84 E+04$ $-5.00 \mathrm{E}+04$ $-7.50 \mathrm{E}+04$ $-1.00 \mathrm{E}+05$ $-1.25 \mathrm{E}+05$ $-1.50 \mathrm{E}+05$ $-1.75 \mathrm{E}+05$ $-2.00 E+05$ $-2.25 \mathrm{E}+05$ $-2.50 E+05$ $-2.75 E+05$ $-3.00 E+05$ $-3.25 E+05$ $-3.50 E+05$ $-3.75 E+05$ $-4.00 \mathrm{E}+05$ $-4.25 \mathrm{E}+05$ $-4.50 \mathrm{E}+05$ $-4.75 \mathrm{E}+05$ $-5.00 E+05$ $-5.01 \mathrm{E}+05$

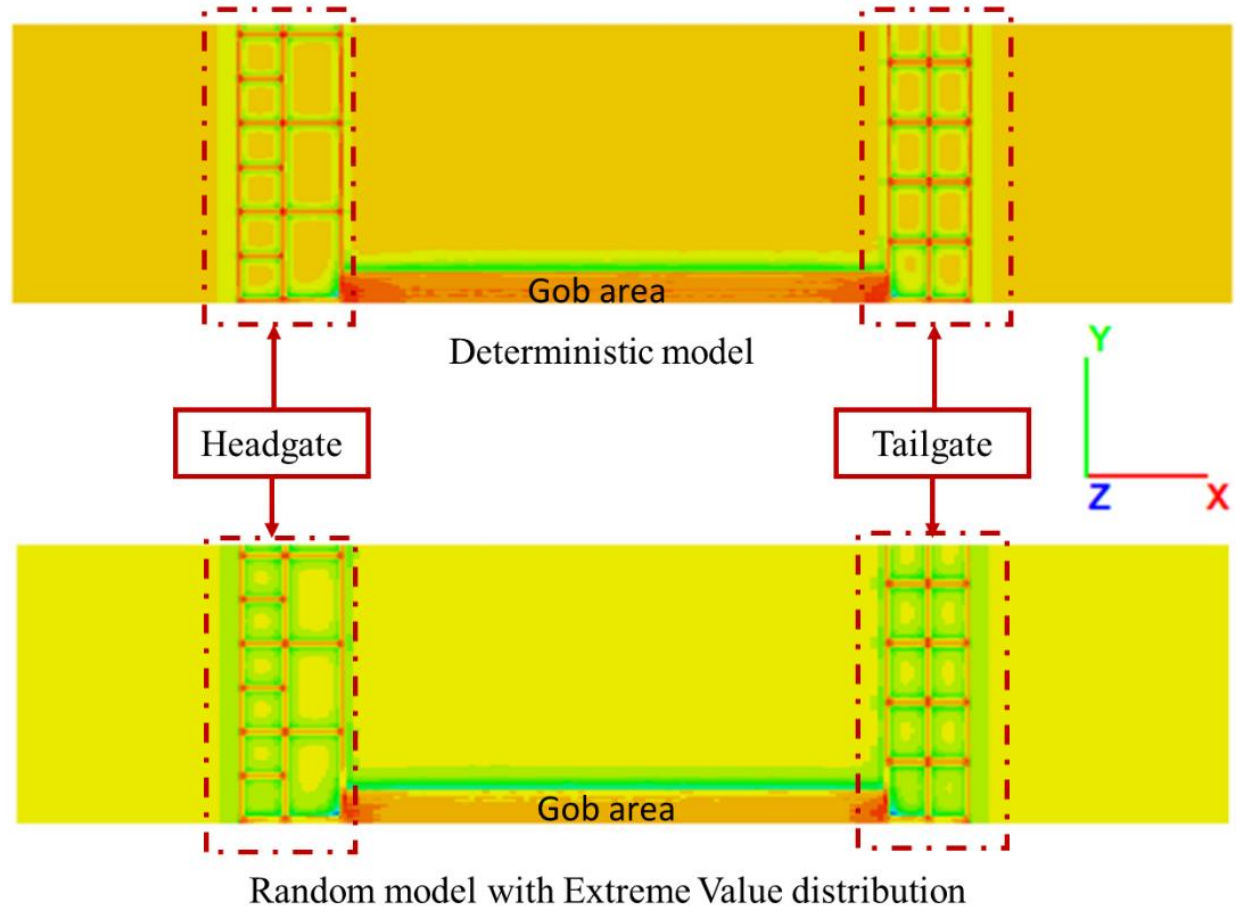

(a) Comparison between headgate
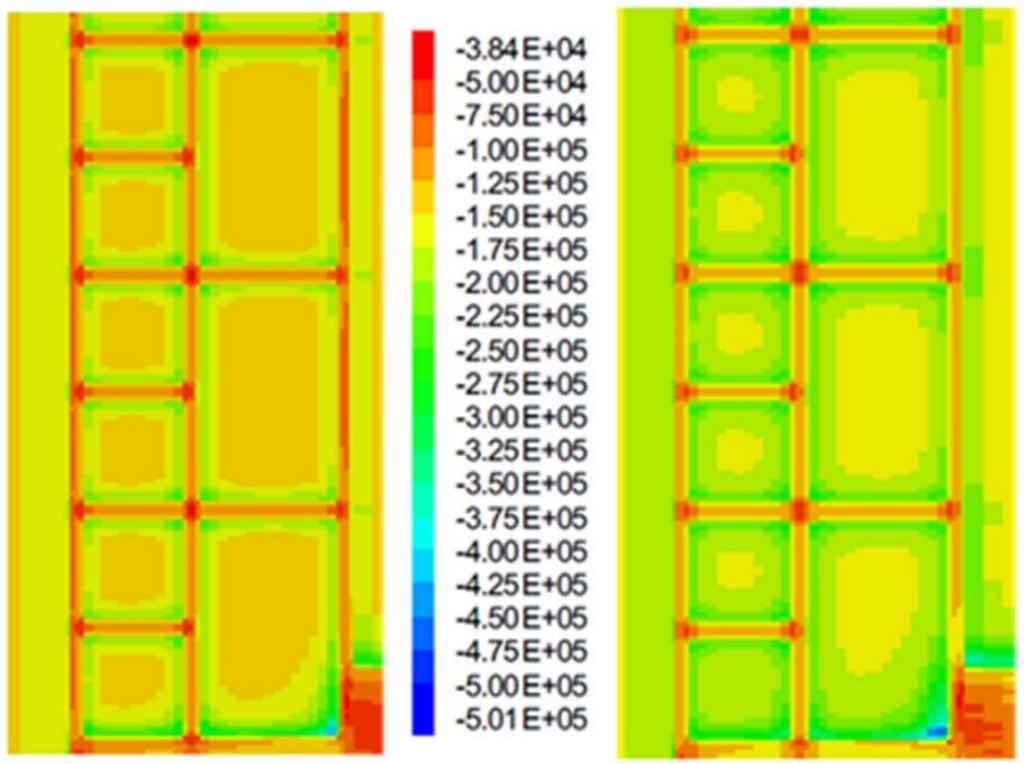

Deterministic model

Random model with Extreme Value distribution 
(b) Comparison between tailgate

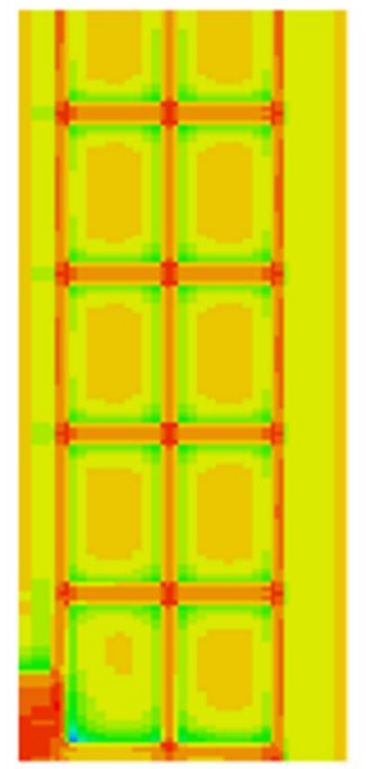

$-3.84 E+04$

$-5.00 E+04$

$-7.50 \mathrm{E}+04$

$-1.00 E+05$

$-1.25 E+05$

$-1.50 E+05$

$-1.75 \mathrm{E}+05$

$-2.00 E+05$

$-2.25 E+05$

$-2.50 E+05$

$-2.75 E+05$

$-3.00 E+05$

$-3.25 E+05$

$-3.50 E+05$

$-3.75 E+05$

$-4.00 E+05$

$-4.25 E+05$

$-4.50 E+05$

$-4.75 E+05$

$-5.00 E+05$

$-5.01 E+05$

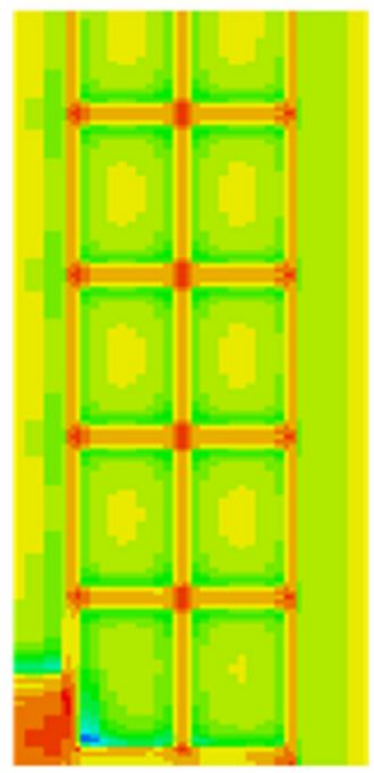

Deterministic model

Random model with Extreme

Value distribution

(c) Comparison between gob area

$-3.84 E+04$

$-5.00 E+04$

$-7.50 \mathrm{E}+04$

$-1.00 E+05$

$-1.25 E+05$

$-1.50 E+05$

$-1.75 E+05$

$-2.00 E+05$

$-2.25 \mathrm{E}+05$

Deterministic model

$-2.50 E+05$

$-2.75 E+05$

$-3.00 E+05$

$-3.25 E+05$

$-3.50 E+05$

$-3.75 E+05$

$-4.00 E+05$

$-4.25 \mathrm{E}+05$

$-4.50 E+05$

$-4.75 E+05$

$-5.00 E+05$

$-5.01 \mathrm{E}+05$

Figure 6.10 Minimum principal stress contours for the immediate roof section (Unit: psf)

Figure 6.11 represents the failure state contours for these two different models. Three different phenomena can be observed: 
Firstly, for the correlated random model, red, red-violet, and green colors show up simultaneously at the locations above the entry, crosscuts, and ribs of the pillars. However, for the deterministic model, only green and red colors appear in the same locations. Moreover, in the tailgate area, some areas in the deterministic model are still in elastic condition, while they already entered shear failure state in the spatial correlated random model. These results imply that, although these two models are set in the same loading conditions and the average values for each parameter are kept the same, the shear failure occurred earlier in the spatial correlated random model due to the only different random characteristic.

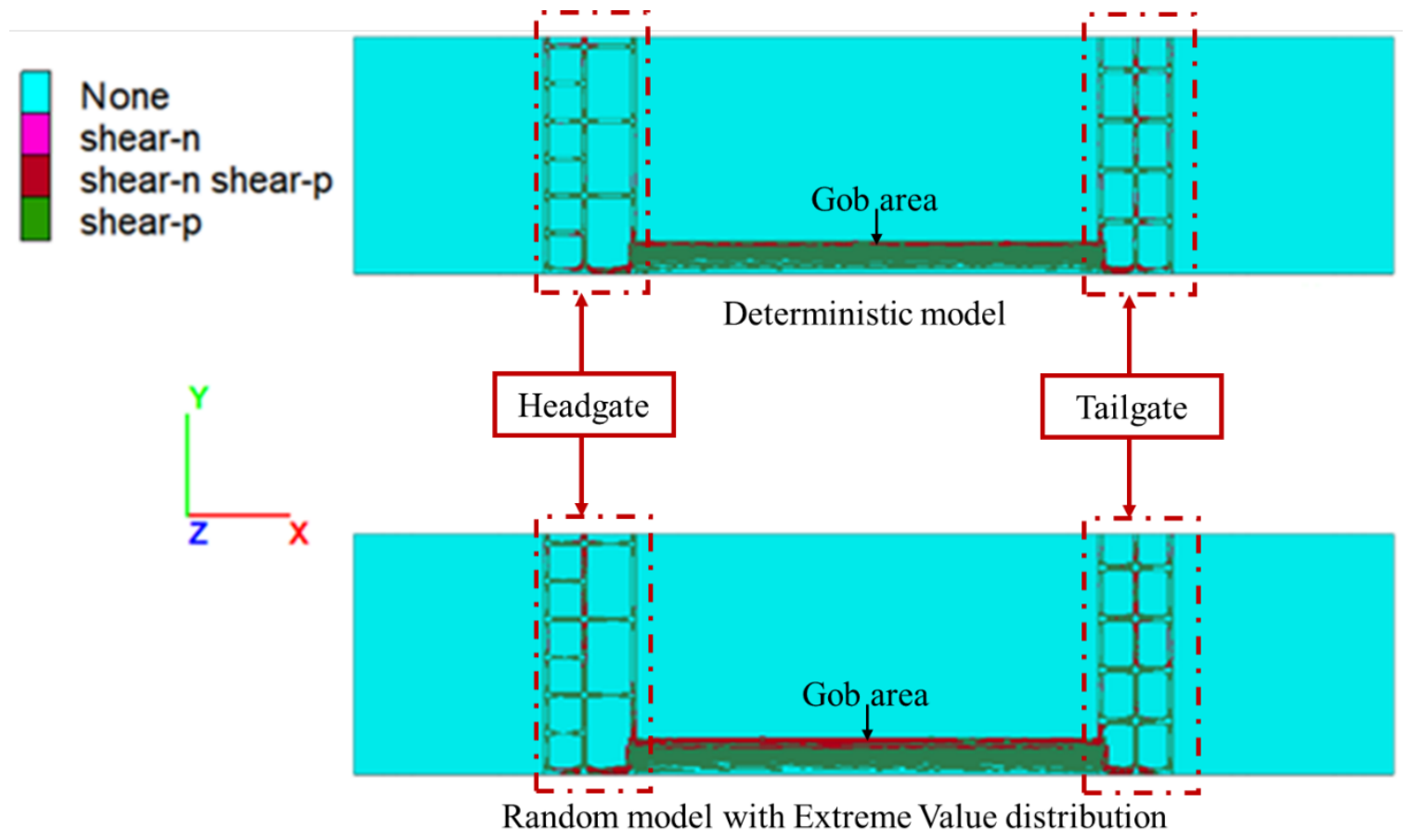


(a) Comparison between headgate

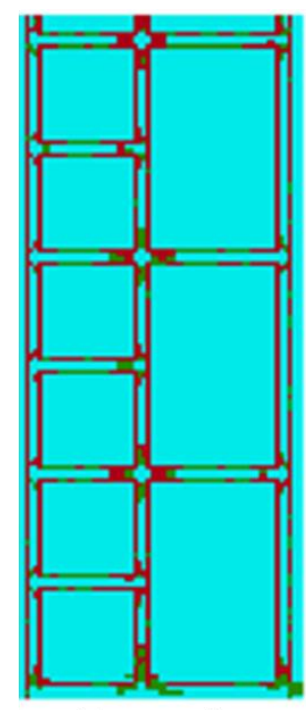

$1^{\text {st }}$ excavation, 20ft excavation along Y-axis

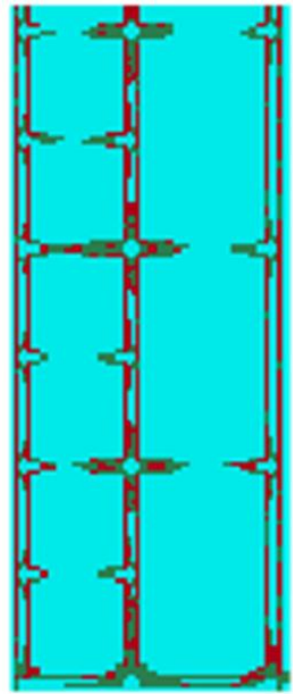

$1^{\text {st }}$ excavation, $20 \mathrm{ft}$ excavation along Y-axis

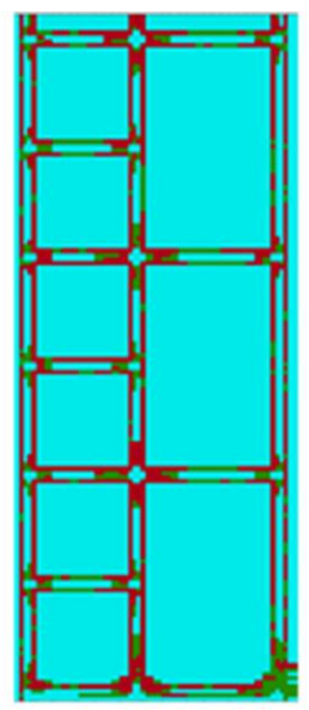

$2^{\text {nd }}$ excavation, 40ft excavation along Y-axis

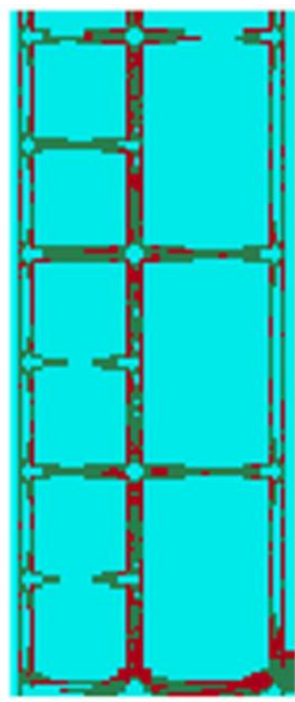

$2^{\text {nd }}$ excavation, $40 \mathrm{ft}$ excavation along Y-axis

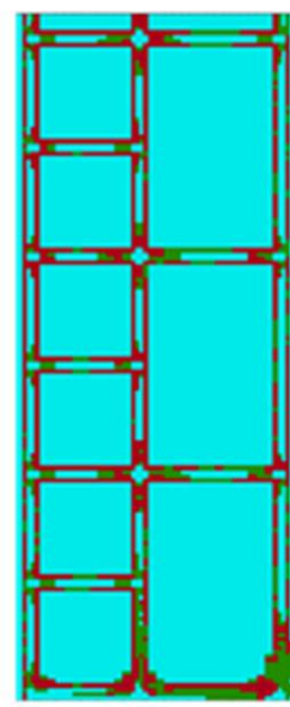

$3^{\text {rd }}$ excavation, $60 \mathrm{ft}$ excavation along Y-axis

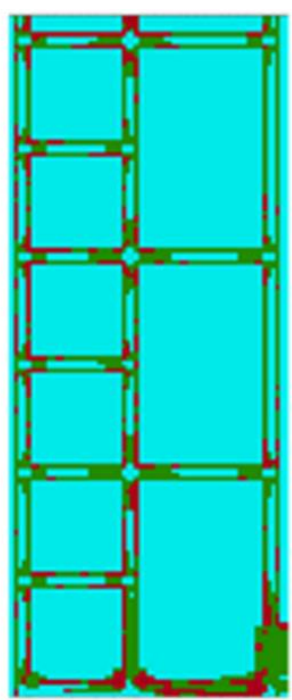

$4^{\text {th }}$ excavation, $80 \mathrm{ft}$ excavation along Y-axis

Deterministic model

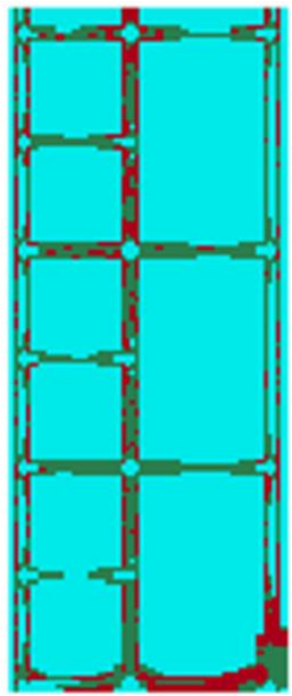

$3^{\text {rd }}$ excavation,

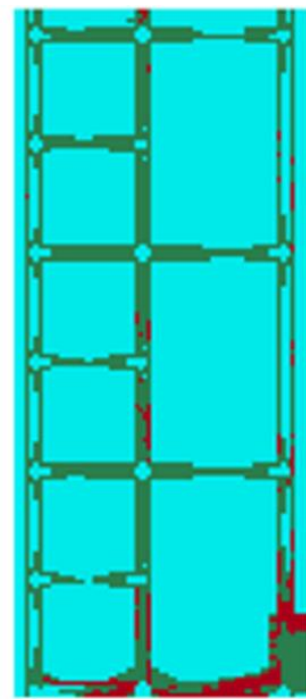

$4^{\text {th }}$ excavation, $60 \mathrm{ft}$ excavation along Y-axis $80 \mathrm{ft}$ excavation along Y-axis

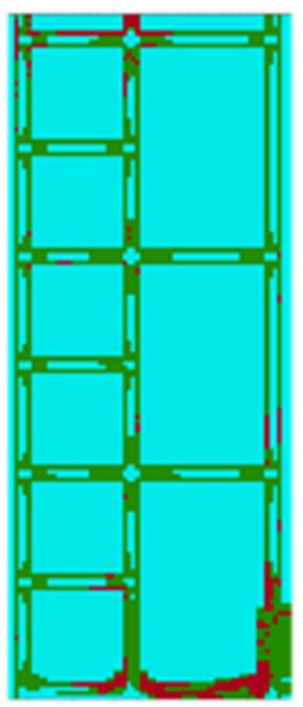

$5^{\text {th }}$ excavation, $100 \mathrm{ft}$ excavation along Y-axis

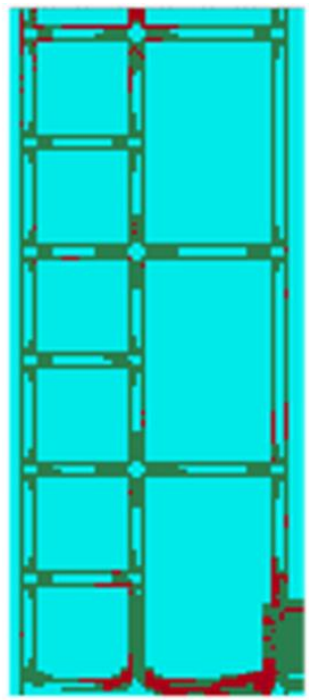

$5^{\text {th }}$ excavation, 100ft excavation along Y-axis

Random model with Extreme Value distribution 
(b) Comparison between tailgate

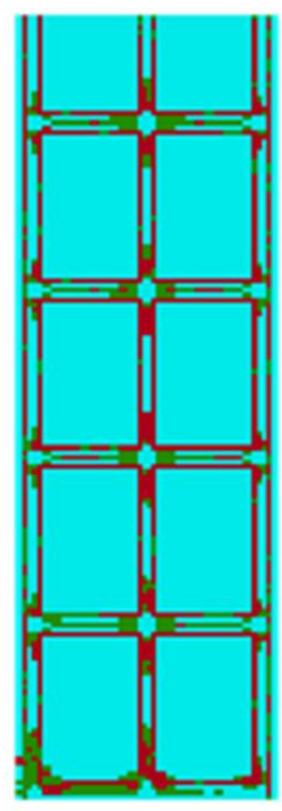

$1^{\text {st }}$ excavation,

$20 \mathrm{ft}$ excavation along Y-axis

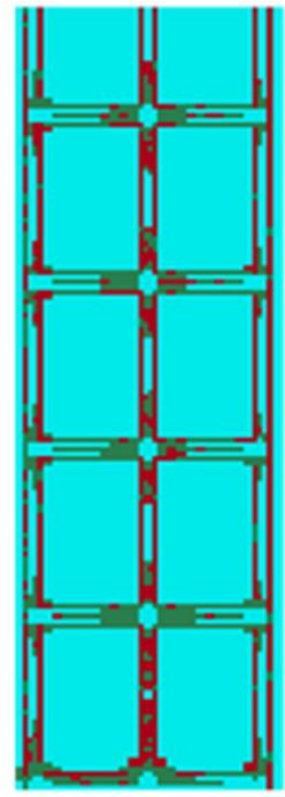

$1^{\text {st }}$ excavation,

$20 f t$ excavation along Y-axis

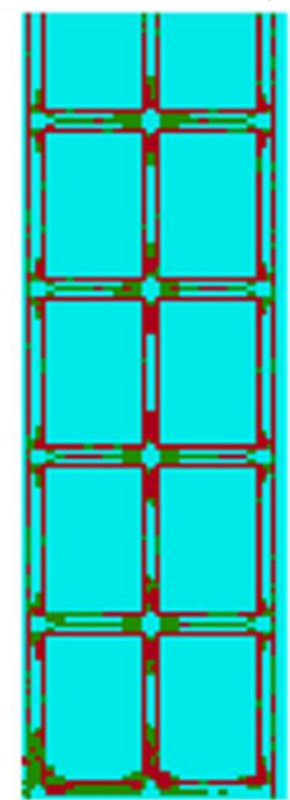

$2^{\text {nd }}$ excavation,

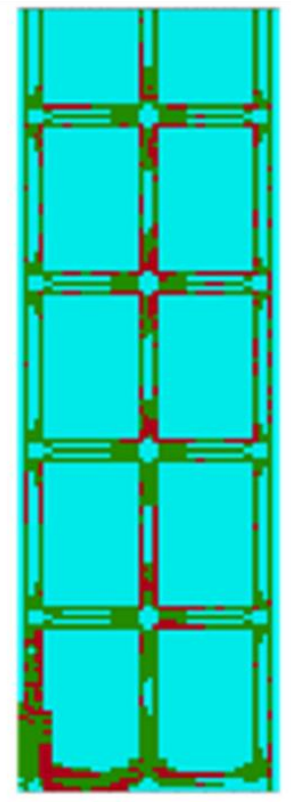

$4^{\text {th }}$ excavation,

Deterministic model

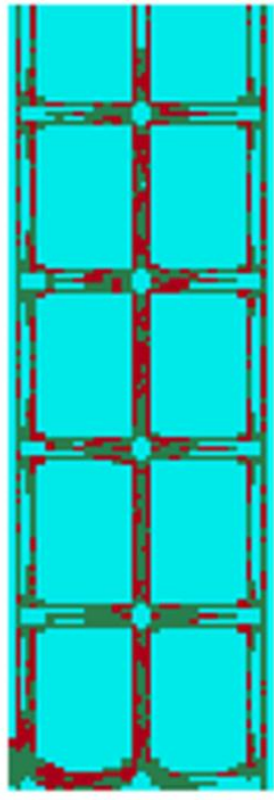

$2^{\text {nd }}$ excavation,

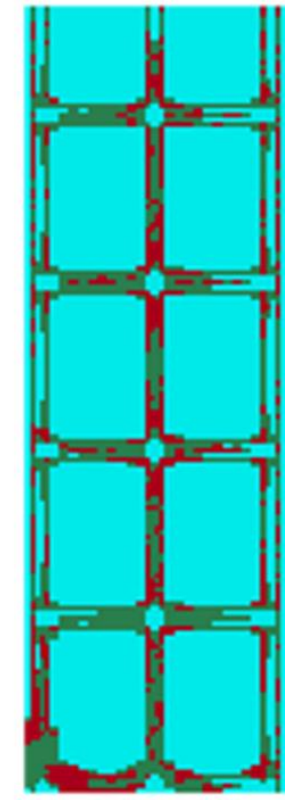

$3^{\text {rd }}$ excavation,

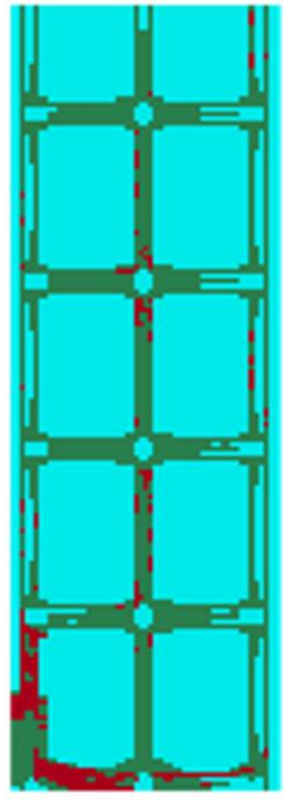

$4^{\text {th }}$ excavation,

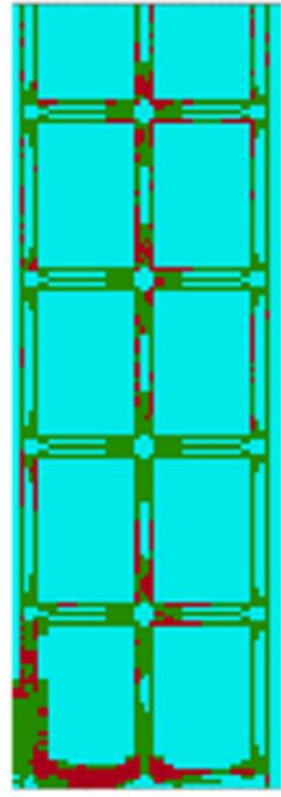

$5^{\text {th }}$ excavation,

$100 \mathrm{ft}$ excavation along Y-axis

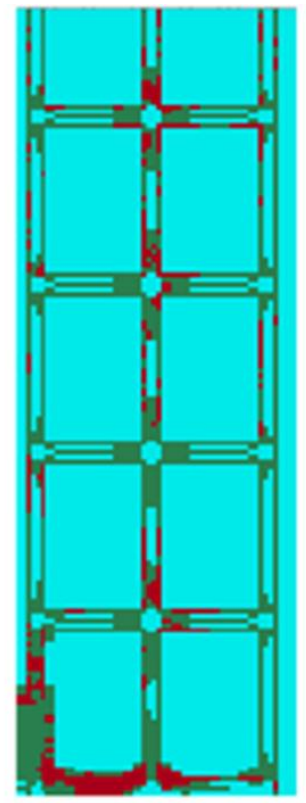

$5^{\text {th }}$ excavation, $100 \mathrm{ft}$ excavation along Y-axis

Random model with Extreme Value distribution 
(c) Comparison between gob area

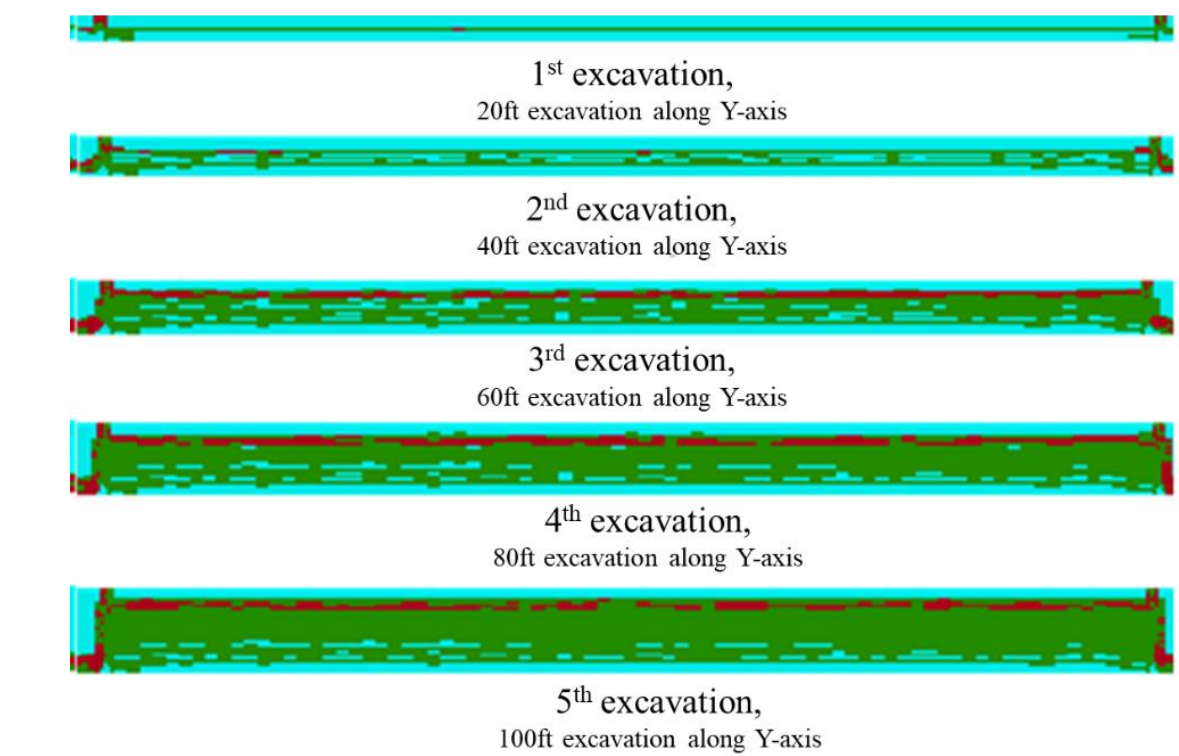

Deterministic model

$1^{\text {st }}$ excavation,

$20 \mathrm{ft}$ excavation along Y-axis

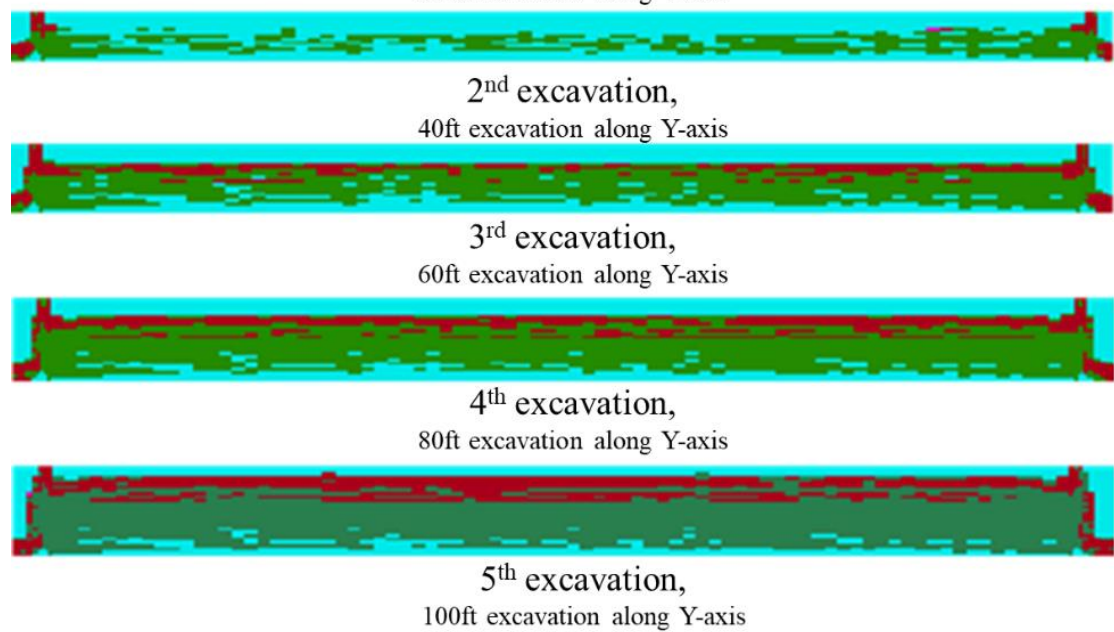

Random model with Extreme Value distribution

Figure 6.11 Failure state contours for the immediate roof section. (Five excavations)

As the cutting sequences occur in the model, both the shear and tension failures are observed in the correlated random model. However, the deterministic model only produced shear failures.

Additionally, for the correlated random model, most of the roof above the gob area entered the shear state. For the intersection between gob area and left gate road, the shear-n state 
occurred, which was different from the same location in the deterministic model. Moreover, more area entered shear-n and shear-p state in the correlated random model than the deterministic model. All these phenomena verify that it is conservative to use the uniform material property to estimate the stability of the mine area.

\subsection{Case three: Gauss distribution used in one pillar scale model}

\subsubsection{Model description}

There are many factors that influence the properties of coal seams, which includes anisotropy, behavior of cracks, pore pressure, rate of loading, time effects, and specimen size and shape. The laboratory result tests on coal can hardly be a proper representation of the in-situ strength data. When researchers perform experiments, all the tests should be performed in an underground environment, not in a laboratory. The influence of the environment is a key point, which cannot be neglected in the rock strength evaluation.

This model included nine rock layers. The layers consisted mostly of four different rock types: silt shale, sandstone, gray clay shale, and coal. All the parameters in this model used the imperial system of units in inches, shown in Table 6.4. 


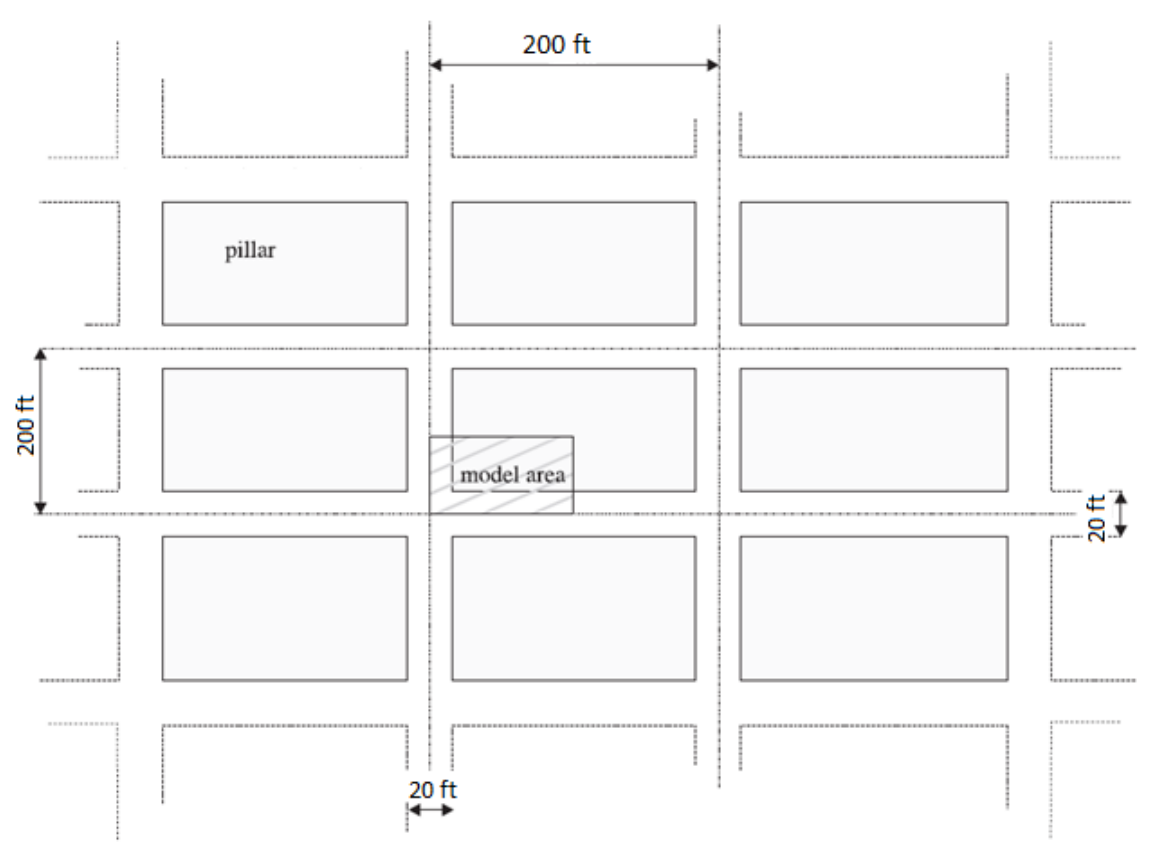

Figure 6.12 Mine layout for the quarter pillar

Table 6.4 Geological material properties for the pillar

\begin{tabular}{|c|c|c|c|c|c|c|}
\hline Rock Type & $\begin{array}{c}\text { Specific } \\
\text { Gravity } \\
\left(\mathbf{l b s} / \mathbf{f t}^{3}\right)\end{array}$ & $\begin{array}{l}\text { Poisson's } \\
\text { ratio }\end{array}$ & $\begin{array}{c}\text { Thickness } \\
\text { (ft.) }\end{array}$ & $z$ coordinates & $\begin{array}{c}\text { Bulk } \\
\text { Modulus } \\
(\mathbf{p s i})\end{array}$ & $\begin{array}{c}\text { Shear } \\
\text { Modulus } \\
(\text { psi) }\end{array}$ \\
\hline Silt shale & 160 & 0.2 & 5 & 60 & $1.66 \times 10^{6}$ & $1.24 \times 10^{6}$ \\
\hline Sandstone & 167 & 0.15 & 52 & 684 & $1.41 \times 10^{6}$ & $1.28 \times 10^{6}$ \\
\hline Silt shale & 170 & 0.2 & 8 & 780 & $1.66 \times 10^{6}$ & $1.24 \times 10^{6}$ \\
\hline $\begin{array}{l}\text { Gray clay } \\
\text { shale }\end{array}$ & 166 & 0.27 & 2 & 804 & $4.93 \times 10^{5}$ & $2.68 \times 10^{5}$ \\
\hline Freeport seam & 88 & 0.34 & 6.5 & 882 & $3.75 \times 10^{5}$ & $1.34 \times 10^{5}$ \\
\hline $\begin{array}{l}\text { Gray clay } \\
\text { shale }\end{array}$ & 166 & 0.27 & 5 & 942 & $4.93 \times 10^{5}$ & $2.68 \times 10^{5}$ \\
\hline Silt shale & 164 & 0.27 & 5 & 1,002 & $1.66 \times 10^{6}$ & $1.24 \times 10^{6}$ \\
\hline Silt shale & 160 & 0.2 & 50 & 1,602 & $1.66 \times 10^{6}$ & $1.24 \times 10^{6}$ \\
\hline Sandstone & 167 & 0.15 & 50 & 2,202 & $1.41 \times 10^{6}$ & $1.28 \times 10^{6}$ \\
\hline Rock Type & $\begin{array}{c}\text { Tension } \\
\text { Modulus } \\
\quad(p s i)\end{array}$ & $\begin{array}{l}\text { Cohesion } \\
\quad(\text { psi) }\end{array}$ & Friction $\left({ }^{\circ}\right)$ & $\begin{array}{l}\text { Vertical stress } \\
\text { (psi) }\end{array}$ & $\begin{array}{l}\text { Horizontal } \\
\text { stress (psi) }\end{array}$ & $\begin{array}{c}\text { Horizonta } \\
\text { gradient } \\
\left.\left(\mathrm{lbs}_{\mathrm{ibn}}\right)^{3}\right)\end{array}$ \\
\hline Silt shale & 193 & 523 & 33 & $1,085.42$ & 271.35 & 0.02 \\
\hline Sandstone & 325 & 846 & 35 & $1,145.72$ & 202.19 & 0.02 \\
\hline
\end{tabular}




\begin{tabular}{|c|c|c|c|c|c|c|}
\hline Silt shale & 193 & 523 & 33 & $1,155.17$ & 288.79 & 0.02 \\
\hline $\begin{array}{c}\text { Gray clay } \\
\text { shale }\end{array}$ & 120 & 354 & 29 & $1,157.47$ & 428.11 & 0.04 \\
\hline $\begin{array}{c}\text { Freeport seam } \\
\text { Gray clay } \\
\text { shale }\end{array}$ & 120 & 354 & 29 & 1167.21 & 431.71 & 0.04 \\
\hline Silt shale & 193 & 523 & 33 & $1,172.90$ & 433.81 & 0.04 \\
\hline Silt shale & 193 & 523 & 33 & $1,228.46$ & 307.11 & 0.02 \\
\hline Sandstone & 325 & 846 & 35 & $1,286.44$ & 227.02 & 0.02 \\
\hline
\end{tabular}

This study applied the roller boundary condition for the lateral faces, and the fixed boundary condition for the bottom face. The vertical stress applied on the first layer "silt shale" was equal to the pressure, caused by the gravity of the overburden layers. The relationship between vertical stress $\sigma_{v}$ and horizontal stress $\sigma_{h}$ is expressed as:

$$
\sigma_{h}=\frac{v}{(1-v)} \times \sigma_{v}
$$

where $v$ is the Poisson's ratio.

The immediate roof and coal seam parts were randomly material distributed layers, which satisfied Gauss distribution. The other layers were defined as uniform material stratum, as shown in Figure 6.13. 


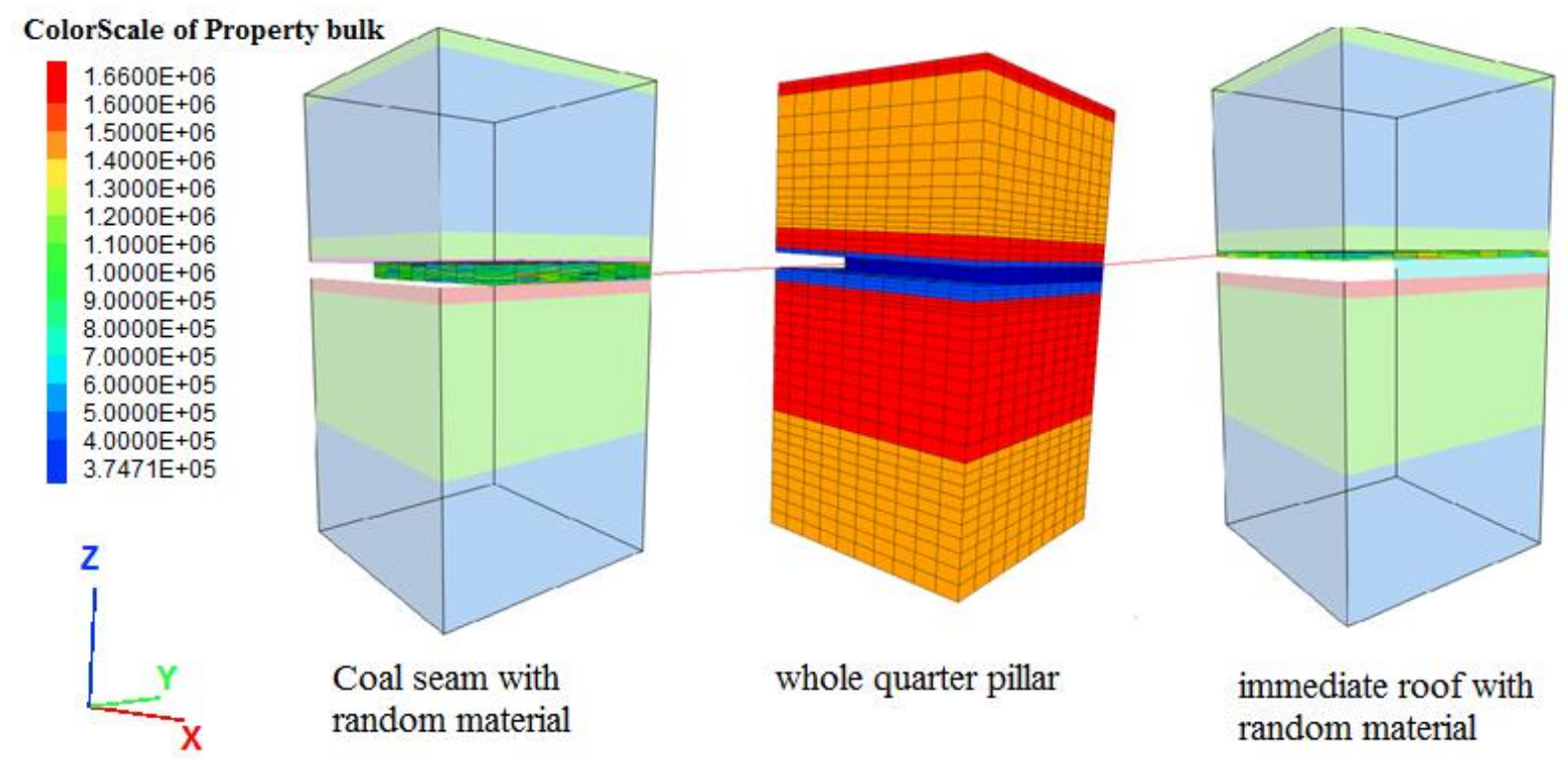

Figure 6.13 Quarter pillar with stochastic material definition (Unit: psi)

\subsubsection{Result discussions}

The purpose of this model is to summarize the stresses around a specific rock specimen, extract groups of specimens out of the pillar in random locations and compare the peak strength versus the dimensions of specimens based on stochastic theory. After the regression analysis, the peak strength will approach an asymptotic value as the size of the specimen increases. As such, the strength for both rock specimen and rockmass could be estimated in a more realistic situation. 


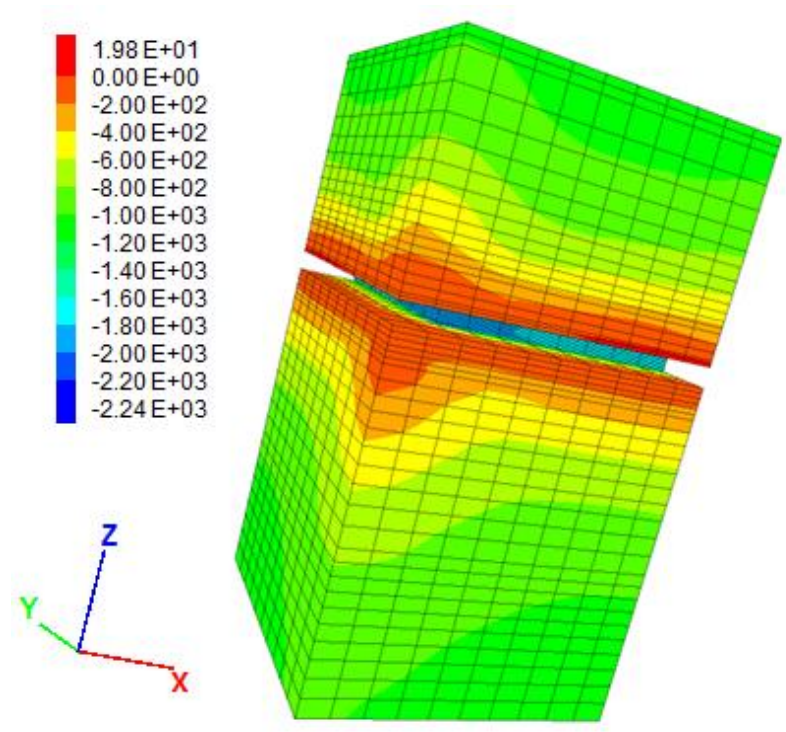

(a) Minimum principal stress (Unit: psi)

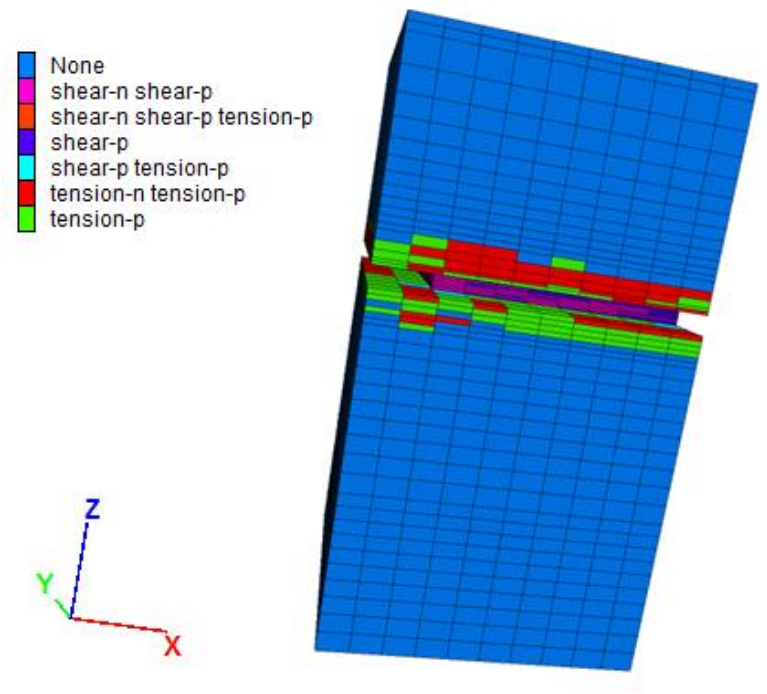

(b) Yield state distribution

Figure 6.14 Result contours for the quarter pillar

Here, the advantage of considering rock specimens in this method is that the influence of the environment can be eliminated as they move the specimens from site to the laboratory. The specimens always sit in their original field positions and take pressure as in their initial situations.

From Figure 6.14, the minimum principal stress contour shows that tension area occurred in both roof and floor, especially in the corner of the intersection. Rock has much lower tension bearing capacity than compression, which means rock material is very sensitive to tension force. Therefore, in this case, failure is probable to occur in roof and floor parts. This inference also is true in the yield state contour; while some tension failure states show in both roof and floor, more tension failure happened in the roof than in the floor. The mean values of the materials for the immediate roof and floor are the same, the only difference being that the material for the roof part is in random condition, while the material for floor is in uniform condition. The reason for the failure happening earlier in the immediate roof than the floor is due to the peak strength value of traditional determination calculation being higher, which is conservative to the future safety factor calculation. 


\subsection{Conclusions}

At rockmass level, the material properties of rock are neither deterministic nor completely random. The random model with spatial correlation is much closer to the case in the field. The focus of this research is to demonstrate that heterogeneity and discontinuity significantly affect rock strength and failure process in the field-scaled model. Conclusions are summarized as follows:

Based on the improved the conventional Type III Extreme Value distribution by adding the spatial correlation factor, the spatial correlation revealed both the correlation and continuity that exists inherently in rock. The numerical simulation results show that the spatial correlated random model can estimate the rock strength in a relatively more realistic way.

More high stress concentration area occurs around the rib of the pillars in the random model with the spatial variance factor than the completely deterministic model.

As the cutting sequence progressed, the tensile failure occurred in locations above the gob area and gate roads.

Based on the vertical stress of the deterministic model being around $45 \%$ higher than the spatially correlated random model observed in this research, we can conclude that the deterministic method used to estimate the stability of roof parts in real longwall mine is conservative.

Based on the discussions summarized in the earlier sections, the research showed that the traditionally deterministic method is conservative to some extent. Exclusively for roof stability analysis, the spatial variance is a significant factor, which cannot be ignored. 


\section{CHAPTER 7 CONCLUSIONS}

The primary objective of this dissertation was to use the revised probabilistic method to demonstrate the heterogeneity characteristic of rock from the perspective of material properties, as well as to observe the influences of spatial variance on the failure mechanism for both rock specimens in laboratory and rockmass in the real field site.

The primary methodology was the Type III Extreme Value distribution of the smallest value by adding the spatial correlation factor. The entire process of this research can be summarized through the following steps:

First, this study made comparisons between the deterministic and completely random models based on the laboratory tests. The main goal of this step was to show the importance of considering the randomness factor of rocks.

Second, this research considered one spatial correlated random model to investigate the influence of rock heterogeneity on the rock strength and failure propagation. Random field database was created with specific spatial correlation for each physical-mechanical property using the rough laboratory data and Extreme Value stochastic model in MATLAB. They also added two scale-measured parameters to define the correlation length, which could control the spatially correlated random data.

Third, in order to verify the importance of four parameters, friction, cohesion, and correlation length along horizontal and vertical axes respectively, this research generated 152 random sample data. Stress for each specimen at different loading steps and different locations. The aim 
of this process was to find out how the inherent material parameters affect the internal stress for intact rocks.

Fourth, in order to have confidence in the model results, some form of calibration of the model with the Hoek-Cell triaxial laboratory test was necessary. The real spatial variance was established distribution through experiments. Based on the mechanical parameters obtained from the laboratory, the proposed method reproduced the material distribution of the intact rock block. The two results above match with each other, which verified that the proposed stochastic method is appropriate for this research.

Finally, two three-dimensional longwall mine models were analyzed using the proposed method. The influence of random field data on entry roofs in the longwall mining system is expected. This research created the realistic random field database based on the Extreme Value stochastic model, adding two scale-measured parameters from both horizontal and vertical directions to control the spatial correlation length. This research considered a few cutting sequences in this model, which could be used to observe if the spatial variance had any significant influence over the roof behavior.

This dissertation draws the following conclusions from the studies performed throughout this process:

Use of the deterministic method results in a conservative estimation of rock strength. Use of the deterministic method overestimates the rock strength by $50 \%$. The peak strength decreased as the element number of random models increased.

For the deterministic model, the failure always behaves like a double pyramid type of failure. The yield zones are relatively concentrated. For the stochastic models in this 
research, both the macro-cracks and micro-cracks both can be monitored. Each microcrack will cause stress redistribution and propagate, which in turn will cause a severe failure. As such, the failure pattern for rock specimen with stochastic analysis is less predictable. The residual strength for the intact rock varies extensively with the grid number.

Based on one type stochastic model, this research improved the conventional Type III Extreme Value distribution by adding the spatial correlation factor. The spatial correlation revealed both the correlation and continuity that exists inherently in rock. The numerical simulation results show that the spatial correlated random model can estimate the rock strength in a relatively more realistic way.

For the spatial correlated random model, the small cracks normally initiate at relatively weak zones. As the spatial dependence increases, the failure state of the rock specimen changes from random to a relatively stable state.

Friction angle is the primary factor causing damage of the rock sample. After initial failure happens, the spatial correlated friction angle will combine with spatial correlated cohesion value to influence the following failure propagation.

More high stress concentration area occurred around the rib of the pillars in the random model with the spatial variance factor than in the completely deterministic model for the longwall mine.

For the longwall mine, as the cutting sequence progressed, the tensile failure occurred in locations above the gob area and gate roads.

Based on the vertical stress of the deterministic model being around $45 \%$ higher than the spatially correlated random model observed in this research, we concluded that the 
deterministic method used to estimate the stability of roof part in real longwall mine is conservative.

\section{RECOMMENDATIONS FOR FUTURE RESEARCH}

The stochastic model with spatial correlation factor can represent the behavior of rock material realistically, especially in the post-failure region in this research. The revised Extreme Value distribution applied in the triaxial model indicates that the results between laboratory test and numerical simulation are consistent with each other. Therefore, the new stochastic model with the addition of the spatial correlation factor model may be a better option to represent the real mechanical behavior of rockmass. However, this research only considered two scaled parameters for the correlation factor. In the future, studies could also incorporate the third scaled parameter to simulate the random field characteristic of rockmass. In addition, this research only considered the calibration work for the laboratory scaled model. More field observations or measurements will further refine and validate the model 


\section{REFERENCES}

Amusin, B. Z., 1979. Mechanical characteristics of rock in place in analytical calculations on rock pressure phenomena in workings. Soviet Mining 15.6: 551-556.

Bagtzoglou, Amvrossios C. and Baca, R. G., 1993. Probabilistic calculations of groundwater travel time in heterogeneous three-dimensional porous media. MRS Online Proceedings Library Archive 333.

Barton, N., Lien, R., and Lunde, J., 1974. "Engineering classification of rockmasses for the design of tunnel support." Rock mechanics 6.4: 189-236.

Bažant, Zdeněk P., Mazen R. Tabbara, Mohammad T. Kazemi, and Gilles Pijaudier-Cabot.,1990. Random particle model for fracture of aggregate or fiber composites. Journal of engineering mechanics 116, no. 8: 1686-1705.

Beacher, G. B., and Ingra, T. S., 1981. "Stochastic FEM in settlement predictions." Journal of Geotechnical and Geoenvironmental Engineering 107.ASCE 16179.

Bieniawski, Z. T., 1993. Classification of rockmasses for engineering: the RMR system and future trends. Rock Testing and Site Characterization. Pergamon: 553-573.

Bieniawski, Z. T., 1978. Determining rockmass deformability: experience from case histories. International journal of rock mechanics and mining sciences $\&$ geomechanics abstracts. Vol. 15. No. 5. Pergamon.

Bieniawski, Z. T., 1968. The effect of specimen size on compressive strength of coal. International Journal of Rock Mechanics and Mining Sciences \& Geomechanics Abstracts. Vol. 5. No. 4. Pergamon. 
Brady, B. G. H., \& Brown, E. T., 1993. Rock mechanics for underground mining. London: Chapman \& Hall.

Brown, E. T., 1974. Fracture of rock under uniform biaxial compression. Proc. of the 3th Int. Cong. of Rock Mech. Vol. 2.

Chaplin, W. S., 1880. The relation between the tensile strengths of long and short bars. Van Nostrand's Engineering Magazine 23.144: 441.

Chen, Q., Seifried, A., Andrade, J. E., \& Baker, J. W., 2012. Characterization of random fields and their impact on the mechanics of geosystems at multiple scales. International Journal for Numerical and Analytical Methods in Geomechanics, 36(2), 140-165..

Chun'an, T., 1997. Numerical simulation of progressive rock failure and associated seismicity. International Journal Of Rock Mechanics And Mining Sciences \& Geomechanics Abstracts. Vol. 34. No. 2 Elsevier Science.

Deng, J., Yue, Z. Q., Tham, L. G., \& Zhu, H. H., 2003. Pillar design by combining finite element methods, neural networks and reliability: a case study of the Feng Huangshan copper mine, China. International Journal of Rock Mechanics and Mining Sciences, 40(4), 585599.

Einstein, H. H., and Baecher, G. B., 1983. Probabilistic and statistical methods in engineering geology. Rock mechanics and rock engineering 16.1: 39-72.

Fang, Z., and Harrison, J. P., 2002. Development of a local degradation approach to the modelling of brittle fracture in heterogeneous rocks. International Journal of Rock Mechanics and Mining Sciences 39(4): 443-457. 
Gao, D., Mishra, B., and Xue, Y., 2020. Numerical Simulation of Laboratory Strength Tests Using a Stochastic Approach. Mining, Metallurgy \& Exploration 37.2: 709-716.

Gao, H., Shen, G., and Li, B., 1987. The effect of mining pressure and hydraulic pressure on water inrush in floor. Journal of Coal Geology and Exploration, No. 3: 50-60.

Griffith, A. A., 1921. VI. The phenomena of rupture and flow in solids. Philosophical transactions of the royal society of london. Series A, containing papers of a mathematical or physical character 221.582-593: 163-198.

Griffiths, D. V., Fendon, G. A., and Lemons, C. B., 2007. The random finite element method (RFEM) in mine pillar stability analysis. Probabilistic methods in geotechnical engineering. Springer, Vienna: 271-294.

Gu, D. Z., 1979. Fundamentals of rock-mass engineering geomechanics. Beijing: China Science Press: pp75-87.

Gumbel, E. J., 1958. Statistical theory of floods and droughts. Journal of the Institution of Water Enigneers and Scientists 12: 157-184.

Gureev, A. M., and Voronkov, O. K., 1974. Geostructural, Geophysical and geomechanical schemes of rockmasses. Proc., 3rd Congress of 1SRM: 46-51.

Haldar, A., and Mahadevan, S., 2000. Probability, reliability, and statistical methods in engineering design. John Wiley.

Hoek, E., and Brown, E. T., 1980. Underground Excavations in Rock. Boca Raton, FL: CRC Press: p.527.

Hoek, E., 1994. Strength of rock and rockmasses, ISRM News Journal, 2, 4-16.: pp 4-16. 
Hoek, E., Kaiser, P. K., and Bawden, W. F., 1995. Support of underground excavations in hard rock. AA Balkema, Rotterdam.

Hoek, E., and Brown, E. T., 1997. "Practical estimates of rockmass strength." International journal of rock mechanics and mining sciences 34.8: 1165-1186.

Hubler, M. H., Gelb, J., and Ulm, F. J., 2017. "Microtexture analysis of gas shale by XRM imaging." Journal of Nanomechanics and Micromechanics 7.3: 04017005.

Itasca, FLAC, 2017. https://www.itascacg.com/software/flac. Itasca Consulting Group, Inc. Minnesota, USA.

Jade, S., and Sitharam, T. G., 2003. Characterization of strength and deformation of jointed rockmass based on statistical analysis. International journal of Geomechanics 3.1: 43-54.

Kim, K., and Gao, H., 1995. Probabilistic approaches to estimating variation in the mechanical properties of rockmasses. International Journal of Rock Mechanics and Mining Sciences \& Geomechanics Abstracts 32(2): 111-120.

Kim, K., and Gao, H., 1995. Probabilistic approaches to estimating variation in the mechanical properties of rockmasses. International journal of rock mechanics and mining sciences \& geomechanics abstracts. Vol. 32. No. 2. Pergamon.

La Pointe, P. R., and Hudson, J. A., 1985. Characterization and interpretation of rockmass joint patterns. Vol. 199. Geological Society of America.

Ledvina, C. T., 1991. Geostatistical inference and exploration of coal mine roof strata. Diss. Northwestern University.

Mark, C., 1987. Analysis of Longwall Pillar Stability. PhD Thesis. Pennsylvania State University. 
Matheron, G., 1971. The theory of regionalised variables and its applications. Les Cahiers du Centre de Morphologie Mathématique 5: 212.

Mingli, H., Chunan, T., and Wancheng, Z., 2000. Numerical simulation on failure process of rock [J]. Chinese Journal of Rock Mechanics and Engineering 19(4): 468-471.

Peng, S. S., and Dutta, D., 1992. Evaluation of various pillar design methods: a case study. Prodeedings of the Workshop on Coal Pillar Mechanics and Design, 33rd US Symposium on on Rock Mechanics.

Potvin, Y., Dight, P. M., and Wesseloo, J., 2012. Some pitfalls and misuses of rockmass classification systems for mine design. Journal of the Southern African Institute of Mining and Metallurgy 112.8: 01-06.

Priest, S. D., and Hudson, J. A., 1976. Discontinuity spacings in rock. International Journal of Rock Mechanics and Mining Sciences \& Geomechanics Abstracts. Vol. 13. No. 5. Pergamon.

Recio-Gordo, D., and Jimenez, R., 2012. A probabilistic extension to the empirical ALPS and ARMPS systems for coal pillar design. International journal of rock mechanics and mining sciences (1997) 52: 181-187.

Salamon, M. D. G., and Munro, A. H., 1967. A study of the strength of coal pillars. Journal of the Southern African Institute of Mining and Metallurgy 68.2: 55-67.

Scovazzo, V. A., 1992. A practitioner's approach to pillar design. Prodeedings of the Workshop on Coal Pillar Mechanics and Design, 33rd US Symposium on on Rock Mechanics. 
Soltani, A., 2015. Analysis of the ARMPS database using FLAC3d; a pillar stability comparison for room and pillar coal mines during development.

Vanmarcke, E. H., 1977. Probabilistic modeling of soil profiles. Journal of the geotechnical engineering division 103.11: 1227-1246.

Villaescusa, E., and Brown, E. T., 1990. Characterizing joint spatial correlation using geostatistical methods. International symposium on rock joints.

Weibull, W., 1952. A survey of statistical effects in the field of material failure. Appl. Mech. Rev 5.11: 449-451.

Weibull, W., 1939. A statistical theory of the strength of materials. Ingeniors Vetenskaps Akademien.

Wilding, L. P., and Drees, L. R., 1978. Spatial Variability: A Pedologist's Viewpoint1. Diversity of Soils in the Tropics: ASA Special Publication Number 34 34: 1-12.

X., Yu., 1983. Stability analysis of underground openings. Beijing: China Coal Industry Press.

Yamaguchi, U., 1970. The number of test-pieces required to determine the strength of rock. International Journal of Rock Mechanics and Mining Sciences \& Geomechanics Abstracts. Vol. 7. No. 2. Pergamon.

Yeglalp, T. M., and Mahtab, M. A., 1983. A proposed model for statistical representation of mechanical properties of rock. The 24th US Symposium on Rock Mechanics (USRMS). American Rock Mechanics Association. 
Yegulalp, T. M., and Kim, K., 1994. Statistical assessment of scale effect on rock properties using the theory of extremes. Transactions-Society of Mining Engineers of AIME: 18341834.

Yegulalp, Tuncel M., and Malcolm T. Wane., 1968. Application of extreme valus statistics to test data. Trans. Soc. Min. Eng., AIME 241: 372-376.

Yu, Y. F., and G. R. Mostyn., 1993. Spatial correlation of rock joints. Conference on probabilistic methods in geotechnical engineering.

Zhang, W., and S. Valliappan., 1990. Analysis of random anisotropic damage mechanics problems of rockmass statistical estimation. Rock Mechanics and Rock Engineering 23.4: 241-259. 


\section{VITA}

Danqing Gao is originally from Nantong city, Jiangsu province, P.R. China. She joined the Mining Engineering Department at WVU in January 2015. Prior to joining WVU, Gao has three and half years civil test engineer working experience in China. Gao earned her B.S. in Engineering Mechanics from China Agricultural University, and M.S. in Engineering Mechanics from Beijing University of Science and Technology, respectively.

Danqing Gao 